\title{
Fast Plasma Investigation for Magnetospheric Multiscale
}

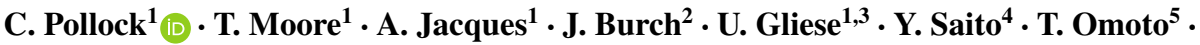
L. Avanov ${ }^{1,6}$ - A. Barrie ${ }^{1,7}$ - V. Coffey ${ }^{8}$ J. Dorelli ${ }^{1}$ - D. Gershman ${ }^{1,6,9}$ - B. Giles ${ }^{1}$.

T. Rosnack $^{1,10}$ - C. Salo ${ }^{1,11}$ - S. Yokota ${ }^{4}$ M. Adrian ${ }^{1}$ - C. Aoustin ${ }^{12}$ - C. Auletti ${ }^{1,13,14}$.

S. Aung ${ }^{1,10} \cdot$ V. Bigio ${ }^{1}$ - N. Cao ${ }^{1,15} \cdot$ M. Chandler $^{8}$ - D. Chornay ${ }^{1,6}$ - K. Christian ${ }^{1,16}$.

G. Clark ${ }^{1,17,18}$ - G. Collinson ${ }^{1,17}$ - T. Corris ${ }^{1,3}$ - A. De Los Santos ${ }^{2}$ R. Devlin ${ }^{1,3}$.

T. Diaz ${ }^{2}$ T. Dickerson ${ }^{1,19}$ - C. Dickson ${ }^{1,13}$ - A. Diekmann ${ }^{8,20}$ - F. Diggs ${ }^{1,13}$ - C. Duncan' ${ }^{1}$

A. Figueroa-Vinas ${ }^{1}$ - C. Firman ${ }^{1,21} \cdot$ M. Freeman ${ }^{2}$. N. Galassi ${ }^{1,3} \cdot$ K. Garcia ${ }^{1}$.

G. Goodhart ${ }^{2}$ - D. Guererro ${ }^{2}$. J. Hageman ${ }^{1}$ - J. Hanley ${ }^{2}$ - E. Hemminger ${ }^{1,22}$.

M. Holland ${ }^{1}$ M. Hutchins ${ }^{2}$ - T. James ${ }^{1}$ - W. Jones ${ }^{1,23}$ - S. Kreisler ${ }^{1,24} \cdot$ J. Kujawski ${ }^{1,25}$.

V. Lavu ${ }^{1,10}$ - J. Lobell ${ }^{1}$ E. LeCompte ${ }^{12}$ - A. Lukemire ${ }^{26}$ - E. MacDonald ${ }^{1}$.

A. Mariano $^{1}$ - T. Mukai ${ }^{4}$ K. Narayanan ${ }^{1}$ - Q. Nguyan ${ }^{1}$ - M. Onizuka ${ }^{1,27}$.

W. Paterson ${ }^{1,28} \cdot$ S. Persyn ${ }^{2}$ B. Piepgrass ${ }^{2}$. F. Cheney ${ }^{1}$ - A. Rager ${ }^{1,17}$.

T. Raghuram ${ }^{1,19} \cdot$ A. Ramil ${ }^{1}$. L. Reichenthal ${ }^{1} \cdot$ H. Rodriguez $^{2} \cdot$ J. Rouzaud $^{12}$.

A. Rucker ${ }^{1,13}$ - Y. Saito ${ }^{29}$ - M. Samara' J.-A. Sauvaud $^{12}$ - D. Schuster ${ }^{1,30}$.

M. Shappirio ${ }^{1}$ K. Shelton ${ }^{1,19}$ - D. Sher ${ }^{1,31}$ - D. Smith ${ }^{1,10}$ - K. Smith ${ }^{2}$ - S. Smith ${ }^{1,17}$.

D. Steinfeld ${ }^{1} \cdot$ R. Szymkiewicz ${ }^{1} \cdot$ K. Tanimoto ${ }^{5} \cdot$ J. Taylor $^{2} \cdot$ C. Tucker ${ }^{1,32} \cdot$ K. Tull $^{1,33}$. A. Uhl ${ }^{1,3} \cdot$ J. Vloet $^{2} \cdot$ P. Walpole ${ }^{1,2} \cdot$ S. Weidner ${ }^{2} \cdot$ D. White ${ }^{2} \cdot$ G. Winkert ${ }^{1} \cdot$ P.-S. Yeh ${ }^{1}$. M. Zeuch ${ }^{1,13}$

\footnotetext{
C. Pollock

craig@denaliscientific.org

1 NASA Goddard Space Flight Center, Greenbelt, MD, USA

2 Southwest Research Institute, San Antonio, TX, USA

3 SGT Inc., Greenbelt, MD, USA

4 Institute for Space and Astronautical Sciences, Sagamihara, Japan

5 Meisei Electric Company, Ltd., Isesaki, Japan

6 University of Maryland, College Park, MD, USA

7 Millenium Engineering, Arlington, VA, USA

8 NASA Marshall Space Flight Center, Huntsville, AL, USA

9 Oak Ridge Associated Universities, Oak Ridge, TN, USA

10 Orbital Sciences Corp., Beltsville, MD, USA

11 Stellar Solutions Inc., Chantilly, VA, USA

12 Institute for Research in Astrophysics and Planetology, Toulouse, France

13 Northrup Grumman, Hyattsville, MD, USA
} 


\begin{abstract}
The Fast Plasma Investigation (FPI) was developed for flight on the Magnetospheric Multiscale (MMS) mission to measure the differential directional flux of magnetospheric electrons and ions with unprecedented time resolution to resolve kinetic-scale plasma dynamics. This increased resolution has been accomplished by placing four dual 180-degree top hat spectrometers for electrons and four dual 180-degree top hat spectrometers for ions around the periphery of each of four MMS spacecraft. Using electrostatic fieldof-view deflection, the eight spectrometers for each species together provide 4pi-sr field-ofview with, at worst, 11.25-degree sample spacing. Energy/charge sampling is provided by swept electrostatic energy/charge selection over the range from $10 \mathrm{eV} / \mathrm{q}$ to $30000 \mathrm{eV} / \mathrm{q}$. The eight dual spectrometers on each spacecraft are controlled and interrogated by a single block redundant Instrument Data Processing Unit, which in turn interfaces to the observatory's Instrument Suite Central Instrument Data Processor. This paper describes the design of FPI, its ground and in-flight calibration, its operational concept, and its data products.
\end{abstract}

Keywords Magnetospheric Multiscale · Magnetic reconnection · Particle instrumentation · Electron spectrometer · Ion spectrometer · Instrument characterization and calibration

\title{
1 Introduction
}

\subsection{Reconnection Science}

NASA's Magnetospheric Multiscale (MMS) mission is focused on the phenomenon of magnetic reconnection occurring in many astrophysical settings, including Earth's magneto-

\footnotetext{
14 Florez Engineering, LLC, Laurel, MD, USA

15 MEI Technologies, Houston, TX, USA

16 Global Programming Solutions, Fairfax, VA, USA

17 Catholic University of America, Washington, DC, USA

18 Johns Hopkins Applied Physics Laboratory, Laurel, MD, USA

19 ASRC AS and D, Inc., Beltsville, MD, USA

20 Jacobs ESSSA Group, Huntsville, AL, USA

21 Prototype Productions, Inc., Ashburn, VA, USA

22 Ares Technical Services Corp., Greenbelt, MD, USA

23 Bandwidth Solutions, Inc., McLean, VA, USA

24 The Hammers Company, Greenbelt, MD, USA

25 Siena College, Loudonville, NY, USA

26 Space Power Electronics, Inc., Kathleen, GA, USA

27 Teledyne Brown Engineering, Huntsville, AL, USA

28 NASA Headquarders, Washington, DC, USA

29 YS Design, Tokyo, Japan

30 Vantage Systems, Inc., Lanham, MD, USA

31 Design America, Inc., College Park, MD, USA

32 Global Science and Technology, Inc., Greenbelt, MD, USA

33 Jackson and Tull, Greenbelt, MD, USA
} 
sphere in both the upstream magnetopause and at sites downstream of Earth in the geomagnetic tail. Magnetic reconnection is responsible for many dynamic processes on the sun and other stars, at the interfaces between stellar environments and the local interstellar medium, and at the interfaces between galaxies and the intergalactic medium. Indeed, wherever disparate magnetic fields are brought together in the presence of plasma, magnetic reconnection may be active. Magnetic reconnection is also active in magnetized laboratory plasmas. In these environments, the process impedes the coherent plasma containment processes necessary to the achievement of controlled nuclear fusion. Thus, understanding the processes responsible for the occurrence of magnetic reconnection is of fundamental importance, both to the understanding of our natural universe and to the applied objective of developing energy generating capacity based on controlled nuclear fusion.

Reconnection is common in many disparate locations, both in space and in terrestrial laboratories, but it is within Earth's magnetosphere where it is most feasible to perform the spatially resolved in situ measurements necessary to understand the process fully. In situ measurements are not feasible on the solar surface or within astrophysical plasmas. Nor can definitive, spatially resolved in situ measurements of the properties and dynamics of reconnecting laboratory plasmas be performed at the present time (scale sizes are too small).

For these reasons, the MMS mission was conceived to visit reconnection sites in Earth's magnetosphere with a contingent of four identically instrumented spacecraft flying in a close tetrahedral formation. The tetrahedral geometry allows simultaneous determination of plasma and field properties at four spatial locations, in turn enabling specification of spatial gradients in the plasma properties.

\subsection{Breaking the Spin Rate Limit}

The Fast Plasma Investigation (FPI), developed for flight on MMS, is dedicated to the rapid measurement of the phase space densities of electrons and positive ions within and near sites in Earth's magnetosphere where reconnection occurs. The mission requirement is to measure the $3 \mathrm{D}$ ion and electron phase space distributions at $150 \mathrm{~ms}$ and $30 \mathrm{~ms}$, respectively. This high temporal resolution in 3D phase space measurements is unprecedented in the history of scientific space flight and by far the single biggest driver on the design and implementation of the experiment.

Our approach has been to distribute many high-speed sensors (eight for each species, packaged in pairs as 'dual spectrometers') around the spacecraft perimeter so that full azimuthal sampling need not depend on the spin of the spacecraft as has been common for magnetospheric missions. This deployment strategy allows measurement of the 3D ion and electron phase space densities at speeds limited primarily by the stepper speeds of our high voltage power supplies and the counting statistics possible within the very short accumulation intervals imposed.

An unavoidable consequence of this approach is that the 3D phase space density for either species at the spacecraft location must be stitched together from the measurements of eight different spectrometers. Each group of four dual spectrometers includes twelve independent high voltage power supplies: eight for detector bias and four (each with three outputs) for energy and angle selection, presenting an inter-calibration challenge that was managed as part of the instrument development process and will continue to be managed in flight.

Provision of the FPI has been a highly complex endeavor, with a large volume of flight hardware developed, assembled and tested. Despite the size of the undertaking, we were able to successfully execute with high quality owing to the innovativeness, resilience and dedication of the FPI team at all levels. 


\subsection{Science Objectives}

The overarching objective of the MMS mission is to understand the microphysics of magnetic reconnection by determining the kinetic processes occurring in the electron diffusion region that are responsible for collisionless magnetic reconnection, especially how reconnection is initiated.

MMS will address three specific objectives:

- Determine the role played by electron inertial effects and turbulent dissipation in driving magnetic reconnection in the electron diffusion region.

- Determine the rate of magnetic reconnection and the parameters that control it.

- Determine the role played by ion inertial effects in the physics of magnetic reconnection.

The associated mission-level science and measurement requirements specific to FPI are:

(1) Resolve plasma conditions in the reconnection diffusion region on electron and ion time scales, including plasma flows, currents, temperatures and heat flow. The necessary time scales are those on which features with electron length scales $(\sim$ few $\mathrm{km})$ and ion length scales $(\sim 200 \mathrm{~km})$ pass over the observatories, not necessarily those associated with local electron and ion gyro periods or plasma periods. For a typical magnetopause speed of $50 \mathrm{~km} / \mathrm{s}$, these time scales are of the order of $100 \mathrm{~ms}$ and $4 \mathrm{~s}$ for electrons and ions, respectively.

(2) Measure the decoupling of ions from the magnetic field across the reconnection region. This means tracking both the local ion convection velocity (performed by FPI) and the local ExB plasma convection velocity (performed by the MMS Fields Investigation) to document their departure from equality with one another.

(3) Measure the contribution of ions to plasma turbulence generation and determine the resulting ion heating rates.

(4) Resolve thin electron layers and associated currents. The thin current sheets centered in the electron diffusion region that are responsible for large-scale magnetic reversal have not been adequately resolved by previous missions. Several such layers are likely to exist in various forms relevant to reconnection. For example, very thin high speed current carrying electron flows near the boundary of the reconnection exhaust region are predicted by simulations (Daughton et al. 2006) and myriad instances of thin electron current sheets may be found in association with the reconnection region and in fields of turbulence, perhaps themselves rich with turbulent reconnection sites.

(5) At the smallest scales, measure the decoupling of electrons from the magnetic field within the electron diffusion regions. Tracking the electron convection velocity and its comparison with the Fields suite's measurement of ExB is the crucial observation.

(6) Resolve the causes of phase-space density modifications and energization of energetic electrons and ions.

(7) Determine ion inflow and outflow velocities and plasma pressure and anisotropy gradients. Accurate determination of boundary orientations and speeds as well as of ion and electron flows are required to perform these measurements.

(8) Determine mass flow rates across the magnetopause during reconnection. Ion flows into the magnetosphere can often be assumed to have solar wind-like composition of $96 \%$ protons and $>3.9 \%$ alpha particles. In these cases, accurate determination of mass flux across the magnetopause into the magnetosphere requires only determination of corresponding ion number flux. For escaping ion flows or other cases where the measurement of the composition is essential, the Hot Plasma Composition Analyzer (HPCA) will contribute this information at a $10 \mathrm{~s}$ cadence (Young et al. 2014). 
(9) Determine divergence of energy flux across the reconnection boundary. FPI will provide measurements of the charged particle differential directional energy flux within the energy range of $10 \mathrm{eV}-30 \mathrm{keV}$. Integrating this over energy and angle yields the energy flux vector at a point. The MMS constellation as a whole then provides the means to derive the local divergence.

(10) Map magnetic topology and resolve structures of the electron and ion diffusion regions.

(11) Resolve reconnection acceleration sites through triangulation using remote measurements of accelerated electrons and ions.

(12) Determine the motion and orientation of boundaries such as the magnetopause and the magnetotail current sheets.

These scientific measurement requirements drove the specific instrument requirement for the investigation, including energy range (10 eV-30 keV), energy resolution (20\% or better), angular resolution $\left(15^{\circ}\right.$ or better), sensitivity $\left(G>5 \times 10^{-4} \mathrm{~cm}^{2} \mathrm{sreV} / \mathrm{eV}\right.$ for ions and $G>$ $1 \times 10^{-4} \mathrm{~cm}^{2} \mathrm{sreV} / \mathrm{eV}$ for electrons), and time resolution $(150 \mathrm{~ms}$ for ions and $30 \mathrm{~ms}$ for electrons).

In Sect. 2, we describe the approach to the fast plasma measurements required for MMS. In Sect. 3, we describe the detailed implementation. Elements shared in common for the ion and electron measurements are described in common, while subsections are provided for both the electron measurements and the ion measurements, as well as for the associated command and data processing hardware. Section 4 describes central elements of the instrument calibration, both in the laboratory and in flight, and provides examples and summary results from the ground calibration. In Sect. 5, we describe the FPI operations, which include both routine operations and regularly scheduled in-flight calibration operations. The FPI ground system and various data handling systems and protocols are presented in Sect. 6. Section 7 provides a summary and set of conclusions.

\section{FPI Measurement Approach}

\subsection{Notes About Geometry Conventions}

Following are the conventions used to refer to angles in the context of the spacecraft geometry:

(1) Polar angle $\theta$ such that $0^{\circ} \leq \theta \leq 180^{\circ}$, opens from the spacecraft $+\mathrm{Z}$ axis (nominally the spin axis)

(2) Azimuth angle, $\phi$, such that $0^{\circ}<\phi<360^{\circ}$, corresponds to an angle in the spacecraft $\mathrm{X}-\mathrm{Y}$ plane, opening from the spacecraft $+\mathrm{X}$ axis with a positive right hand rotation about the spacecraft $+\mathrm{Z}$ axis.

Regarding the FPI sensor, we adopt the conventions:

(1) The FPI sensor convention and the spacecraft convention are identical to within a phase offset $\Delta \phi$, which can be context dependent.

(2) The FPI sensor polar angle is the sensor's 'pixel-to-pixel' imaging dimension, and a FPI sensor azimuth angle is the sensor's deflected angle dimension. The sensors are mounted on the spacecraft so that instrument polar and azimuth angles correspond to spacecraft polar and azimuth angles. 
Fig. 1 Illustration of basic top hat plasma spectrometer geometry-top view and cross section view. First introduced by Carlson et al. (1982), who referred to it as a symmetric quadrisphere, this top hat ESA design has been used extensively. This illustration adapted from his paper
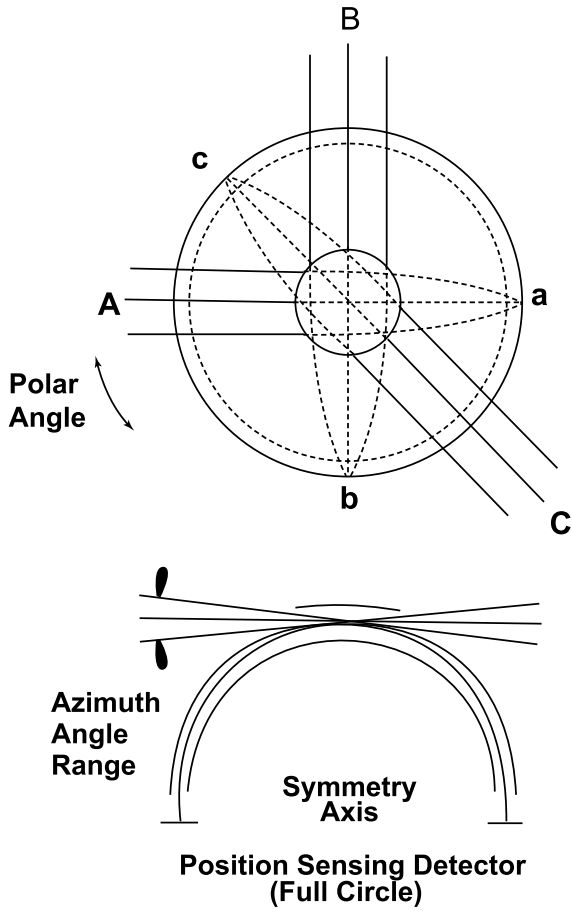

\subsection{The Top Hat Plasma Spectrometer}

FPI utilizes top hat electrostatic analyzers (ESAs) to filter for phase space locations and Micro Channel Plates (MCPs) followed by pulse height discriminators and counters to measure the differential directional flux of electrons and ions at those phase space locations. The specific configuration employed was first widely introduced to the heliophysics community by Carlson et al. (1982), who referred to it as a symmetric quadrisphere, as illustrated in Fig. 1. The top hat ESA geometry has been used extensively since its introduction.

In its simplest configuration the top hat ESA consists, quoting Carlson et al. (1982), "of three concentric spherical section elements: an inner hemisphere with radius $R_{1}$ that is driven with the voltage $V_{E S A}$, an outer hemisphere with radius $R_{1}+\Delta_{1}$ which contains a circular hole subtending a half-angle $\Theta$, (in upper case here in contrast with Carlson et al.'s (1982) notation, in order to differentiate it from FPI's observation polar angle, $\theta$ ) and a small "top cap" section of radius $R_{1}+\Delta_{1}+\Delta_{2}$, which, in conjunction with the outer hemisphere, defines the cylindrical entrance aperture".

Application of $V_{E S A}$ produces an electric field between the nested hemispheres that guides an appropriately energetic and directional charged particle between the plates to the annular exit aperture, below which a position-sensitive detector resides. A stepped $V_{E S A}$ sequence is used to acquire count rates within a band pass centered at a sequence of energy/charge $(\epsilon)$ that is proportional to the applied voltage. The constant of proportionality, $k \epsilon$, between the selected energy/charge and $V_{E S A}$ is referred to as the analyzer constant and is determined by the ratio $\Delta_{1} / R_{1}$. The relation between the applied ESA voltage and the selected particle energy is given as

$$
\epsilon=\epsilon_{0}+k \epsilon * V_{E S A}
$$


where $\epsilon$ is the selected energy per charge, $k \epsilon$ is the analyzer constant and $\epsilon_{0}$ is a small constant offset arising from the electronics.

The hemispherical top hat ESA described is the original and simplest configuration (Carlson et al. 1982). A small but important variation involves introduction of a nearly hemispherical toroidal geometry, first introduced by Young et al. (1988). In this case, the selected energy per charge remains a linear function of applied ESA voltage. The toroidal geometry places the electrostatic optical focus slightly further downstream along the charged particle path in the vicinity of the ESA exit aperture and provides a larger sensitivity per unit volume (Young et al. 1988). In the case of FPI, the Dual Electron Spectrometer (DES) utilizes the hemispheric geometry and the Dual Ion Spectrometer (DIS) incorporates the toroidal geometry.

Note that a top hat ESA allows simultaneous observation over an angular range of up to $360^{\circ}$ in the plane perpendicular to the symmetry axis. Particles arriving from different angles around the top hat field of view (FOV) are directed to corresponding locations around the annular exit aperture and can be differentiated using an annular position-sensitive detector just downstream of the ESA exit aperture. FPI only uses $180^{\circ}$ of the top hat's potential $360^{\circ}$ FOV, and we refer to this angular dimension as the polar angle because it corresponds to the angle opening from the spacecraft Z-axis consistent with the usage of Carlson et al. (1982) and Fig. 1.

Further, the nominal FOV of a top hat ESA lies within a band pass centered near the plane perpendicular to the instrument symmetry axis. FPI employs additional curved electrodes located above and below that plane and upstream of the top hat entrance aperture to achieve FOVs away from that nominal view plane. This deflected angle is referred to here as the azimuth angle, consistent with the notion of the spacecraft azimuthal coordinate corresponding to spin phase. Previous examples of space plasma instruments which have used such FOV deflection can be found in Carlson et al. (2001), Young et al. (2007), Burch et al. (2007) and McComas et al. (2013).

The angular deflection achieved in this manner is typically a linear function of the ratio of the voltage difference between the two deflecting electrodes $\left(V_{D E F}\right)$ and the currently selected energy per charge as determined by the current value of $V_{E S A}$. That is,

$$
\phi=\phi_{0}+k_{\phi} * V_{D E F} / \varepsilon
$$

where $\phi$ is the deflected angle, $k_{\phi}$ is the deflection constant and $\phi_{0}$ is a small offset arising from the electronics. The linear relation expressed in Eq. (2) applies over a limited range of azimuth, which is easily accommodated in the FPI application.

Ideally, the pixel count rate at any $V_{E S A}$ is proportional to $\left\langle\boldsymbol{j}^{\prime}\right\rangle$, the average differential directional energy flux (units: $\mathrm{eV} /\left(\mathrm{cm}^{2} \mathrm{sr} \mathrm{seV}\right)$ ) carried by particles at the aperture and within the pixel FOV and energy pass bands at the current $V_{D E F}$ and $V_{E S A}$. The constant of proportionality here is referred to as the pixel geometric factor $G$, which may be approximated and conceptualized as:

$$
G \approx A_{\text {eff }} \times \delta \Omega \times \delta \varepsilon / \varepsilon
$$

where $A_{\text {eff }}$ is the effective aperture area for the pixel, $\delta \Omega$ is the $2 \mathrm{D}$ solid angle band pass and $\delta \varepsilon / \varepsilon$ is the band pass in energy/charge. The effective aperture area includes factors such as the non-unity transmission of screens in the flight path and the non-unity efficiency of the detection system. Rigorous descriptions of the FPI pixel geometric factor, appropriate techniques for its estimation using numeric particle ray tracing, its experimental determination through laboratory calibration, and its use in interpreting space plasma measurements have been provided by Collinson et al. (2012). For FPI, the Analyzer Constants $\left(k \epsilon\right.$ and $\left.k_{\phi}\right)$, 
Fig. 2 Each FPI $180^{\circ}$ polar angle top hat is mounted so that the $180^{\circ}$ fan spans from spacecraft spin axis to anti-spin axis. Each sensor is oriented so that the 16 pixels (each nominally $11.25^{\circ}$ wide) of its $180^{\circ} \mathrm{FOV}$ are viewing radially in velocity space, spanning the $180^{\circ}$ pole-to-pole range in the spinning spacecraft reference frame

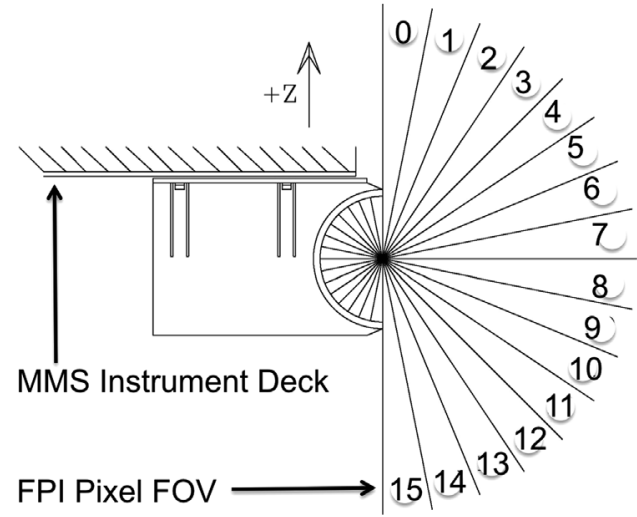

Geometric Factors, angular FOV band passes, and energy band passes were predicted with numeric ray tracing and subsequently measured for every flight instrument using laboratory calibrations.

\subsection{Top Hat Approach for MMS}

\subsubsection{Half Polar Angle FOV Configuration}

The required time resolution, $30 \mathrm{~ms}(150 \mathrm{~ms})$ for electrons (ions), is by far the most challenging requirement for FPI. This is 333 (67) times faster than could be achieved simply by waiting for the spinning spacecraft to rotate through a half spin. Spacecraft spin has typically set the effective time resolution achievable for 3D plasma measurements in the past. Notable exceptions include The Electron and Ion Plasma Spectrometer on FAST (Carlson et al. 2001), the HYDRA instrument on Polar (Scudder et al. 1995) and more recently, the JADE electron spectrometer on Juno (McComas et al. 2013).

A $180^{\circ}$ polar angle top hat mounted so that the $180^{\circ}$ fan spans from spacecraft spin axis to anti-spin axis, as illustrated in Fig. 2, is the basis for the FPI sensors. The FPI approach to meeting its high time resolution requirements is to deploy eight $180^{\circ}$ top hat sensors for electrons and eight $180^{\circ}$ top hat sensors for ions around each spacecraft perimeter. Each sensor is oriented so that the 16 pixels (each nominally $11.25^{\circ}$ wide) of its $180^{\circ} \mathrm{FOV}$ are viewing radially in velocity space, spanning the $180^{\circ}$ pole-to-pole range in the spinning spacecraft reference frame. The pole-to-pole pixel array and the distribution of eight spectrometers around the spacecraft azimuth provide simultaneous sampling in these (polar and azimuthal) orthogonal angular dimensions.

\subsubsection{Dual Spectrometers at $90^{\circ}$ Spacing}

Each of the four spacecraft carries eight FPI electron spectrometers and eight FPI ion spectrometers. These are packaged in pairs in back-to-back configuration, as dual spectrometers for each species: DES and DIS. Four dual spectrometers for each species are placed around the MMS instrument deck perimeter at $90^{\circ}$ intervals. For each dual spectrometer, the two (undeflected) $180^{\circ} \mathrm{FOV}$ fans are separated by $45^{\circ}$ in azimuth. 
Fig. 3 To meet temporal requirements, thirty-two azimuthal fields of view using eight spectrometers (four dual spectrometers) are deployed around each MMS observatory perimeter. Nominally identical fields of view are provided for electrons and for ions. Eight spectrometers for each species, each exercising four deflected fields of view, yields thirty-two azimuth samples for each species

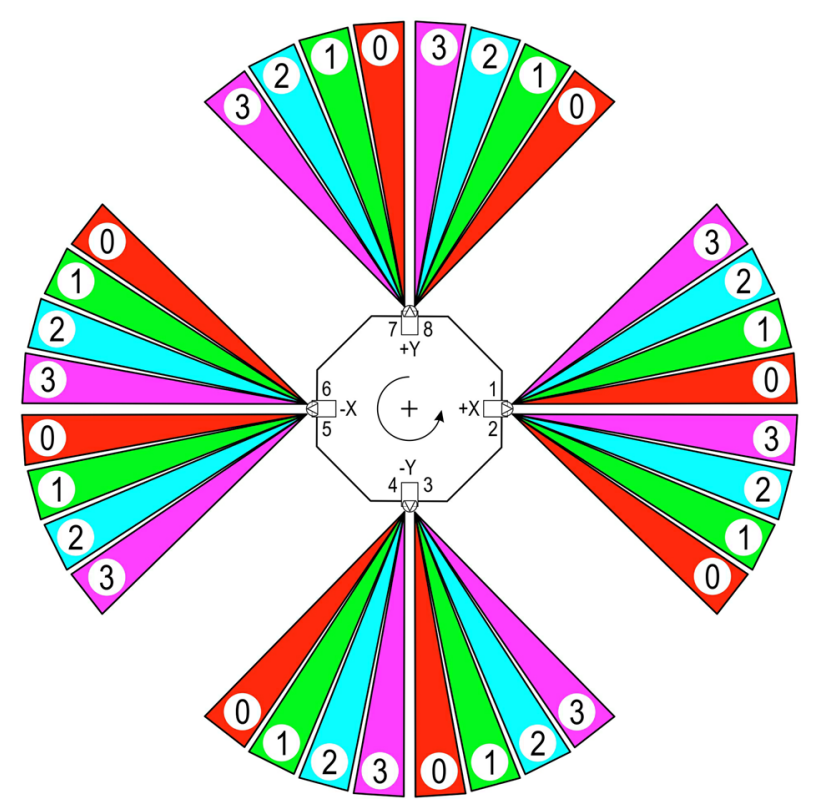

\subsubsection{Deflection for Full View of Sky}

Together, the eight spectrometers for each species provide eight undeflected FOVs around the spacecraft azimuth, or spin plane. This provides $45^{\circ}$ sampling of the plasma velocity phase space in spacecraft azimuth, which on its own is insufficient. Therefore, the FPI spectrometers also incorporate electrostatic FOV deflection, such that center of the azimuth angle FOV of each spectrometer may be deflected in spacecraft azimuth by up to $\pm 16.875^{\circ}$. This is accomplished by applying positive voltage to curved electrodes located just inside of the sensor entrance apertures (the positive polarity prevents production and emission of energetic photoelectrons). The deflection electrodes steer incoming particles from selected azimuth directions toward the top hat aperture. In FPI's Fast Survey mode, each of the eight ion and electron spectrometers samples four azimuths, providing a total 32 azimuthal samples separated by $11.25^{\circ}$ for each species. This azimuth coverage is illustrated in Fig. 3 , where the azimuth FOV of a set of either DIS or DES are illustrated in the spacecraft reference frame. The designations $0,1,2,3$ refer to deflector zone definitions that will be more fully described below. The colors in Fig. 3 denote common deflection states in the sensor reference frame (i.e., down toward MCP plane or up away from MCP plane).

A characteristic of the pole-to-pole FOV is that only the equatorial pixels view an extent in spacecraft azimuth equal to their intrinsic pixel resolution. Off-equatorial pixels view a larger range of azimuth due to the cosine effect in the spherical polar coordinate system. The most poleward viewing pixels view a large swath of azimuth, approaching $\pi$ radians for the rectangular pixels with boundaries at polar angles of $0^{\circ}$ and $180^{\circ}$. These effects are accounted for in the results derived from the measurements.

\subsection{FPI 3D Plasma Observation Sequence in Fast Survey Mode}

The FPI azimuth deflection scheme provides for 32 azimuth $\times 16$ polar phase space angular samples. The energy sweep provides the radial component of spherical velocity space sampling. Importantly, the 16 spacecraft polar angles are all sampled simultaneously at every 
step using the discrete anode arrays, while the 32 spacecraft azimuth angles are sampled in four sequential sets of eight.

All spectrometers target the same energy and deflected angle sets in the spacecraft frame of reference, providing energy coverage from $10 \mathrm{eV}$ to $30 \mathrm{keV}$ and full $2 \mathrm{D}$ angle coverage. The individual spectrometers in each pair are mounted top-to-top and are therefore sampling opposite angle sets in their own reference frames.

This FPI measurement approach means that the 3D velocity distribution functions for electrons and ions will each be assembled using eight different spectrometers, four different ESA HV stepping supplies, and eight different deflection HV stepping supplies. While common sets of energies and angles are targeted, the spectrometers and their HV stepping supplies all have slightly different properties that, in the case of the supplies, will evolve differently through mission life. To compensate, the effects of the voltage commands used in targeting these energy and angle sets will be monitored closely and individual HV offsets will be applied over time for each of the spectrometers. The samples in velocity space have not only absolute but also relative uncertainties within a single 3D distribution. For this reason, separate sweep table constants are maintained for each dual spectrometer tailored to the individual HV supplies; these will be maintained and updated as necessary. The appropriate voltages are initially based on careful laboratory calibrations and will be updated throughout the life of the mission as determined necessary by inflight calibration activities.

\section{FPI Implementation}

The FPI consists of four DES, four DIS and a single Instrument Data Processing Unit (IDPU) on each of the four MMS spacecraft. A simplified block diagram is shown in Fig. 4. The dual spectrometers receive low voltage power and are controlled by, and pass data to, the IDPU. The IDPU, in turn, is controlled by the Central Instrument Data Processor (CIDP) and passes all science and housekeeping data to it for storage and preparation for downlink.

The layout of the instrument deck has one each DES and DIS located next to each other every $90^{\circ}$ around the perimeter with their apertures facing radially outward and imaging the $180^{\circ}$ angular space between the spacecraft $\pm Z$ axes into 16 pixels. Figure 5 shows this layout in the case of all four MMS observatories, with identification of the right-hand spacecraft coordinate system and the positions of each DES, DIS, and IDPU by flight unit number. The DES and DIS are fastened to the underside of the deck and the view in Fig. 5 is from the

Fig. 4 Simplified FPI block diagram for a single MMS observatory, showing the relationship between the DES, DIS, IDPU, and CIDP

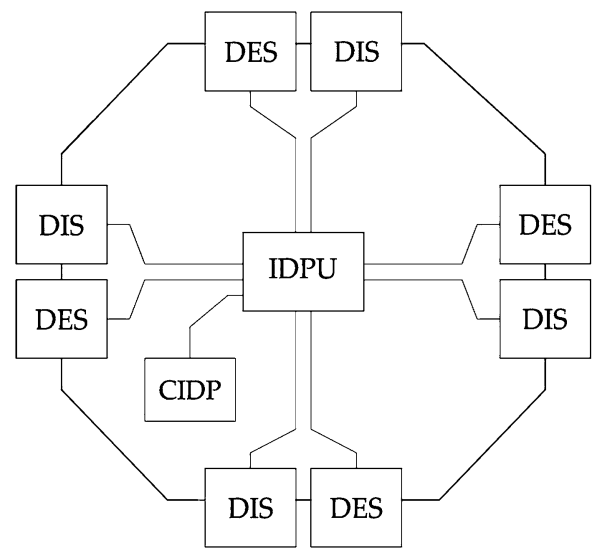



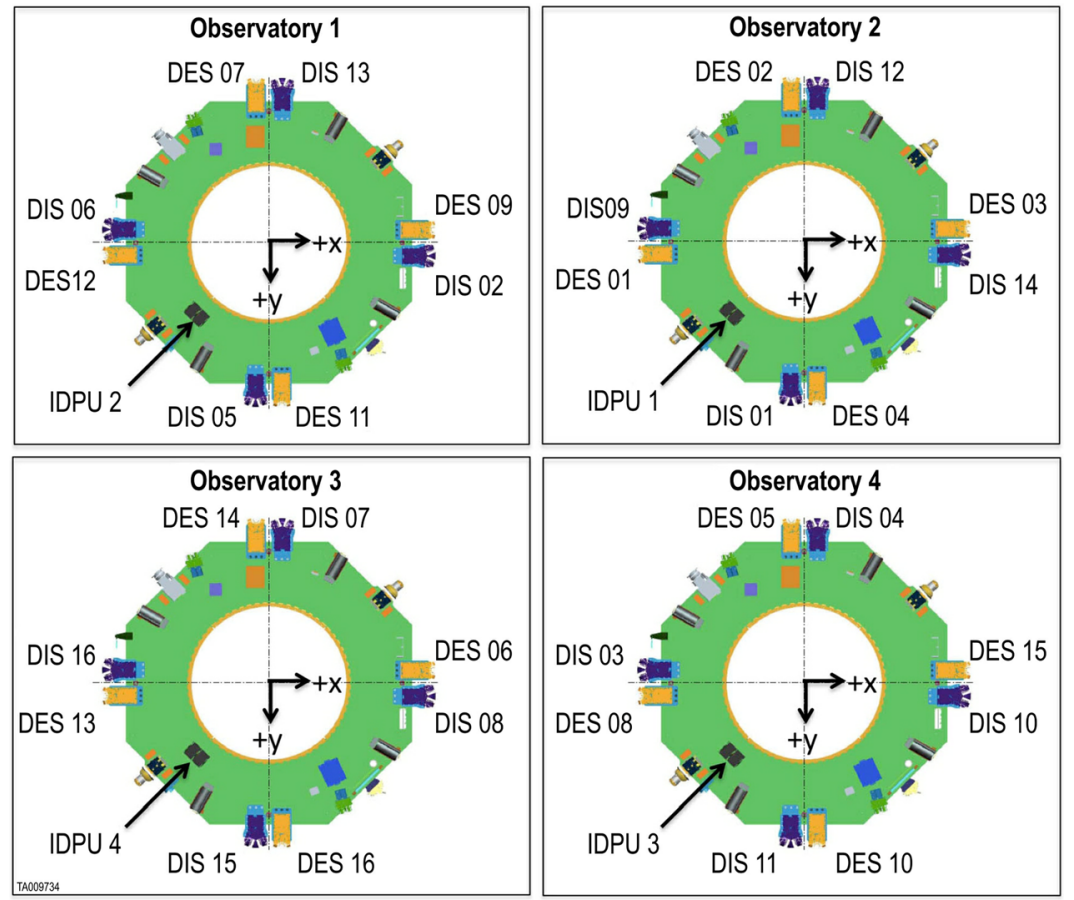

Fig. 5 Layout of FPI elements on each of the four MMS Observatories, with identification of the flight unit numbers in the right-hand spacecraft coordinate system

underside of the deck looking parallel to the spacecraft $+\mathrm{Z}$ axis, which will point nearly northward in flight, perpendicular to the heliospheric ecliptic plane. From the point-of-view of Fig. 5, the spacecraft will spin clockwise.

The single IDPU on each spacecraft is also fastened to the underside of the instrument deck, interior from the perimeter. There is no computer within the FPI either in the dual spectrometers or in the IDPU. All functions are executed by external commands under the control of Field Programmable Gate Arrays (FPGAs) in order to facilitate rapid execution and avoid the considerable resources/complexities required with inflight computers. This limited some flexibilities of functionality and shifted some requirements into flight operations planning and execution. In this implementation, control resides as macros in the payload-level CIDP. Considering all trades regarding the IDPU in hindsight, the FPI team would have preferred a CPU in the design if mass/power resources had permitted.

\subsection{Performance Requirements}

The functional performance requirements to achieve the scientific goals of the MMS mission are listed in Table 1 together with the measured performance across the thirty-two flight units. The spectrometers measure 3D (energy $+2 \mathrm{D}$ angle) electron or ion differential directional flux distributions with very high temporal resolution. The fast time sampling yields high spatial resolution in the presence of fast-moving plasma structures. To robustly cover the energy and angle ranges at burst cadence, electron and ion fluxes are sampled at 16384 different positions in velocity space, representing 512 (16 polar $\times 32$ azimuth) angular lo- 
Table 1 DES and DIS performance requirements and measured performance

\begin{tabular}{|c|c|c|}
\hline Parameter & Requirement & Measured performance \\
\hline \multicolumn{3}{|c|}{ Dual Electron Spectrometers (DES) } \\
\hline Instantaneous polar FOV & $180^{\circ}$ & $180^{\circ}$ \\
\hline Polar angle resolution & $\leq 15^{\circ}$ & $9-15^{\circ}$ \\
\hline Azimuth separation & $45^{\circ} \pm 0.5^{\circ}$ & $45^{\circ} \pm 0.5^{\circ}$ \\
\hline Azimuth FOV deflection & Up to $\pm 17^{\circ}$ & $> \pm 17^{\circ}$ \\
\hline Azimuth FOV width & $\leq 11.25$ & $4-11^{\circ}$ \\
\hline Energy/charge range & $0.01-30 \mathrm{keV}$ & $0.01-30 \mathrm{keV}$ \\
\hline Energy/charge width & $\leq 20 \%$ & $14-20 \%$ \\
\hline Pixel geometric factor & $\geq 1 \times 10^{-4} \mathrm{~cm}^{2} \mathrm{sreV} / \mathrm{eV}$ & $(1-7) \times 10^{-4} \mathrm{~cm}^{2} \mathrm{sreV} / \mathrm{eV}$ \\
\hline 3D time resolution & $30 \mathrm{~ms}$ & $30 \mathrm{~ms}$ (coarser at $7.5 \mathrm{~ms}$ ) \\
\hline Sample integration time & $195 \mu \mathrm{s}$ & $195 \mu \mathrm{s}$ \\
\hline Avg. HV settling time ${ }^{1}$ & $\leq 39 \mu \mathrm{s}(\mathrm{EOL})$ & 24-27 $\mu \mathrm{s}(\mathrm{BOL})$ \\
\hline Total HV settling time ${ }^{1}$ & $\leq 5 \mathrm{~ms}(\mathrm{EOL})$ & $3.1-3.4 \mathrm{~ms}(\mathrm{BOL})$ \\
\hline \multicolumn{3}{|c|}{ Dual Ion Spectrometers (DIS) } \\
\hline Instantaneous polar FOV & $180^{\circ}$ & $180^{\circ}$ \\
\hline Polar angle resolution & $\leq 15^{\circ}$ & $\leq 15^{\circ}$ \\
\hline Azimuth separation & $45^{\circ} \pm 0.5^{\circ}$ & $45^{\circ} \pm 0.5^{\circ}$ \\
\hline Azimuth FOV deflection & Up to $\pm 17^{\circ}$ & $> \pm 17^{\circ}$ \\
\hline Azimuth FOV width & $\leq 11.25$ & $4-6.5^{\circ}$ \\
\hline Energy/charge range & $0.01-30 \mathrm{keV}$ & $0.01-30 \mathrm{keV}$ \\
\hline Energy/charge width & $\leq 20 \%$ & $11-15 \%$ \\
\hline Pixel geometric factor & $\geq 5 \times 10^{-4} \mathrm{~cm}^{2} \mathrm{sreV} / \mathrm{eV}$ & $1-2 \times 10^{-4} \mathrm{~cm}^{2} \mathrm{sreV} / \mathrm{eV}$ \\
\hline $3 \mathrm{D}$ time resolution & $150 \mathrm{~ms}$ & $150 \mathrm{~ms}$ (coarser at $37.5 \mathrm{~ms}$ ) \\
\hline Sample integration time & $1 \mathrm{~ms}$ & $1 \mathrm{~ms}$ \\
\hline
\end{tabular}

${ }^{1} \mathrm{BOL}=$ Beginning of Life; $\mathrm{EOL}=$ End of Life.

cations on each of 32 radial $E / q$ shells. Another 32 radial $E / q$ shells are likewise sampled on alternating parity sweeps, providing 64-step energy coverage at half the burst cadence.

\subsection{The FPI Team and Implementation Approach}

The FPI was collaboratively developed by institutions in the U.S., Japan and France, with Goddard Space Flight Center (GSFC) as the Lead Co-Investigator institution providing overall leadership for development. GSFC provided development and delivery of the Dual Electron Spectrometers (DES) and the Instrument Data Processing Units (IDPU), as well as operation and calibration procedures and associated flight software in the form of CIDP macros. The Dual Ion Spectrometers (DIS) were developed by the Japanese Meisei Electric Co., Ltd. under contract to Southwest Research Institute and with the guidance and direction both from co-Investigators at the Japanese Institute for Aeronautics and Astronautic (ISAS) and from GSFC. The Low Voltage Power Converters (LVPC) in the DES, DIS and IDPU were developed by the U.S. Space Power Electronics, Inc. The Southwest Research Institute developed the DES and DIS High Voltage Power Supplies (HVPS). GSFC procured the DES MCPs from Photonis USA and performed the preconditioning and testing for flight. The 


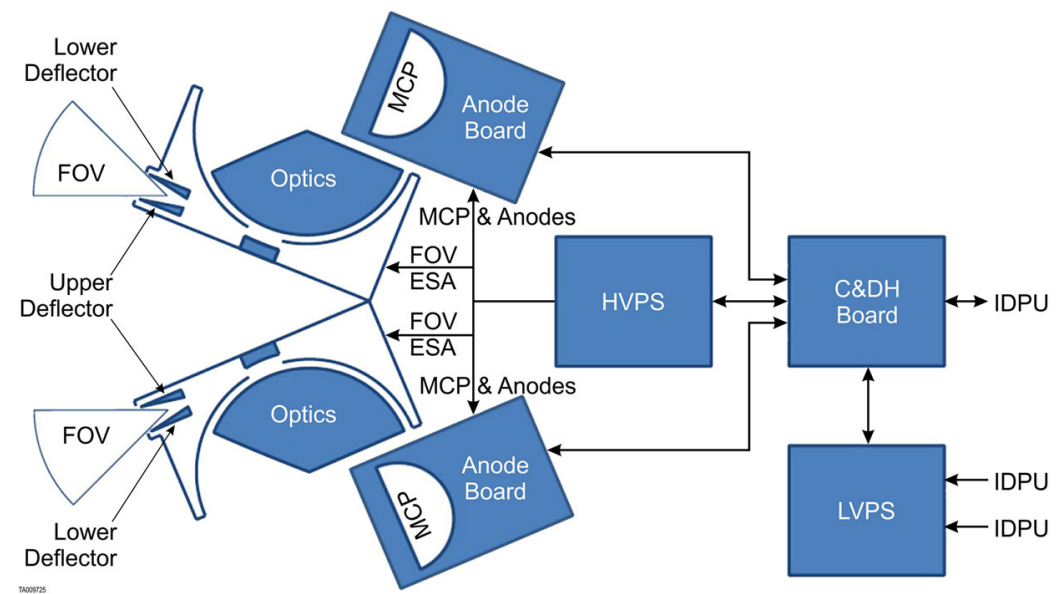

Fig. 6 Block diagram of a DxS shows two sensor heads and electronics box. Each sensor head consists of a set of optics and a detection system comprised of an MCP stack assembly and anode board. The electronics box consists of a HVPS, a C\&DH board and a LVPC

DIS MCPs were procured from Photonis France S.A.S. by the French Institut de Recherche en Astrophysique et Planetologie (IRAP). IRAP also conditioned, tested and mounted the DIS MCPs on anode boards provided by Meisei. These MCP/anodes were then re-tested by IRAP before re-delivery to Meisei for integration into the DIS sensors. The thirty-two DES and four IDPUs were functionally and environmentally tested and calibrated at GSFC. The thirty-two DISs were environmentally tested at Mesei and ISAS (including pre- and postenvironmental beam testing) and were calibrated at NASA's Marshall Space Flight Center (MSFC). Additional testing was performed by the GSFC FPI team after the initial deliveries, including live high voltage testing with particle sources late in the MMS observatory-level thermal vacuum sequence and an extended (1500 hours) thermal vacuum testing of all DIS and DES units designed to purge potentially thermally compromised HV801 opto-couplers. GSFC has lead responsibility for FPI Phase E mission activities in close collaboration with the development partner institutions. The magnitude of the FPI development and deployment required a distributed effort and the full expertise and support of each of these institutions.

\subsection{FPI Dual Spectrometers}

The two spectrometer types-DES and DIS-are similar in design. In this section we describe both, referring to them generically as DxS and then further detailing where their characteristics differ as appropriate. A DxS block diagram is shown in Fig. 6. Each DxS consists of two sensors that share a single electronics box; the three elements are fixed to a common baseplate. Each sensor consists of a set of electrostatic optics and a detection system.

\subsubsection{Optical Design}

Deflectors and ESA The optics in each sensor are based on the standard designs described in Sect. 2 and consist of an $E / q$ analyzer-a truncated hemispherical $180^{\circ}$ top hat in the case of DES and a toroidal $180^{\circ}$ top hat in the case of DIS - and a pair of curved deflection 


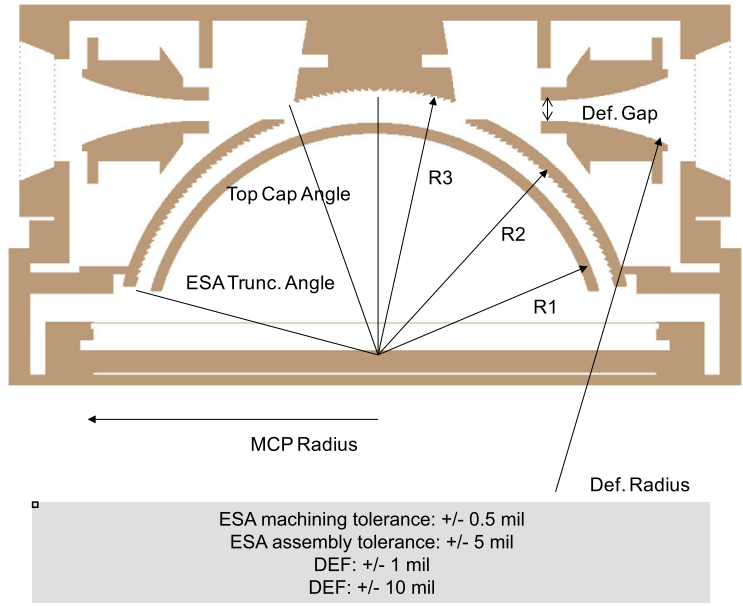

ESA Parameter

Outer radius of inner ESA, R1

Inner radius of outer ESA, R2

Inner radius of top cap, R3

Gap between Inner and Outer ESA

Gap between top cap and Inner ESA

Top cap angle from center to edge

ESA truncation angle

Deflectors inner edge to central axis

Deflector radius

Deflector gap

Deflector angle

MCP Radius

Effective aperture

Value

$34.8 \mathrm{~mm}$

$37.2 \mathrm{~mm}$

$39.6 \mathrm{~mm}$

$2.4 \mathrm{~mm}$

$4.8 \mathrm{~mm}$

$18.0 \mathrm{deg}$

$73.5 \mathrm{deg}$

$25.5 \mathrm{~mm}$

$47.17 \mathrm{~mm}$

$3.14 \mathrm{~mm}$

$22.5 \mathrm{deg}$

$44 \mathrm{~mm}$

$\geq 0.07 \mathrm{~cm}^{2}$

Fig. 7 Diagram of DES optics together with a listing of design parameters

Fig. 8 Diagram of DIS optics

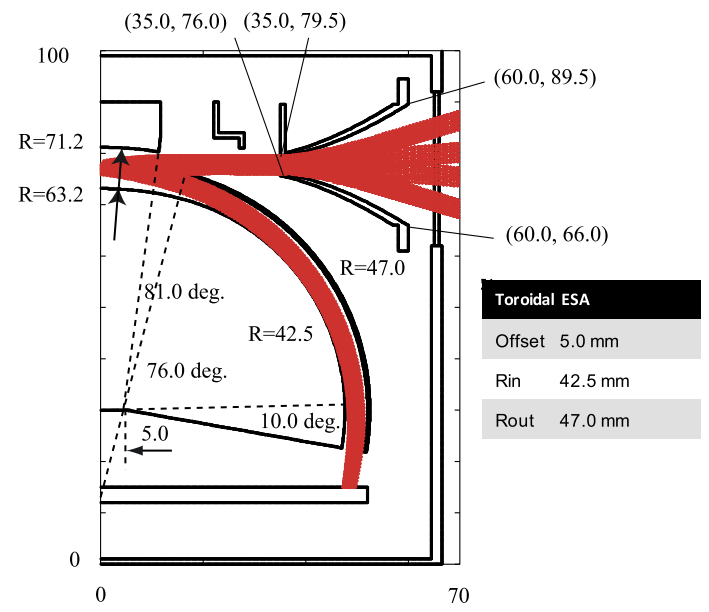

plates upstream of each entrance aperture. To construct a $180^{\circ}$ (spacecraft polar angle) $\times$ $11.25^{\circ}$ (spacecraft azimuth angle) FOV, the azimuthal angle is deflected from $-16.875^{\circ}$ to $+16.875^{\circ}$ in an "umbrella" fashion with respect to the symmetry axis of each top hat. That is, the deflected $180^{\circ} \mathrm{FOV}$ is not strictly in the azimuthal plane but lies on a halfcone, with conical angle equal to the deflection angle. In the case of DES, the deflector electrodes are short $\left(22.5^{\circ}\right)$ circular arcs in cross section, with radius of $47.17 \mathrm{~mm}$ and centers located on $25.5 \mathrm{~mm}$ radius circles centered on the top hat axis and located $12.2 \mathrm{~mm}$ below (lower deflector) and $85.3 \mathrm{~mm}$ above (upper deflector) the center of curvature of the ESA electrodes. The design ESA gap in the case of DIS is $4.5 \mathrm{~mm}$. Precision machined and pin-aligned mechanical design achieved the required uniform spectrometer response at all polar and azimuth view directions across the spectrometer sets. The DES optical design is illustrated in Fig. 7 and the DIS optical design is illustrated in Fig. 8. 


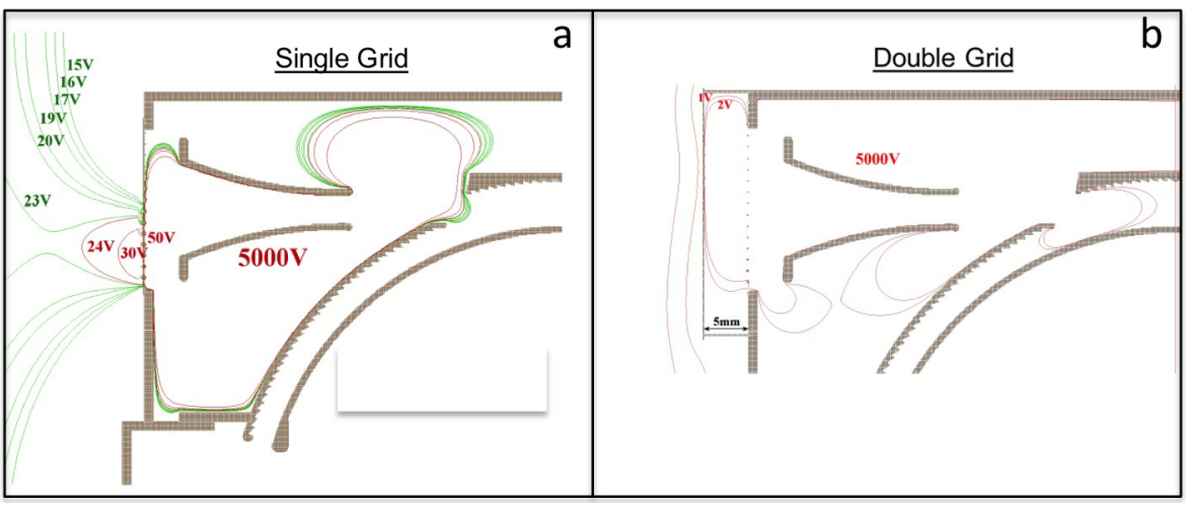

Fig. 9 Diagram showing simulation of electrical field leakage and resulting surface potential with single and double aperture grids

Electrostatic Leakage Control The deflector plates of the DES optics are located very close to the sensor apertures. Without mitigation, the high voltage applied to them would result in leakage of the generated electric field, negatively impacting performance. To confine the electric field within the sensor head, double metal grids were placed across the spectrometer apertures to form a Faraday cage. Figure 9 shows static field simulations performed for a single grid with $90 \%$ transmission and for a double grid with $5 \mathrm{~mm}$ spacing and $90 \%$ transmission (each grid). These simulations demonstrated that the field leakage through the single grid remained higher than the $1 \mathrm{~V}$ maximum surface potential requirement. To achieve the required reduction, a single grid would need to be so dense it would have a particle transmission of only $36 \%$. The figure shows that adding the second grid $5 \mathrm{~mm}$ away from the first meets the requirement with a net particle transmission of $81 \%$. The effect is a small attenuation of the flow of particles through the spectrometer apertures, thereby slightly reducing the sensitivity. Double grids are implemented on both DES and the DIS.

UV Rejection A further design driver was the need to achieve at least 8 (10) orders of magnitude reduction in the detection of UV flux entering the DES (DIS) sensor, which corresponds to the detection of less than one count due to UV photons per sample period. The addition of light trap structures near the entrance apertures was deemed desirable but discarded as an acceptable performance trade in light of the additional complexity and mass required for the 64 spectrometers.

A photon ray-tracing study was performed for the DES optics design by GSFC and iterated as the design evolved. The same analysis was repeated on the DIS design with assistance from ISAS and tests were performed in the ISAS beam chamber to determine the effectiveness of mitigation strategies. The studies were performed using the commercial "FRED Optical Engineering Software" that models photon transmission through the detailed mechanical design, including surface properties. The top five paths by which photons could reach the detectors were determined, all involved reflections from the ESA top cap.

Serrations were designed into the DxS top caps and hemispheres (Fig. 9). The design purposely included serrations over the entire hemisphere to maintain the same average field throughout the energy analyzer even though optical simulations indicated they were strictly necessary only for UV rejection towards the upper part of the hemisphere nearest to the aperture. Figure 10 shows the results of laboratory tests comparing UV sensitivity using 

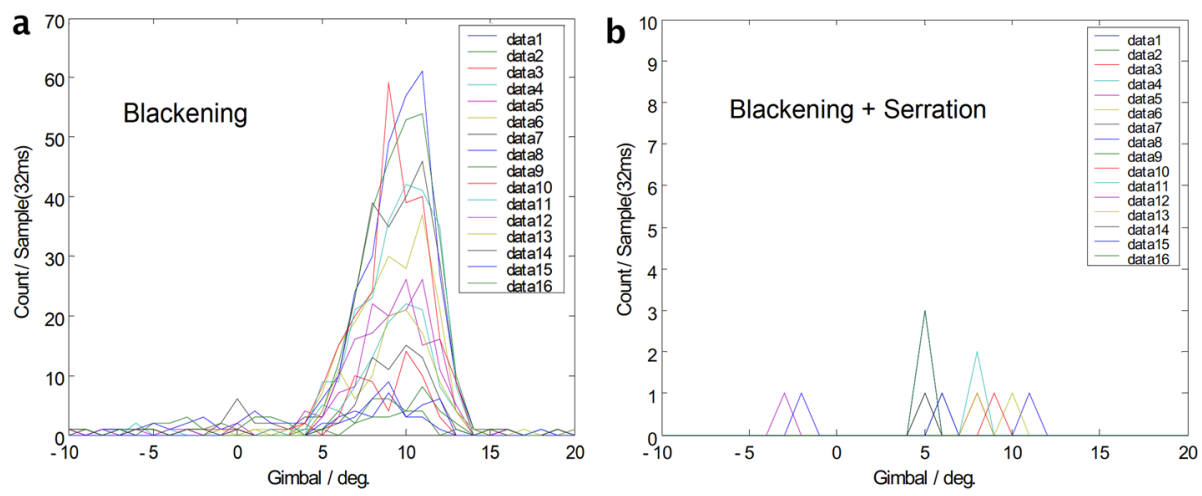

Fig. 10 DIS UV rejection is enhanced by the addition of serrations on the ESA surfaces

blackened DIS ESA surfaces in the cases of serrated and un-serrated ESA electrodes. The angle of incidence of an UV Lyman- $\alpha$ source was varied as detector counts in $32 \mathrm{~ms}$ accumulation intervals were recorded. In Fig. 10a, the test article had smooth un-serrated surfaces, while in Fig. 10b the ESA surfaces (including inner electrode, outer electrode, and topcap electrode) are serrated. Figure 10a shows that as many as 60 counts in $32 \mathrm{~ms}$ were recorded in the case without serrations, which would yield up to two counts per sample in flight. Figure $10 \mathrm{~b}$ demonstrates the UV transmission was reduced by an additional factor of 20 with the addition of serrations on the electrode surfaces. Adding serrations to the inner hemisphere did not substantially contribute to the improved UV rejection performance and since their application would increase the complexity of manufacture the DES inner hemispheres were left smooth. In addition to the serrations, a dark black dendritic finish with very low reflectivity was applied to the optical components. Laboratory measurements demonstrated that this combination of serrations and blackening successfully reduced the UV contamination by more than the required factors.

\subsubsection{Detector Assembly}

Each of the two sensors in a DxS has its own detector system, comprised of entrance shield grids, the MCP stack assembly, and 16 discrete anodes, each serviced by an Amtek A121 hybrid charge sensitive pre-amplifier/discriminator. Detector system components are mounted on the anode board-a multi-layer printed circuit board-on which the anode array itself is formed. Plasma particles passed by the ESA enter the detector assembly through the grid above the MCP stack. The electrical arrangements for DES and DIS are illustrated in Figs. 11 and 12, respectively.

MCP Entrance Shield Grids A shield grid is installed above the first stage MCP input face of all FPI MCP detectors. The intent is to shield the ESA from the electric field of the biased first stage MCP input face, which would otherwise partially penetrate into the lower reaches of the ESA resulting in the transmission or suppression of some particles. This is generally a small effect but can become significant at the lowest energies sampled. The second function of the MCP shield grid is to suppress the backscatter of secondary electrons generated on the input face by the particles transmitted from the ESA. This enhances the detection efficiency of the MCP stack and likely reduces cross talk, as these secondary electrons can migrate to other locations on the MCP or generate $\mathrm{x}$-rays upon impact onto the ESA inner electrode. 


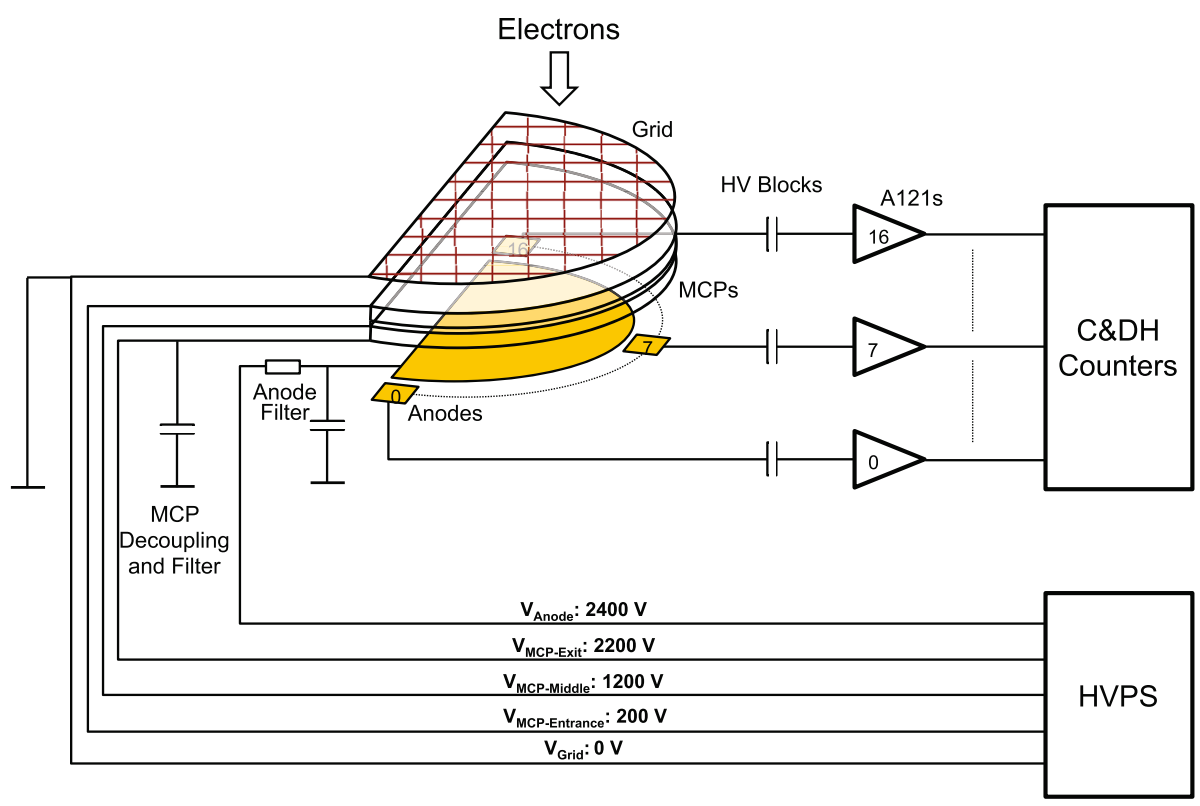

Fig. 11 DES detection system along with its interfaces to the C\&DH and HVPS as housed in the electronics box. Only 3 of the 16 discrete channels are shown

Fig. 12 DIS detection system along with its interfaces to the C\&DH and the HVPS, both of which are housed in the DIS electronic box. Only 4 of the 16 discrete channels are shown

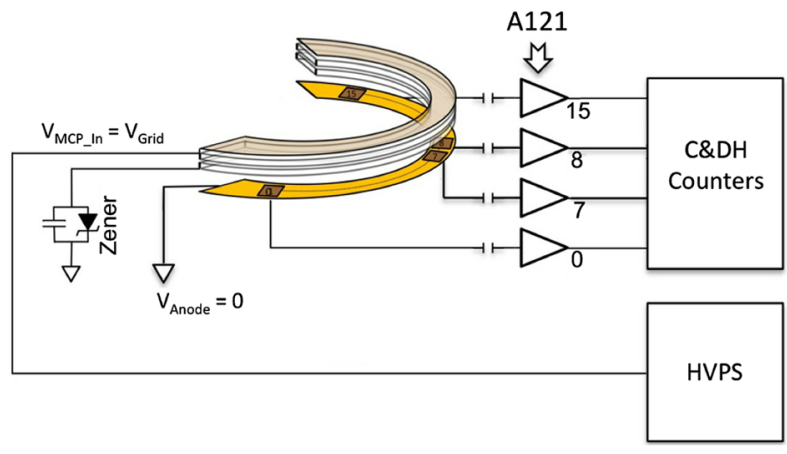

In the case of the DIS, the grid is biased in common with the input surface of the MCP because of concerns about high voltage interactions between the MCP and grid. Ray tracing indicates a somewhat enhanced DIS geometric factor (broadened energy/azimuth angle response) at lower energies due to this field leakage.

MCP Stack Assemblies The MCP stack amplifies each incoming electron or ion into a pulse of outgoing electrons, the magnitude of which (number of electrons per pulse) is variable and characterized with a histogram known as a pulse height distribution. The pulse height distribution depends strongly on the voltage applied across the MCP stack, the MCPs that comprise it, the rate per unit area at which it is emitting pulses and both the immediate (counting rate) and long term history (total counts, contamination) of the MCP stack.

Plasma particles admitted by the ESA and entering the tubes of the top MCP strike the tube walls, causing emissions of secondary electrons. An electric field in the tube, due to 
Table 2 Characteristics of the DES and MIS MCP detectors and assembly

\begin{tabular}{lll}
\hline MCP parameter & DES value & DIS value \\
\hline Outside radius & $44 \mathrm{~mm}$ & $56 \mathrm{~mm}$ \\
Inside radius & $\mathrm{n} / \mathrm{a}$ & $37 \mathrm{~mm}$ \\
Thickness & $1.5 \mathrm{~mm}$ & $1.0 \mathrm{~mm}$ \\
Pore diameter & $25 \mu \mathrm{m}$ & $25 \mu \mathrm{m}$ \\
Pore center-to-center spacing & $32 \mu \mathrm{m}$ & $32 \mu \mathrm{m}$ \\
Bias angle & $8^{\circ}$ & $8^{\circ}$ \\
Plate resistance & $18.2-36.4 \mathrm{M} \Omega$ & $16-30 \mathrm{M} \Omega$ \\
Plate-to-plate resistance matching & $10 \%$ & $10 \%$ \\
Grid transmission & $90 \%$ & $90 \%$ \\
MCP-to-grid spacing & $2.7 \mathrm{~mm}$ & $2.7 \mathrm{~mm}$ \\
Inter-MCP spacing & $51 \mu \mathrm{m}$ & $51 \mu \mathrm{m}$ \\
MCP-to-anode spacing & $2.0 \mathrm{~mm}$ & $2.0 \mathrm{~mm}$ \\
\hline
\end{tabular}

the high voltage applied, accelerates the secondary electrons down the tube. Subsequent impacts of these secondary electrons upon the tube walls create additional secondary electrons, yielding an avalanche of electrons exiting the bottom of the MCP stack. At appropriate operating voltages, the electron charge avalanche delivers several million electrons over roughly a nano-second. The chevron configuration-consisting of two MCPs and so-named for the V-shape the pores in the two plates form when viewed in cross section-supplies the sufficient gain while also suppressing ion feedback.

The DES and DIS MCPs and assembly have characteristics as listed in Table 2. They are composed of Photonis' Long Lifetime Material with Nichrome (80/20) electrodes. The relatively low resistance of these MCPs maximizes the strip current at a given voltage, in turn maximizing the count rate possible before the second stage MCP begins to saturate. The DES MCPs are semi-circular in shape, with only the outer semi-annular region located directly under the $180^{\circ}$ ESA exit aperture. The DIS MCPs are annular in shape and extend to an arc of $220^{\circ}$ providing uniformity of the electrostatic environment over the $180^{\circ}$ of active detector range (Fig. 13). The annular shape conforms to the annular ESA aperture, yielding a relatively high ratio between the area illuminated by signal ions to the total area of the MCP, which enhances its ability to respond linearly at high event rates. The DIS chevron stack resistances range from $32.7 \mathrm{M} \Omega$ to $47.3 \mathrm{M} \Omega$ and average $41.4 \mathrm{M} \Omega$.

In the case of DES, high positive voltage is applied to the bottom of the MCP stack and the anode. For DIS, high negative voltage is applied to the top of the MCP stack and the grid, while the anode is held at signal ground. In both cases, the anode is operated at a voltage more positive than the bottom of the MCP stack in order to enhance collection of the MCP-generated electrons.

MCP Processing, Selection, and Life Tests The DES MCPs were pre-conditioned by extracting signal charge to a level of $0.1 \mathrm{C} / \mathrm{cm}^{2}$ in a bake-and-scrub facility at GSFC. A UV source and $\mathrm{Ni}^{63}$ source were used for the burn-in process with the bulk of the charge being extracted by UV illumination. Periodically the UV source was interrupted, leaving only the $\mathrm{Ni}^{63}$ source to excite the MCPs at lower rates and allowing a measurement of the electron pulse height distributions. The MCP stack gain was tracked throughout, measured as the ratio of the first and zeroth integral moments of the pulse height distribution, and width, estimated as the ratio of the FWHM of the pulse height distributions divided by the gain. There was no separate bake-out during the conditioning process. 
Fig. 13 DIS MCP mechanical characteristics
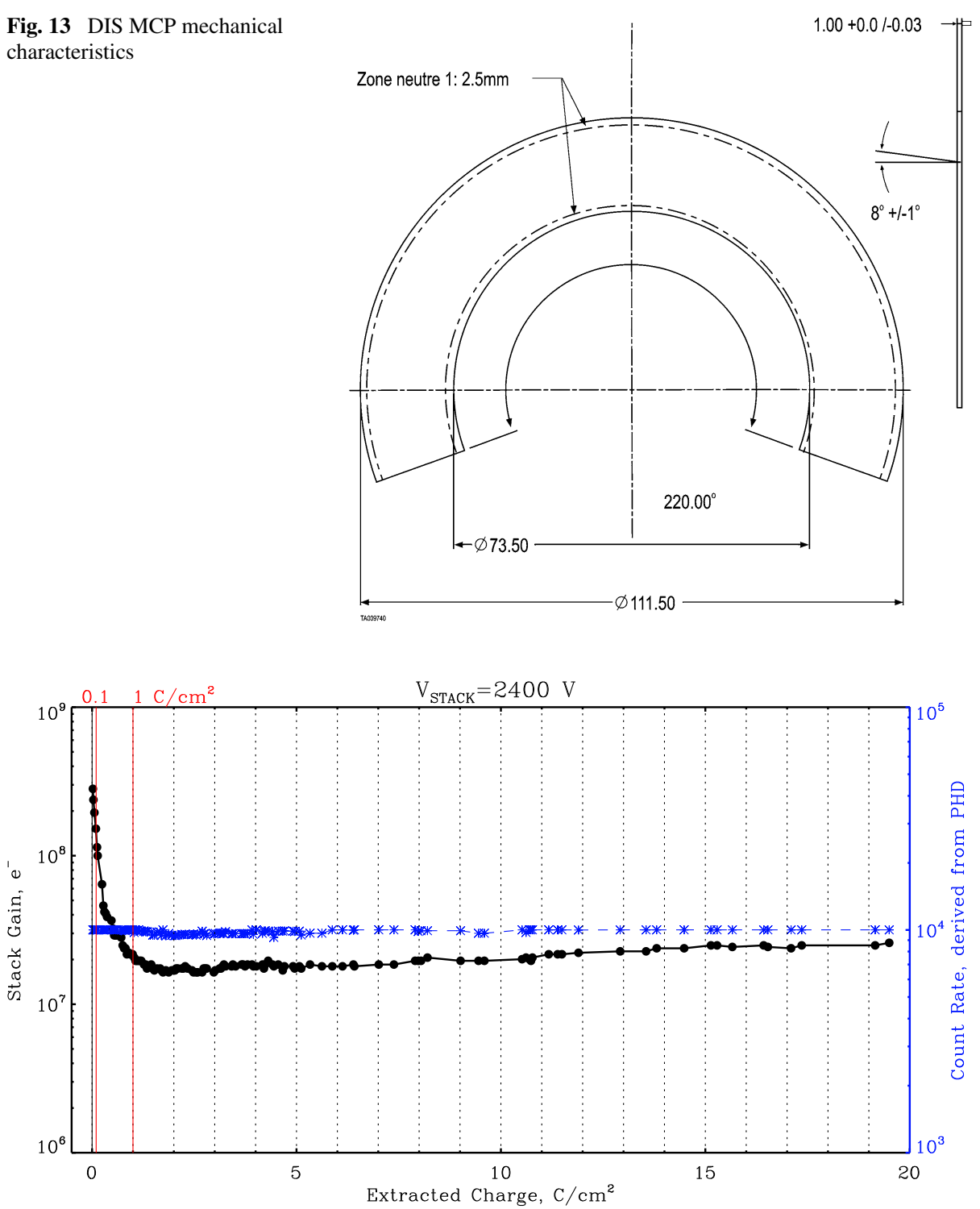

Fig. 14 MCP gain (left axis, black) and count rate (right axis, blue) measured during life test are plotted versus the estimated extracted charge. The vertical red lines indicate the nominal charge/area removed by the normal DES MCP scrub process (near $0.1 \mathrm{C} / \mathrm{cm}^{2}$ ) and the charge/area removed when stable gain is achieved (near $1.0 \mathrm{C} / \mathrm{cm}^{2}$ )

A MCP life test, extracting nearly $20 \mathrm{C} / \mathrm{cm}^{2}$, was performed on two spare stacks DES flight MCPs. Figure 14 shows a steep degradation in gain over the first $\mathrm{C} / \mathrm{cm}^{2}$ of charge emission but no significant degradation in gain (black, left axis) and count rate (blue, right axis) over the duration of the test (results were similar for the two stacks). The count rate, which is a good proxy for the stack efficiency, was derived as the zeroth integral moment of the pulse height distribution performed between limits and geometry that did not change 
Fig. 15 Test board used to characterize DIS chevron MCP stacks. The assembly is shown with the MCPs mounted and without the 3-holed test mask

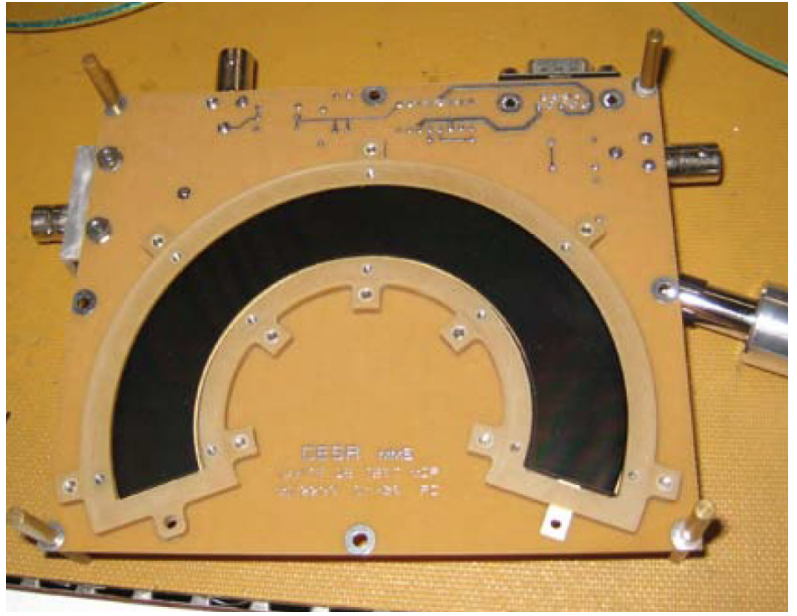

over the life of the test. Thus the pre-conditioning procedure of $0.1 \mathrm{C} / \mathrm{cm}^{2}$ extracted charge was only partially helpful in bringing the DES MCPs toward their stable gain values in flight. Based on this, the $V_{M C P}$ will be adjusted at a rate up to $50 \mathrm{~V} / \mathrm{month}$ during the first year of the mission after which the gain is expected to stabilize. By the end of the MMS prime mission, the DES MCP stacks should emit fewer than $5 \mathrm{C} / \mathrm{cm}^{2}$.

The DIS MCP pairs were preconditioned by IRAP. Prior to performing electrical testing, a proprietary liquid cleaning process was applied to the MCPs. This is the same process used for previous missions, including Interball Electron Experiment (Sauvaud et al. 1997), the Solar Wind Electron Analyzer on STEREO (Sauvaud et al. 2007), and the Cluster Ion Spectrometry experiment on Cluster (Rème et al. 1997). For testing, the DIS MCPs were mounted on a board equipped with an Amptek A203 amplifier and supporting circuitry. A mask was set $2 \mathrm{~cm}$ above the chevron input face. Three $4 \mathrm{~mm}$ diameter holes were made in this cover, one over the center of the $220^{\circ}$ annular MCP and two others at $90^{\circ}$ to either side, near the ends of the annular segment. A photograph of this test object with the mask removed is shown in Fig. 15. The stack's I/V characteristics, at the locations of all three of the mask holes, were recorded during high voltage ramping and pulse height distributions and plateau curves were acquired at several stack voltages between 2350 and $-2700 \mathrm{~V}$ under exposure to a beam of $700 \mathrm{eV}$ ions. A representative set of these pulse height distributions is shown in Fig. 16 and a typical plateau curve is presented in Fig. 17. A lifetime test for the DIS MCPs was deferred to early in Phase E.

DIS MCP efficiency is not expected to degrade with increasing energy/charge as strongly as for DES. Also, the input faces of the DIS MCP detector assemblies are biased negative of $-2 \mathrm{kV}$ to ensure all ions incident upon the DIS MCP detectors have energies/charge greater than or equal to $2 \mathrm{kV}$. Regardless, for both DES and DIS, some differential gain variation is expected within individual MCP stacks. That is, over time some FOV pixels will exhibit larger gain degradation than others in proportion to their exposure to impinging plasma populations. As this differential gain degrades, both signal loss and capacitive cross talk grows. Operational procedures are in place for monitoring MCP gain, pixel-by-pixel, adjusting $V_{M C P}$ to minimize temporal gain variation through the mission, and tracking pixellevel signal loss and crosstalk for incorporation into ground data processing tables.

Discrete Anodes For both DES and DIS, the electron charge cloud from the MCP is collected on 16 discrete anodes located just below the MCP stack, each covering $11.25^{\circ}$ on 
Fig. 16 Typical pulse height MCP testing at IRAP distributions acquired during DIS

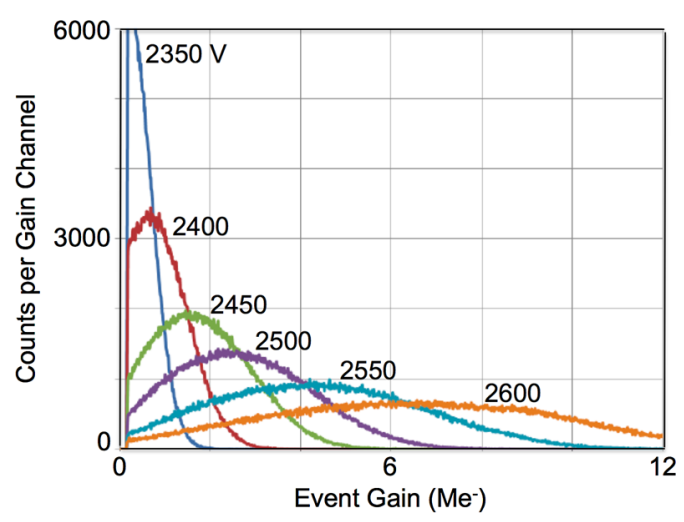

Fig. 17 A typical MCP plateau curve acquired during DIS MCP testing at IRAP

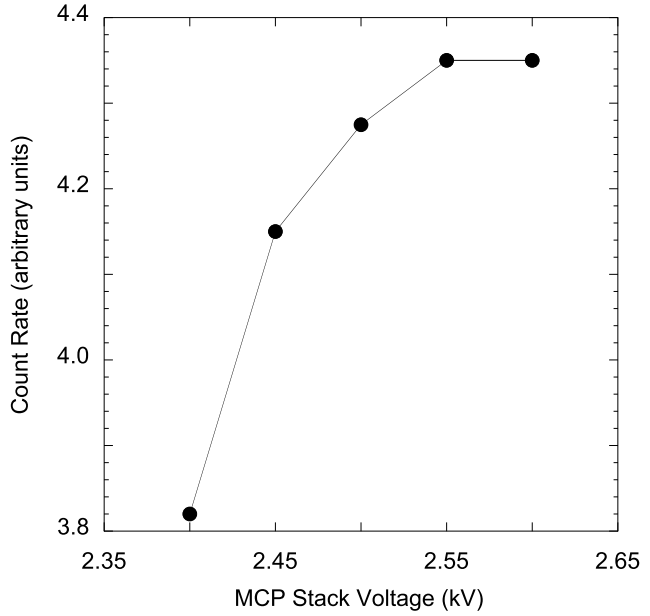

the anode board. Experimentation with the high fidelity DES ETU determined that optimal charge cloud transmission could be achieved with a $2.0 \mathrm{~mm}$ spacing between the output face of the second stage MCP and the anode along with installation of a capacitor between that output face of that MCP and ground. Figure 18a, which corresponds to gaps smaller than 0.5 $\mathrm{mm}$ exhibit voltage transients upon emission of signal charge pulses due to capacitive coupling between the anode and the MCP face, Fig. 18b shows results after increasing the gap from $0.5 \mathrm{~mm}$ to $2.0 \mathrm{~mm}$ and Fig. $18 \mathrm{c}$ shows the results after the additional of the capacitor. The addition of the capacitor substantially suppresses cross talk.

In addition, capacitive AC coupling is necessary to decouple the high voltage on the anodes from the input of the A121s. In the case of the DES anodes, the charge pulse is AC-coupled through a high voltage capacitor into the input of the A121. In DIS, as its input is at $1.2 \mathrm{~V} \mathrm{DC}$, the anodes are DC coupled to ground through high resistance and then AC coupled to the A121. In both cases, the charge pulses are converted by the A121s into voltage signals and injected into voltage pulse height discriminators.

Pre-Amplifier/Discriminator Input voltage pulses that exceed the discriminator threshold will result in the generation of countable digital output pulses from the A121, whereas input voltage pulses that do not exceed threshold will not. For FPI, this threshold is set near 


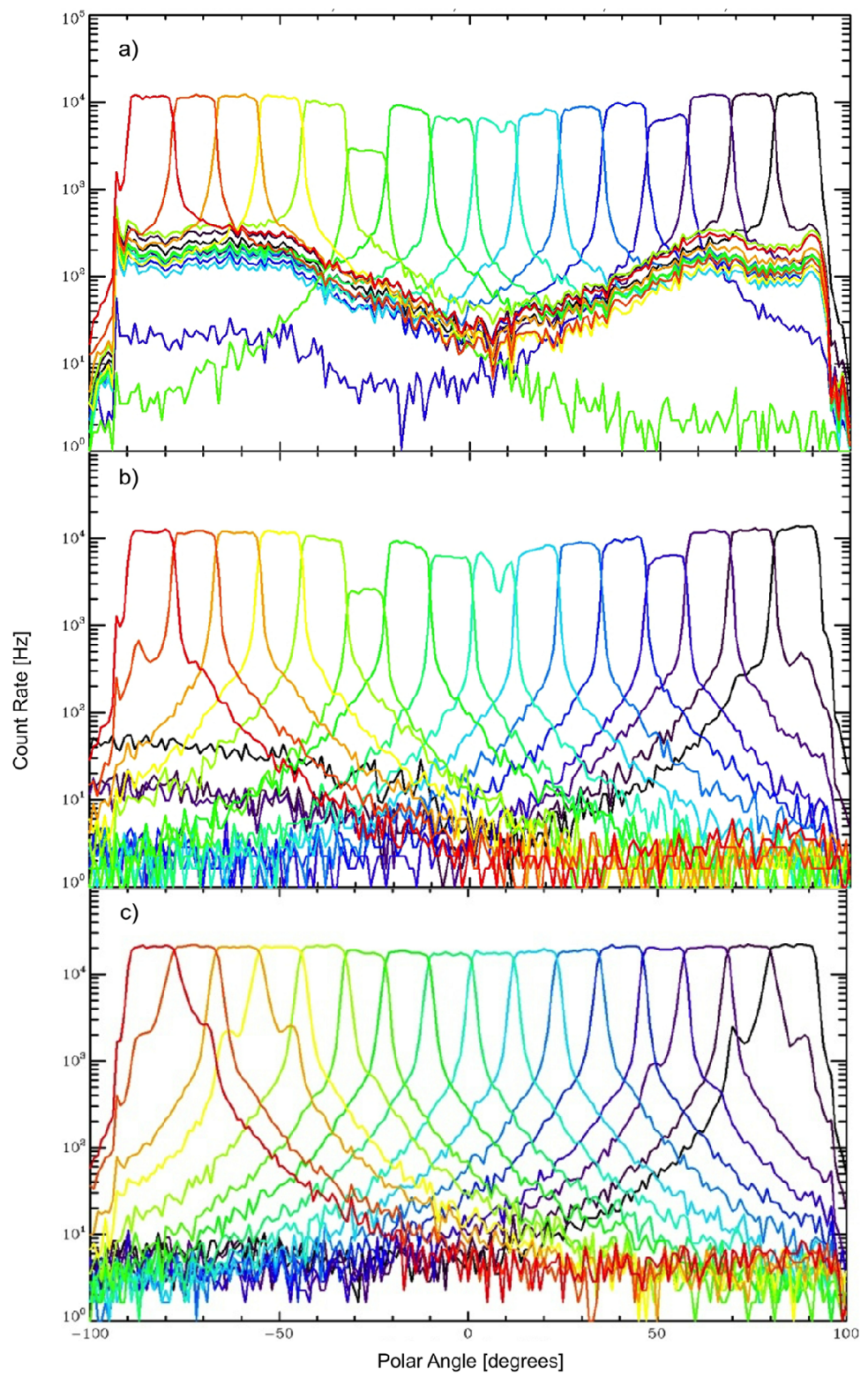

Fig. 18 Sequential improvement in DES detector performance with changes made to the detector design and discrimination threshold. (a) MCP to anode gap of $0.5 \mathrm{~mm}$ and discriminator threshold of $6.5 \times 10^{5}$ electrons/pulse. The high background shared across pixels is caused by capacitive coupling of voltage transients on the MCP output surface to the anode array. The higher discrimination threshold reduces the impact of the effect. (b) MCP to anode gap of $2 \mathrm{~mm}$ and discriminator threshold of $6.5 \times 10^{5}$ electrons/pulse. The increased gap reduces the uniform high background but still requires a large discrimination threshold. (c) MCP to anode gap of $2 \mathrm{~mm}$, MCP exit decoupled with capacitor and discriminator threshold of $2.5 \times 10^{5}$ electrons/pulse. The addition of the capacitor virtually eliminates the cross talk even at the lower discrimination threshold. This reduction in discrimination threshold levels the pixels' peak rates, owing to decreased signal loss in panel (c), as compared with those in panels (a) and (b). The artifacts seen for pixels away from the center of the polar scan are due to an anode board layout issue that was later corrected 
FM216 Injected Signal vs Threshold at Initial Ambient

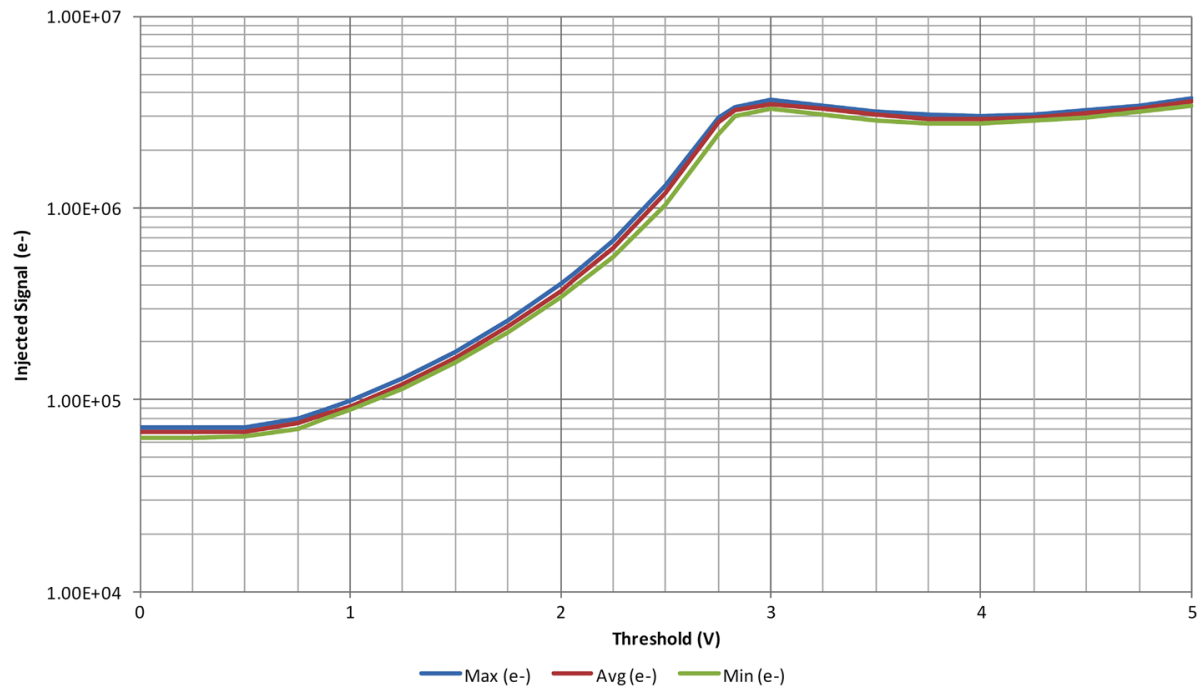

Fig. 19 Prototypical discriminator threshold is plotted versus applied voltage at A121 Pin 3

$4 \times 10^{5}$ electrons/pulse, varies slightly among detector assemblies, and is well below our typical operating gain, near $2 \times 10^{6}$ electrons per pulse (the pulse generation efficiency of these systems is typically less than unity). Significantly, the temperature dependence of the A121 threshold setting minimizes near this threshold value. It is worthwhile to note that while individual A121 discriminator thresholds are controllable by telecommand, limited Digital-to-Analog Converter (DAC) resources forced a single threshold being used for the sixteen channels in each DxS detector system.

The A121 threshold control is a nonlinear function of the voltage applied to its Pin 3 (Vpin-3). Figure 19 shows the typical behavior of this dependence, which varies from $7 \times$ $10^{4}$ electrons at $0 \mathrm{~V}$ to $4 \times 10^{6}$ electrons near $3 \mathrm{~V}$. Above $2.75 \mathrm{~V}$, the threshold increase flattens, rendering threshold control less effective above $3 \times 10^{6}$ electrons/pulse. The actual dependence varies from device to device. This feature would impact the use of threshold control to measure MCP stack gain, for gains above $\sim 3 \times 10^{6}$ electrons per pulse.

Extensive threshold characterization data for each device guided separation of the flight devices into groups of 16 with similar threshold-versus-Vpin-3 characteristics. This device matching significantly reduces pixel-to-pixel threshold variability and a uniformity of $\sim 10 \%$ was achieved within each DxS.

Photographs of the front and backside of typical DES and DIS anode boards are shown in Figs. 20 and 21. On the front side of the DES anode board are seen the 16 discrete anodes, 10 of 16 Amptek A121 devices and eight of sixteen high voltage capacitors that provide isolation between the anode and the low voltage components on the board, most importantly for the A121s. On the DES anode board backside are the other eight high voltage capacitors, six A121s and an array of resistors that enable drainage of the deposited electrons back into the high voltage circuit. Also visible on both the front side and backside of the anode board are protective diodes and other low voltage circuitry required for A121 functionality.

In the case of DIS, the individual anodes are separated by grounded shield traces reducing capacitive crosstalk among the anodes. On the back side, the array of 16 A121 pre-amplifiers are visible, along with a grounded shield frame that makes contact with the 

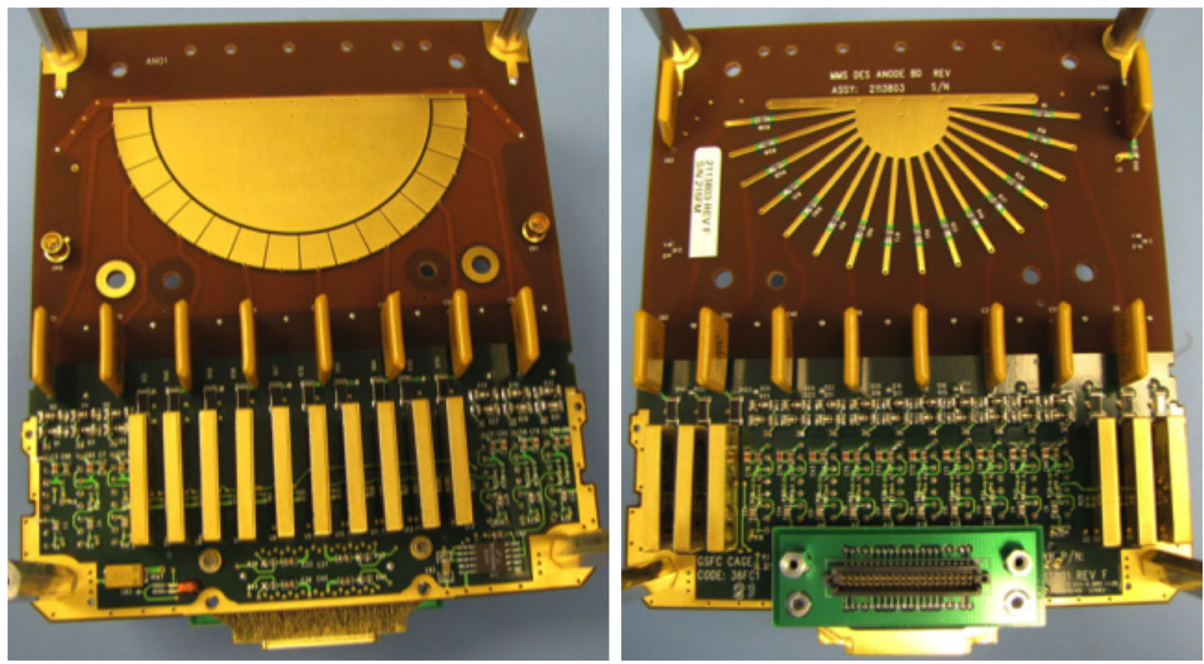

Fig. 20 Photographs of the DES anode board, front (left) and rear (right)
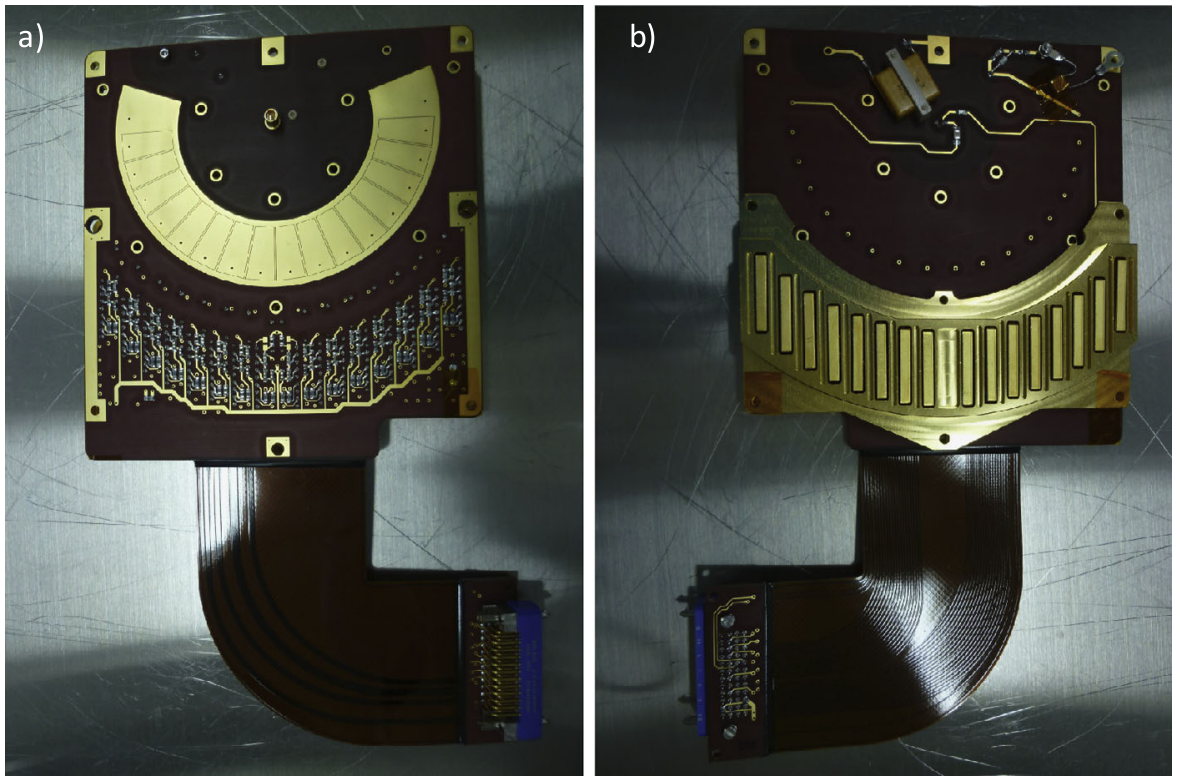

Fig. 21 Photographs of the DIS anode board and flex cable assembly: (a) front and (b) rear

amplifiers through spring clips located on either side of each A121 device. This grounded shield frame prevents voltage transients on the device case, which is otherwise connected to ground through two welded pins. This enhanced grounding ensures that voltage transients on the case do not cause spurious signals at the device inputs. The same type of grounding is used in DES.

The A121 hybrid charge sensitive amplifier/discriminator circuit used provides a dead time independent of recent pulse rate history that is adjustable. For DES, an onboard resis- 
tor provides this adjustment yielding a dead time of $100 \mathrm{~ns}$ at a periodic repetition rate of $10 \mathrm{MHz}$, which is the fastest the A121 stably supports.

\subsubsection{Electronics Box}

A single electronic box services both sensors in a DxS unit. The electronics box was implemented with its three components - the High Voltage Power Supply (HVPS), the Command and Data Handling (C\&DH) card and the Low Voltage Power Converter (LVPC) - in separate boxes, for isolation and ease of test and assembly.

High Voltage Power Supply (HVPS) The DES and DIS HVPS are similar and yet have important differences. In both cases, the HVPS contains three separate high voltage supplies co-located in the same enclosure. Two of these supplies provide separate and independent static high voltage outputs for the two DxS MCP/anode assemblies, positive (up to $+3.6 \mathrm{kV}$ ) for DES and negative (down to $-3.0 \mathrm{kV}$ ) for DIS. The third is a high-speed high voltage stepping supply with three independently controllable positive polarity fast stepping outputs to provide the ESA and deflector voltages. All are controlled by signals from the DxS $\mathrm{C} \& \mathrm{DH}$.

For the DES MCP high voltage supplies, individual taps are taken off the HVPS to provide voltage biases for the MCP/anode assemblies at five distinct points: (1) the shield grid located above the MCP input tying this to high voltage ground, (2) the MCP input face biased at 1/12th the commanded anode voltage, (3) the electrically common 1st stage MCP output face and 2nd stage MCP input face biased at 6/12th the commanded anode voltage, (4) the 2nd stage output face biased at 11/12th the commanded anode voltage, and (5) the anode itself biased at the full commanded anode voltage. This is illustrated in the schematic diagram provided in Fig. 11. As always, the most electrically positive element of the detection system is the anode itself, operated at positive high voltage in the DES case. The DES MCP high voltage supply is capable of sourcing $150 \mu \mathrm{A}$ of current.

For DIS, the MCP bias supplies have two output voltage lines, including a grounded anode bias line and a negative high voltage detector bias line connected both to the first stage MCP input face and the grid that sits directly above it. Most of this negative high voltage is dropped resistively across the chevron stack. This configuration is illustrated in the schematic diagram provided in Fig. 12 and utilizes resistive division to provide the voltages across the MCPs. Care was taken to match the MCP resistances for each chevron stack, the mean value of the resistance difference is $3 \%$ with the largest difference being $10.2 \%$. The rear face of the second stage MCP is biased with a Zener diode at -110 volts. The anode, again the most electrically positive element of the detection system, is operated at ground potential through high resistance. This minimizes charge cloud spreading between the MCP and the anodes and enhances electron collection by the anodes. The DIS MCP/anode supplies are capable of sourcing up to $100 \mu \mathrm{A}$ of current as required by the relatively low resistance of the DIS MCPs.

The third high voltage supply in each HVPS is a high-speed stepping supply with three independently controllable fast stepping outputs with 12-bit value control exercised by the C\&DH. All three stepping outputs are supplied by a common $+5.5 \mathrm{kV}$ bulk supply that can be turned on and off by the C\&DH board. One of the outputs is used to control the voltage of the ESAs in both dual sensor heads. The ESA output is positive in DES and negative in DIS to provide the energy selection of electrons and ions, respectively. The other two outputs are positive and each control one of the two deflector plates, DEF0 and DEF1, in each sensor head. This supply is shared between both sensors of a DxS. 


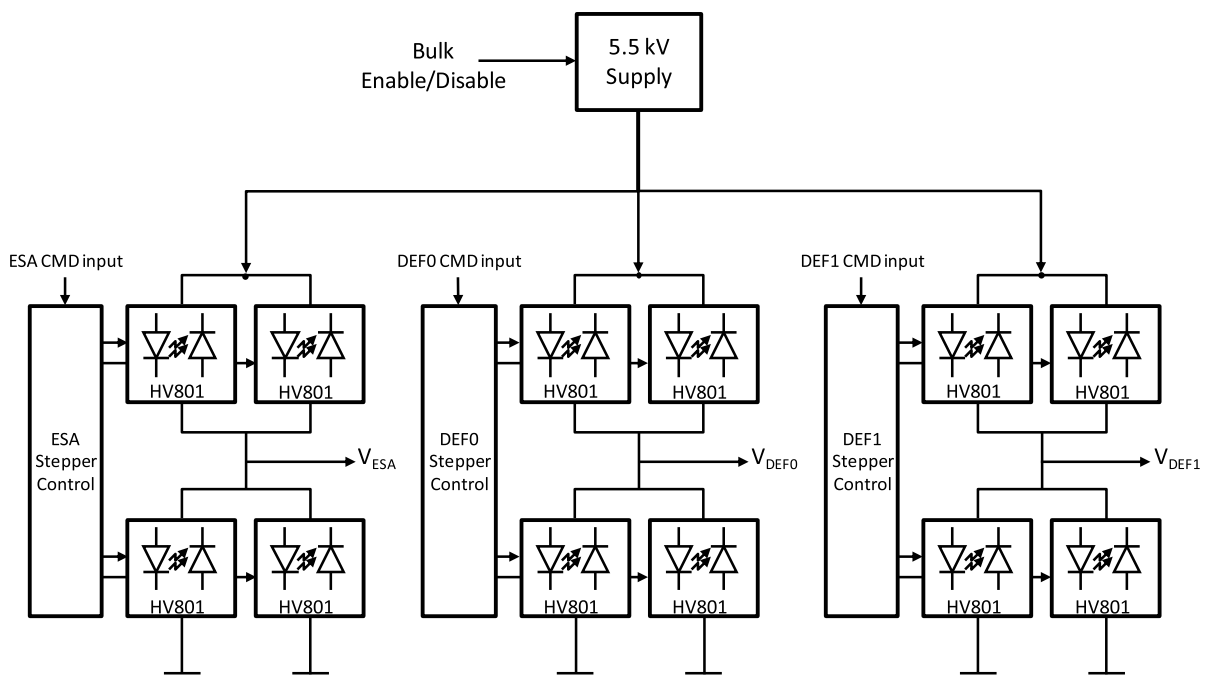

Fig. 22 Simplified block diagram of the ESA/Deflection high voltage stepping supplies of the DES HVP

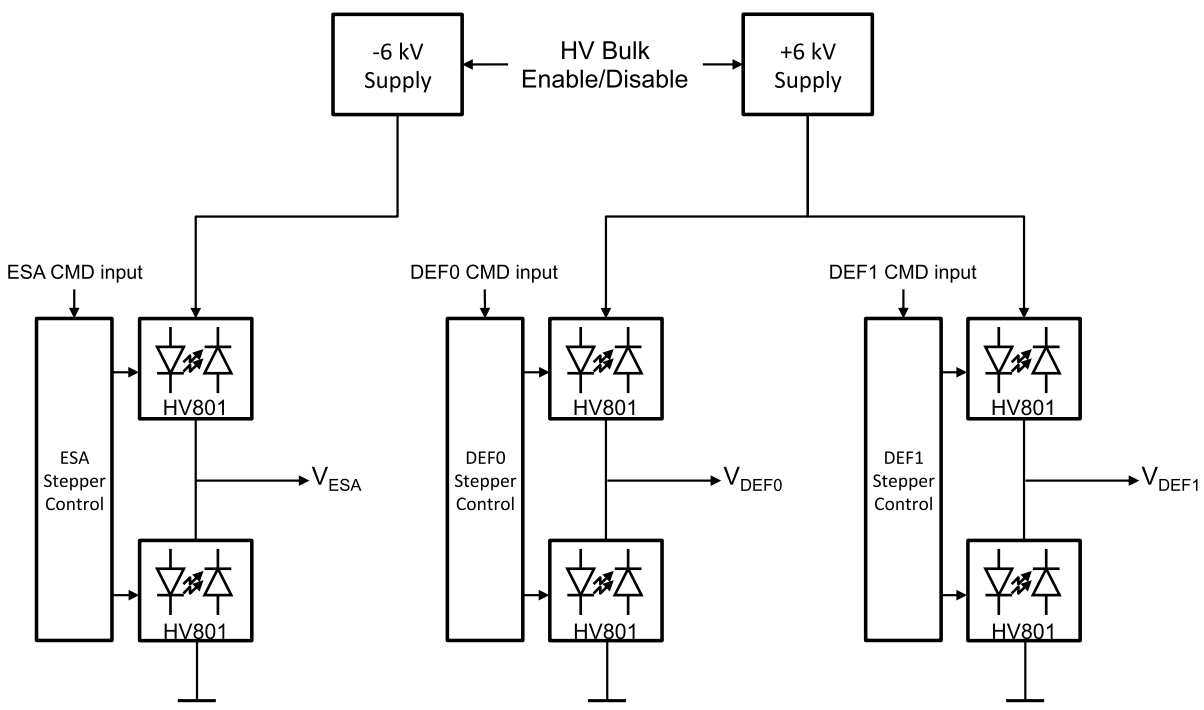

Fig. 23 Simplified block diagram of the ESA/Deflection high voltage stepping supplies of the DIS HVPS

The stepping supplies are both dual range, with a low range extending from a fraction of a Volt to $50 \mathrm{~V}(60 \mathrm{~V})$ for DES (DIS) and an overlapping high range that extends from $\sim 1 \mathrm{~V}$ to $5 \mathrm{kV}(5.8 \mathrm{kV})$ for DES (DIS).

Simplified block diagrams of the DES and DIS high voltage stepping supplies are shown in Figs. 22 and 23. The stepping outputs (deflectors and ESA) are supplied by bulk supply outputs as appropriate. The bulk outputs are based on one converter that can be turned on and off by the C\&DH board. The voltage reaching the stepper outputs is regulated by push-pull 
Fig. 24 DxS low voltage power converter-power interfaces

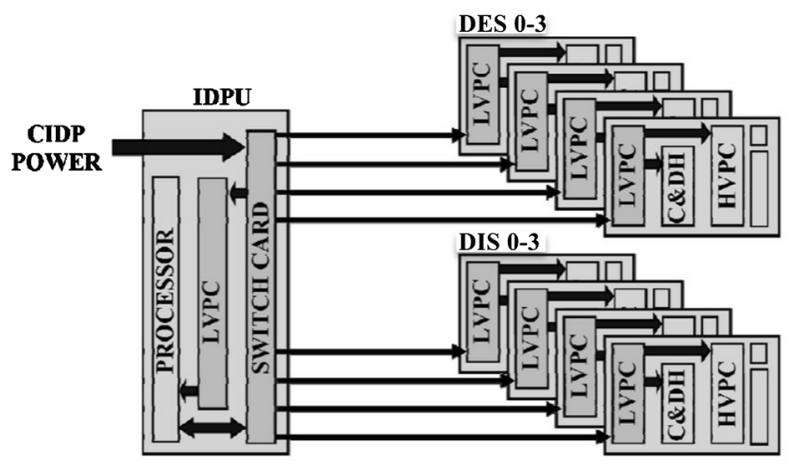

Amptek HV801 opto-coupler networks with feedback control loops in the HVPS driving to the voltage commanded by the C\&DH board.

For each DIS (DES) output stepping circuit, the opto-coupler network consists of one (two in parallel) upper opto-couplers and one (two in parallel) lower opto-couplers. In this configuration, the voltage across the lower opto-coupler(s) is always equal to the output voltage and the voltage across the upper opto-coupler(s) is always equal to the relevant bulk output value minus the output voltage for each deflector. This push-pull configuration is used for power efficiency and to achieve the required fast stepping capabilities. For DES, the two opto-couplers in parallel at each location are used because the fast stepping requires a higher current C\&DH Board.

Command and Data Handling (C\&DH) Card The C\&DH board collects and counts the digital pulses from the preamplifier/discriminators on the anode boards of both sensor heads and integrates those counts during a configurable integration time. It further controls the HVPS and thereby the ESA and deflectors as well as the MCP and the single detection threshold of the preamplifier/discriminators. Finally, the C\&DH measures a variety of low voltage, high voltage and temperature housekeeping states from the entire dual spectrometer. The C\&DH boards in the eight DxS on a single spacecraft communicate with and are controlled by the FPI IDPU, one per spacecraft.

Low Voltage Power Converter (LVPC) The LVPCs are functionally identical and provide the bias voltages for the C\&DH board, the HVPS and the anode board. It supplies these voltages by conversion of the instrument bus-voltage received through two diodeORed $+32 \mathrm{~V}$ bus power feeds from the MMS CIDP, routed through the Spectrometer Power Switch Card (SPSC) of the IDPU, as illustrated in Fig. 24. Each LVPC provides six ground isolated, tightly regulated, low voltage outputs. These consist of $\pm 5.5 \mathrm{~V},+3.3 \mathrm{~V},+2.5 \mathrm{~V}$ (not used in DIS) as required by the C\&DH and the detection system, and the $\pm 12 \mathrm{~V}$ required by the HVPS. The four low power $\mathrm{C} \& \mathrm{DH}$ voltages are derived from a common multiple secondary, pulse width modulated, voltage mode controlled bias network, which provides the required ground isolation and pre-regulation functions. The pre-regulated secondaries are followed by discrete linear regulator networks, which provide $\pm 1 \%$ line/load regulation and over-current protection. The higher power HVPS $\pm 12 \mathrm{~V}$ outputs are derived from two independent pulse width modulated and current-mode controlled forward power stages. The HVPS loads consist of the superposition of a DC level current with a series of variable-frequency step functions with magnitudes that reflect the sensor stepper programs. The $\pm 12 \mathrm{~V}$ control loop gains are tailored for these dynamic load conditions. The LVPC 
provides differential mode and common mode filtering on both primary and secondary sides of the $200 \mathrm{KHz}$ switching converter stages. In addition, the LVPC includes an active low frequency filter on the primary power interface to curtail very low frequency sweep ripple currents from being reflected back out to the spacecraft power bus. Additional features within the LVPC include input bus in-rush current limiting, input bus under/over voltage protection, and $\pm 12 \mathrm{~V}$ output inhibit control.

\subsubsection{Implementation and Assembly}

The DxS are implemented in modular fashion and while quite similar, each has important differences. The main parts are the two sensor heads and the electronic box, all bolted to an interface plate and interconnected with cabling. In the case of DES, shielded wires from the stepping supply of the HVPS are connected to a terminal block located between the two sensor heads. The other side of the terminal block connects to the ESAs and deflector plates inside the sensor heads, also with shielded high voltage wires. In the case of DIS, there is no terminal block. Rather, the stepper high voltage cables connect the HVPS directly to their destinations at the ESA and deflector electrodes. Additionally, MCP high voltage wires run from the HVPS and enter into the front of the sensor head to be connected to the MCP and the anode board. All high voltage wires are covered with grounded shields to reduce EMI from the high voltage supply inside the detection system. Finally, two flex cables are used to connect the two anode boards with the C\&DH board. Flex cables with ground embedded signal lines are used to enable good transmission of the output pulses from the A121s on the anode board and to shield all data and command lines from one another to minimize crosstalk to the detection system inputs and between the detection system outputs. Careful attention to control the amount of high voltage noise and potential for crosstalk in the detection systems significantly improved detection performance.

For DES, after optics installation, the sensor heads were installed onto the interface plate. The MCP stacks were tested and pre-conditioned and the anode boards tested and calibrated. The MCP stacks were then assembled into their holders and installed on the anode boards that were installed into the sensor heads. In the case of DIS, anode boards were fabricated, populated and tested in Japan before sending them to IRAP in France. At IRAP, MCP stacks were installed onto the anode boards yielding completed detector assemblies. After installation testing and calibration at IRAP these detector assemblies were sent back to Japan, where they were installed into the sensor heads.

After connecting the high voltage cables to the detector assemblies, the flex cables between the anode boards and the C\&DH boards were installed. Covers were installed over the sensor heads and over the bridging between the sensor heads and the electronics box. Side covers were installed to close out the cabling space between the electronics box, the sensor heads and the anode boards. Finally for each DxS unit, external heaters, thermistors, and thermostats as well as a purge system for the sensor heads were installed. After assembly, each DxS was functionally and environmentally tested and fully calibrated using a charged particle beam under clean high vacuum.

\subsubsection{Functionality of the System}

Here we describe the basic DxS function, with special emphasis on the Fast Survey mode of operation when MMS is in the scientific region of interest. Each DxS is a pair of particle spectrometers designed to measure differential directional flux in a selected energy 


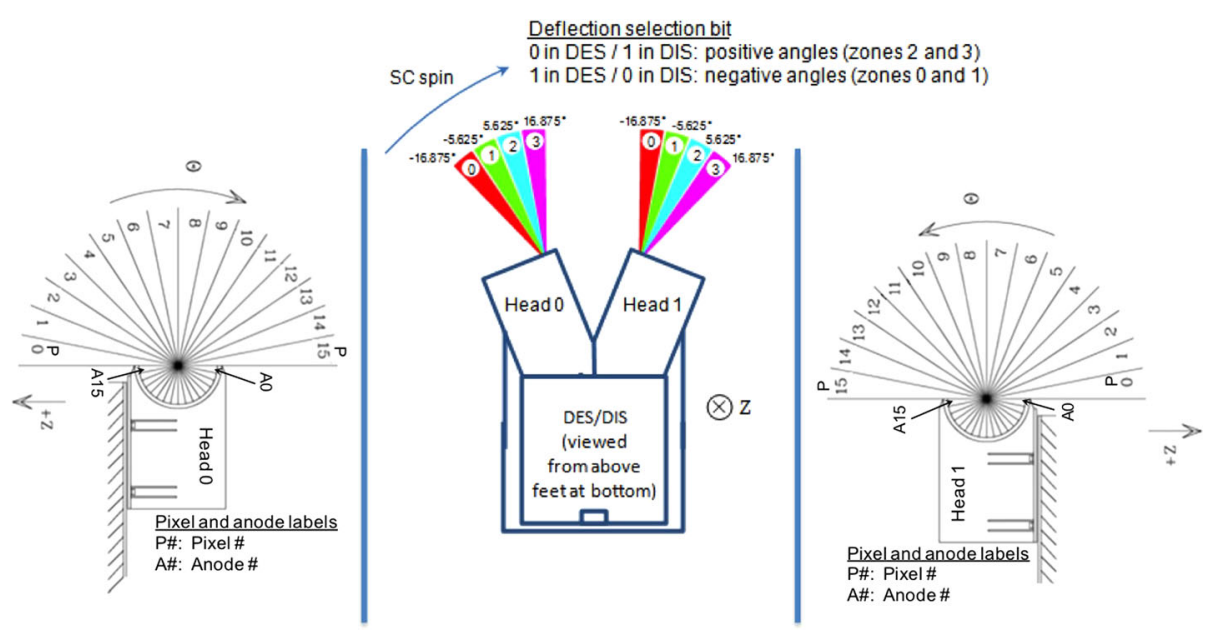

Fig. 25 Definitions of polar angle pixels, anodes, azimuth zones (colored) and look angles for each DxS. The spacecraft $+Z$ axis is consistently indicated

range and from selected directions. Particles enter the sensor head aperture through a double grid and pass through deflector plates to the ESA; together, these components constitute the optics of the spectrometer. The deflector plate potential selects the azimuth angle look direction. The top hat ESA analyzer selects plasma particles that fall within its energy resolution, $d E / E$, centered at an energy equal to the product of the applied voltage and the ESA Analyzer Constant $\left(k_{\epsilon}\right)$. Particles of higher energy fly wide and hit the outer ESA wall and those of lower energy are driven into the inner ESA wall. Particles that pass through the ESA without impacting a wall are detected and counted.

The combination of MCP gain (typical number of electrons delivered by an event avalanche) and A121 threshold setting constitutes the operating point for this detector assembly. The detector operating point has a significant impact on detector system performance, affecting both signal loss from pulses that do not exceed threshold and system cross talk owing to capacitive coupling among anodes. Selection and maintenance of a detector assembly operating point therefore represents an important element of both ground and flight calibration (see Gliese et al. 2015).

For every 16-element discrete anode array the same anode numbering convention (0-15) is used. Since the two sensors in a DxS are assembled back-to-back, the anode arrays are flipped with respect to one another in the spacecraft frame of reference. For example, a plasma particle moving toward the spacecraft from $+Z$ (in the spacecraft reference frame its vector velocity points in the $-Z$ direction) would detected by DxS Sensor 0 in anode 0 , but be detected in Sensor 1 in anode 15. Therefore an FPI convention is adopted such that 'anodes' and 'pixels' are defined separately. The anode array is defined in the frame of reference of the detector system, while the pixel array is defined in the frame of reference of the DxS which, when mounted on the spacecraft, is identical to the spacecraft frame, to within an azimuth phase shift. In the example above, the particle would be detected in pixel 0 in either Sensor 0 or Sensor 1.

These conventions are illustrated in Fig. 25, where definitions of DxS pixels (labeled "P") in spacecraft polar coordinates and their relationships to the anode (labeled "A") arrays of each sensor head are provided. Figure 25 has three elements: left, center and right. On the left and right, the pixel arrays associated with DxS sensors 0 and 1 are shown. The sensors 
are shown mounted on the MMS instrument deck. In flight, the $+Z$-axes of all four MMS spacecraft are directed northward, perpendicular to the $28^{\circ}$ inclination orbit plane. In the spacecraft spherical coordinate system, the relation between spherical polar angle and the pixel viewing that angle is nominally (to within calibrated differences) identical for all DxS sensors and is given by:

$$
\theta=5.625+11.25 \times p
$$

where $\theta$ is the spherical polar angle viewed $\left(0-180^{\circ}\right)$, and $p$ is the pixel number $(0-15)$. It is important to understand that this is the angle viewed by the pixel, not the polar angle associated with the detected particles' vector velocity. The velocity direction of a detected particle is opposite to the direction viewed by the detecting pixel.

The center panel of Fig. 25 illustrates a DxS and its eight azimuthal FOVs (Zones 0-3 for each of two heads) as seen when viewing along the spacecraft $+Z$ direction. Each DxS sensor deflects its instantaneous spacecraft azimuth FOV center from $-16.875^{\circ}$ to $+16.875^{\circ}$ in four zones centered on $-16.875^{\circ},-5.625^{\circ},+5.625^{\circ}$ and $+16.875^{\circ}$. The azimuth look angle and the zone numbers are defined to increase in the direction of spacecraft spin. However, owing to the "umbrella" shape of the deflected fan of pixels, the pixels from adjacent sensors tend to interleave with each other as polar angle progresses toward the poles.

The deflection outputs are strictly positive in polarity so as not to cause energetic secondary or photoelectron emission from the DxS deflection electrodes, which have direct view to the external environment through grounded aperture screens. Each deflection output controls a single deflector plate in each DxS sensor head. In both sensors of a DxS, one of the two deflection outputs is used to achieve positive angular deflection, looking toward increasing spin phase, and the other deflection output is used to achieve negative angular deflection, looking toward decreasing spin phase.

To accomplish measurements at all 32 required energies and 4 azimuth zones with the required temporal resolution of $30 \mathrm{~ms}$ (DES) or $150 \mathrm{~ms}$ (DIS), the DxS ESA voltage and deflector voltages are stepped rapidly as illustrated in Fig. 26, where nominal maximum and minimum energy target centers are shown (actuals for DES or DIS may be slightly different). In $7.5 \mathrm{~ms}$ (DES) or $37.5 \mathrm{~ms}$ (DIS), the HVPS ESA stepper output is stepped through a progression of 32 logarithmically spaced voltage steps (say the even steps, $0,2, \ldots, 60,62$ of a 64-step sequence) sampling electrons (DES) or ions (DIS) from the low to the high end of the required $E / q$ range. Simultaneously and in synchronization with the ESA stepping, the HVPS DEF1/0 (DES/DIS) stepper output is stepped through 32 voltage steps of its own, as required to maintain a constant Zone- 0 deflection centered near $-16.875^{\circ}$ for both sensor heads at each of the 32 energies/charge sampled in the ESA sequence. This is achieved by stepping just the HVPS DEF1/0 (DES/DIS) stepper output. The HVPS DEF0/1 (DES/DIS) stepper output is held near ground through this portion of the sequence. The DEF1 stepper output is wired to the lower deflection plate in sensor- 0 and to the upper deflection plate in sensor-1 whereas the DEF0 stepper output is wired to the upper deflection plate in sensor-0 and to the lower deflection plate in sensor-1 in both DES and DIS.

This process first provides samples in both DxS sensors at 32 energies/charge within azimuth angle Zone- 0 , centered at $-16.875^{\circ}$. The process is then repeated, hitting the same (even) energy/charge steps on a downward ESA stepper sweep, again using only the DEF1/0 (DES/DIS) stepper output to sample azimuth angles within Zone-1, near $-5.625^{\circ}$. Whereas the target voltages on this downward ESA stepper sequence are identical to those on the upward ESA sequence just executed, the target voltages on the corresponding Zone-1 DEF1/0 (DES/DIS) stepper sequence are smaller at any $E / q$ by approximately a factor of 3 , than 


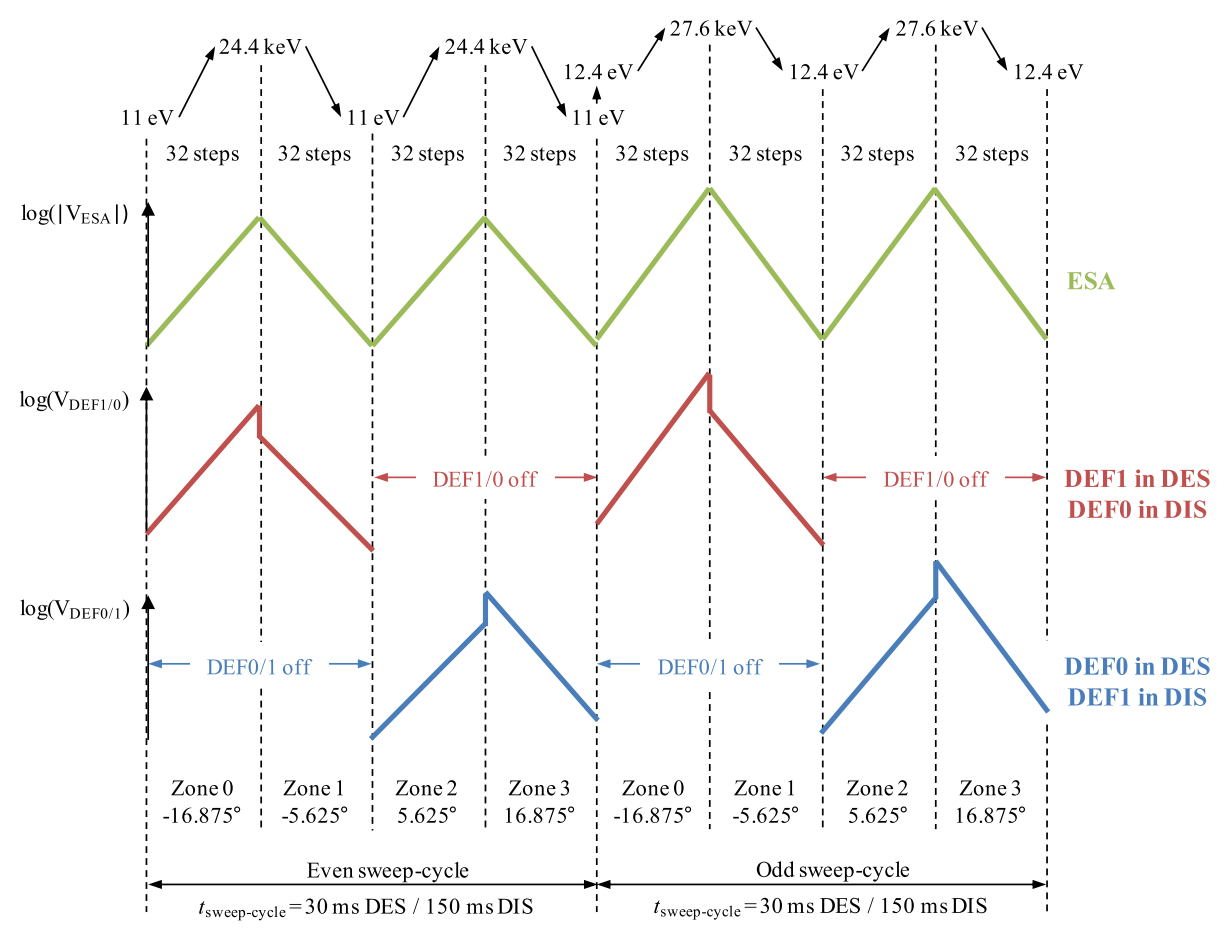

Fig. 26 Control of the ESA and deflector plates in the DxS to achieve measurements across all required electron energies and azimuth look angles (zones). Note that the bounding energies shown at top represent band pass centers such that the lowermost and uppermost band pass edges correspond to $10 \mathrm{eV}$ and $30 \mathrm{keV}$ (the DES example with $d E / E \sim 0.17$ is used for illustration)

those in the previous Zone- 0 sequence, corresponding to the 3 times smaller deflection required for Zone-1 $\left(-5.625^{\circ}\right)$ compared to that required for Zone- $0\left(-16.875^{\circ}\right)$. Again, while sampling Zone-1, the HVPS DEF0/1 (DES/DIS) stepper output is held near ground.

Having sampled Zone- 0 and Zone- 1 at all 32 even energy steps, a similar up/down even $E / q$ step sequence is executed to sample fluxes within Zone-2 $\left(+5.625^{\circ}\right.$, upward $E / q$ sweep) and then within Zone-3 $\left(+16.875^{\circ}\right.$, downward $E / q$ sweep). For Zones 2 and 3, the HVPS DEF0/1 (DES/DIS) stepper is activated, stepping synchronously with the HVPS ESA stepper while the HVPS DEF1/0 (DES/DIS) stepper output is held near ground. The four energy sweeps described above (upward, even $E / q$, Zone-0; downward, even $E / q$, Zone-1; upward, even $E / q$, Zone-2; downward, even $E / q$, Zone-3) require 128 distinct stepper states - and $30 \mathrm{~ms}$ (DES) or $150 \mathrm{~ms}$ (DIS) to execute-providing the required DxS time resolution but at one-half the $E / q$ sampling density that our 64-step logarithmic sweep provides. Therefore, the entire 128-step sequence is then repeated, utilizing the odd $E / q$ steps from the 64-step sweep. Thus the complete DxS energy angle sequence is comprised of 256 states that are repeatedly executed every $60 \mathrm{~ms}$ for DES and $300 \mathrm{~ms}$ for DIS.

DxS deflector voltages are always positive and only one deflector in each sensor head is energized at any time, depending upon the algebraic sign of $\alpha_{c e n t e r}$. For positive values of $\alpha_{\text {center }}$ (Zones 2 and 3, looking toward increasing spacecraft spin phase), the leading deflectors (upper for sensor head 0 and lower for sensor head 1) are energized for DES through the DEF0 supply output and the trailing deflectors (lower for sensor head 0 and upper for sensor head 1) are energized in the case of DIS through the DEF1 supply output. 
For negative values of $\alpha_{\text {center }}$ (Zones 0 and 1, looking toward decreasing spacecraft spin phase), the trailing (lower for sensor head 0 and upper for sensor head 1) deflectors are energized for DES through the DEF1 supply output and the leading deflectors (upper for sensor head 0 and lower for sensor head 1) are energized in the case of DIS through the DEF0 supply output. In every case, the deflector electrode that is not energized is held at ground potential. Based on the defined 32 energy steps (up/down for first two angles then up/down for the next two angles) and the interleaved 32 energy steps (again, up/down for the first two angles then up/down for the next two angles) two stepper tables, each comprised of 128 states, are constructed containing all the required ESA, DEF0 and DEF1 voltages to be set in succession to achieve execution of the complete stepping cycle and resulting in the output voltages shown in Fig. 26. In this manner a full but somewhat coarse energy/angle array is sampled every $30 \mathrm{~ms}$ (DES) or $150 \mathrm{~ms}$ (DIS) and filled in to provide more dense energy sampling every $60 \mathrm{~ms}$ (DES) or $300 \mathrm{~ms}$ (DIS).

The average time allocated between stepper transitions is $234 \mu \mathrm{s}(30 \mathrm{~ms} / 128 \mathrm{steps})$ for DES and $1172 \mu \mathrm{s}(150 \mathrm{~ms} / 128$ steps $)$ for DIS. These durations are apportioned between HVPS settling time (both ESA and DEF) and count integration time. The count integration times for each species are the same for every step in the sequence: $195 \mu$ s for DES and $1000 \mu$ s for DIS. The settling times are apportioned across the sweeps based on detailed characterization of the dynamic behavior of each high voltage stepper supply.

Command and Data Handling The C\&DH systems are responsible for communication with the FPI IDPU, control and monitoring of the HVPS and LVPC, collection and transmission of pulse data from the detector assemblies, control of pulse detection thresholds, counting of pulses and control of pulse count integration time, control of stimulus test sources for the detection system, and collection and transmission of monitored housekeeping data.

Each C\&DH board executes commands received from the IDPU in a command-response format. The IDPU operates in one of two basic modes of operation. When the FPI suite operates in engineering, calibration, or safe modes, it issues single independent commands to the C\&DH. When the suite is operating in Fast or Slow Survey mode, the IDPU issues a three-step command and data pipelined process to each C\&DH. Upon receipt of appropriate commands from the IDPU, the C\&DH board enables the detector output pulse counters, waits for a pre-programmed period of time (integration period), disables the counters, copies the data in the counters, resets the counters, and transmits the counter data to the IDPU. During this process, it also writes command data to the stepping HVPSs, transfers data from the housekeeping system to the IDPU for the low voltages, for temperatures monitored in the detection system, HVPS and LVPC, and for the MCP voltages and currents, and sends channel selection information to the housekeeping analog multiplexer.

Fast High Voltage Stepping, Accuracy and Settling Time Requirements Tables of stepper voltage commands resident in the IDPU are used for controlling the stepping output voltages through the C\&DH. These tables can be revised and uploaded as necessary throughout the mission. There are several error sources inherent in the stepping process that may affect the values of the stepper voltages and therefore the locations in velocity space actually sampled. These include incomplete dynamic transition and settling, offsets in the C\&DH digital-to-analog converters (DACs), and offsets in the HVPS itself. These offsets can be important at the low end of both the low range and high range of the stepper supplies. These vary from instrument to instrument and over temperature and will also vary in an unknown manner over time due to radiation impact. Therefore the IDPU accommodates separate revisable stepper tables for each DES and DIS. 


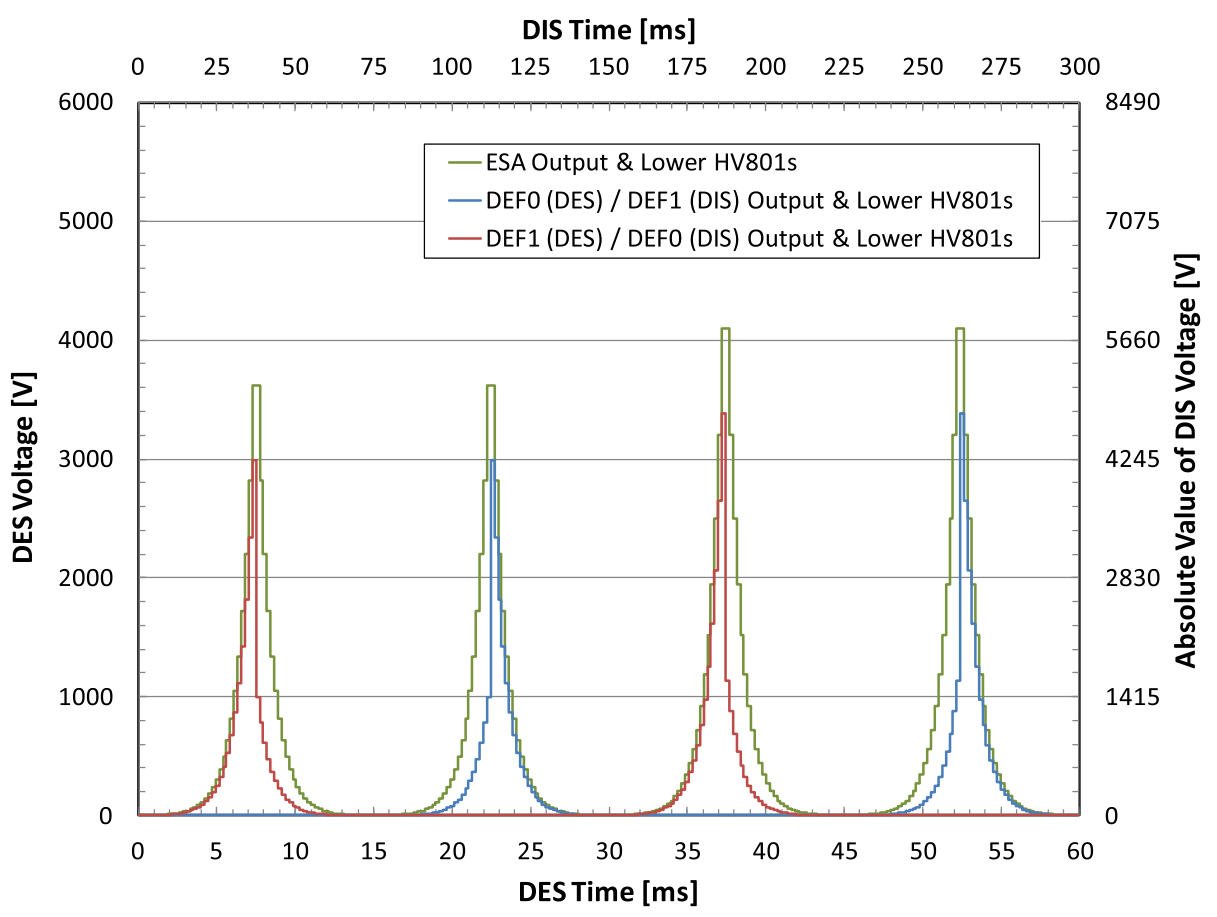

Fig. 27 HVPS stepping output voltages in the DxS HVPS stepping control circuit under flight operation

For DES, the end-to-end offsets (C\&DH and HVPS) were calibrated over temperature for each individual stepping supply output. The calibrations were used to develop distinct stepper tables for every DES at different temperatures in $10^{\circ} \mathrm{C}$ increments. Further, in-flight operational sequences were designed to track these offsets so that the flight stepping tables can be modified as necessary through the life of the mission. Stepper offset errors are thus estimated to be limited to less than $3 \%$. This is the worst case and is realized at the bottom end of the stepper supply's low and high ranges near $10 \mathrm{eV}$ and $300 \mathrm{eV}$, where the offsets will be largest as a fraction of nominal output voltage.

The precision of the end-to-end stepper offset characterization over temperature that was achieved for the DES supplies was not matched in the case of the DIS supplies, with the exception of DIS flight units 11 and 12, which were fully and precisely characterized over temperature. That information together with calibration measurements of the DIS HVPS units and knowledge of the C\&DH digital to analog converters (DACs) based on DES measurements (same part was used) were used to calculate approximate end-to-end calibration factors for DIS. With this method, the offset errors in the DIS stepper voltages are limited to $5 \%$ and mainly localized to the bottom end of the low and high ranges, most significant near $10 \mathrm{eV}$ and $300 \mathrm{eV}$.

Stepper timing is a critical consideration for the DxS high voltage stepping supplies, with transition magnitudes sometimes in excess of 1000 volts. The DxS stepper output voltages during Fast Survey mode are shown in Fig. 27, which shows two complete 128-step low density sweeps (even $E / q$ values followed by odd $E / q$ values). These voltage stepping curves represent the actual target voltage values in the stepping scheme shown in Fig. 26. The average duration of each step in the DES (DIS) application is 234 (1172) $\mu$ s. Each 


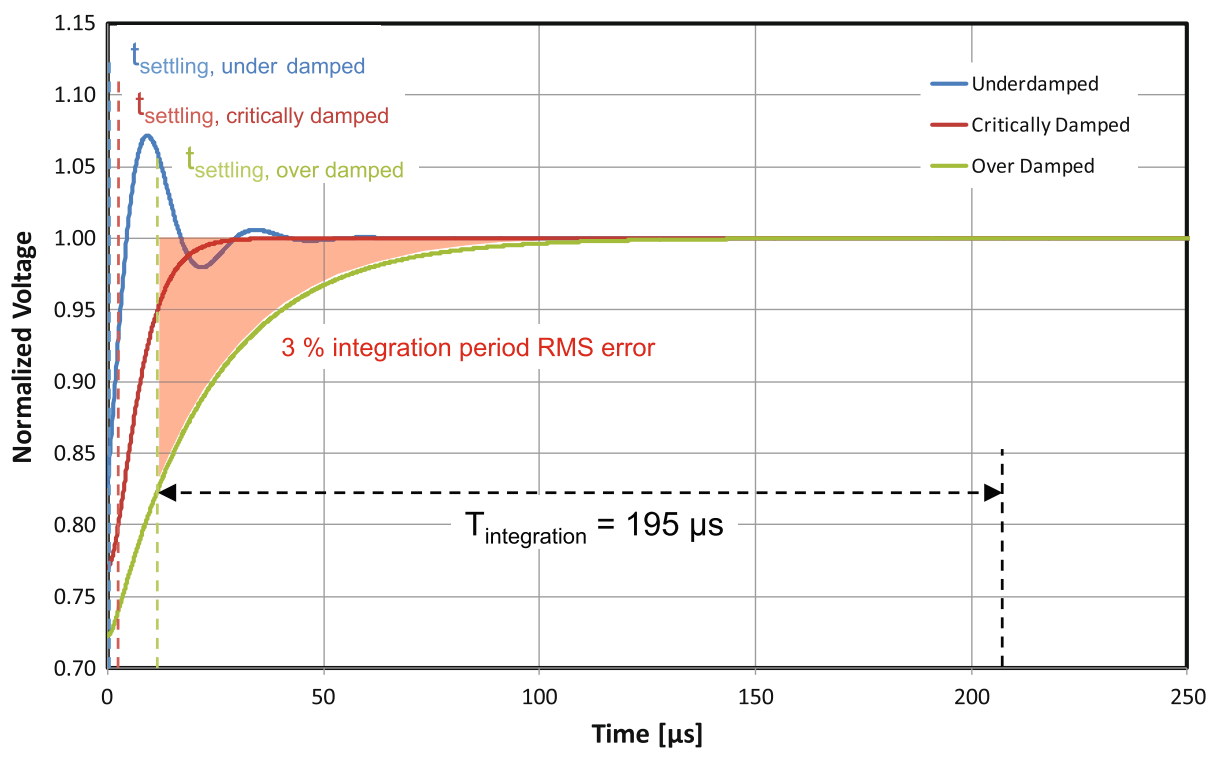

Fig. 28 Dynamic characteristics of model DES stepper voltage transitions

complete low density 128-step DES (DIS) sweep lasts 30 (150) ms. During Fast Survey voltage stepping, it is required for each step that the output values have transitioned and settled sufficiently to ensure acceptably small error in the energy/charge and deflection angle set points during counts integration. For DES (DIS), we have adopted 195 (1000) $\mu$ s per step as the integration interval, leaving on average 39 (172) $\mu \mathrm{s} / \mathrm{step}$, or 4992 (22016) $\mu \mathrm{s} / \mathrm{sweep}$ budgeted for transition and settling. The stepper table enables the available transition and settling budget to be arbitrarily distributed among the 128 steps. This allocation is encoded in the IDPU-resident stepping table.

We have imposed the requirement that the Root Mean Square (RMS) error between target voltage and real voltage resulting from step transitions shall not exceed $3 \%$ of the target voltage throughout the integration interval during which counts are collected. We have performed detailed characterization of the dynamic performance of the DES steppers, including all flight voltage steps and low/high range transitions. Figure 28 shows prototypical cases of a voltage transition that is under damped (blue line), critically damped (red line) and over damped (green line). The over damped case illustrates the limiting acceptable case where the RMS error through the integration interval is $3 \%$. Modeling the real stepper transitions in this way, we have performed fits of the real stepper behavior at each transition, including range change transitions, to one of these instances, thereby parameterizing the steppers' detailed dynamic behavior in each case. We have apportioned the DES settling times accordingly so that our standard DES flight stepper program achieves less than $2 \%$ RMS voltage error on all steps, providing margin against evolution of the dynamic stepper performance under the influence of aging and radiation as the MMS mission progresses.

\subsubsection{Ray Tracing Approach and Tools}

The DES and DIS optics designs were subjected to detailed ray tracing of particle paths, both to guide its design and to compute the anticipated performance and its response to 
impulse sources that are mono-energetic and mono-directional as for laboratory calibration beams, and also for ensembles of particles widely distributed in angle and energy as is the case for the natural plasma populations to be observed in space.

The performance of the DES was calculated using a SIMION based Monte Carlo simulation toolkit that has accurately described the response of several previous space plasma analyzers, including the DES Engineering Test Unit (ETU) (Collinson et al. 2012), the Plasma Electron And Current Experiment (PEACE) on Cluster, and the electron spectrometers of the Analyzer of Space Plasma and EneRgetic Atoms (ASPERA) on the Mars Express and Venus Express missions (Collinson et al. 2009).

The performance of the DIS was calculated using an ISAS electrostatic field modeling program that has accurately predicted the response of several space plasma analyzers, including the DIS ETU, the MAgnetic field and Plasma experiment-Plasma energy Angle and Composition Experiment sensors on the Kaguya lunar mission (Saito et al. 2010; Yokota et al. 2005), and the Mercury Plasma Particle Experiment, a part of the BepiColombo mission (Delcourt et al. 2009).

In both cases, electrostatic fields for each electrode were calculated by solving the Laplace equation with boundary conditions. Models of the DES and DIS optics were built from the mechanical drawings, using cylindrical symmetry to maximize spatial resolution. One million or more particle trajectories were computed for each deflector setting, with initial conditions randomized.

Representative trajectories and energy/angle band pass distributions for DES are shown in Figs. 29, 30 and 31 for DIS. Three deflection states are illustrated, together with the corresponding response of the analyzer in energy-angle space, illustrating that deflection has an asymmetric effect upon the response, as expected given the axial up-down asymmetry of the optics. The significant change in the shape of the energy/angle band pass is characterized by (1) a significant variation in the width of the deflected angle band pass, (2) small or negligible change in the width of $E / q$ band pass and (3) small or negligible change in volume, or total counts in the distribution.

DES Figure 30 shows the incidence pattern of particles passing through the DES optics to the detector input surface in the case of an input beam that is broad in energy and angle. The beam becomes somewhat localized upon a single detector pixel but does not overexpose the pixel. When using a mono-directional and mono-energetic beam, the beam spot becomes smaller in both radius and azimuth on the pixel, leading to premature saturation of the pixel count rate relative to what occurs with the widely distributed excitation. Figure 32 summarizes key aspects of the DES predicted optics response and their variation with deflected angle. The upper panel (Fig. 32a) shows the variation in the predicted width of the DES azimuth band pass $(\Delta \phi, \mathrm{FWHM})$ with deflection angle. $\Delta \phi$ is $6.7^{\circ}$ for the undeflected case, and varies between $4.2^{\circ}$ at $-19.3^{\circ}$ deflection to $8.7^{\circ}$ at $+19^{\circ}$ deflection. This variation in azimuth resolution is a known feature of electrostatic deflection plates (Collinson 2010).

DIS Figure 33 shows the incidence pattern on the MCP detector surface of particles passing through the DIS optics in the case of an input population near $8 \mathrm{keV}$ that is broad in energy/charge and azimuth angle, but narrow in the polar angle. This beam is incident onto a somewhat radially localized asymmetric footprint on the detector (about $4 \mathrm{~mm} \mathrm{FWHM}$ ), in spite of its broadness in energy/charge and deflected angle. This spot size varies with energy in the DIS design, as is discussed further below.

Figure 34 summarizes key aspects of the predicted DIS optics response near $8 \mathrm{keV} / \mathrm{q}$. Not shown in Fig. 33 or 34 is the variation of the peak of the deflected angle bandpass $\left(\phi_{0}\right)$ 

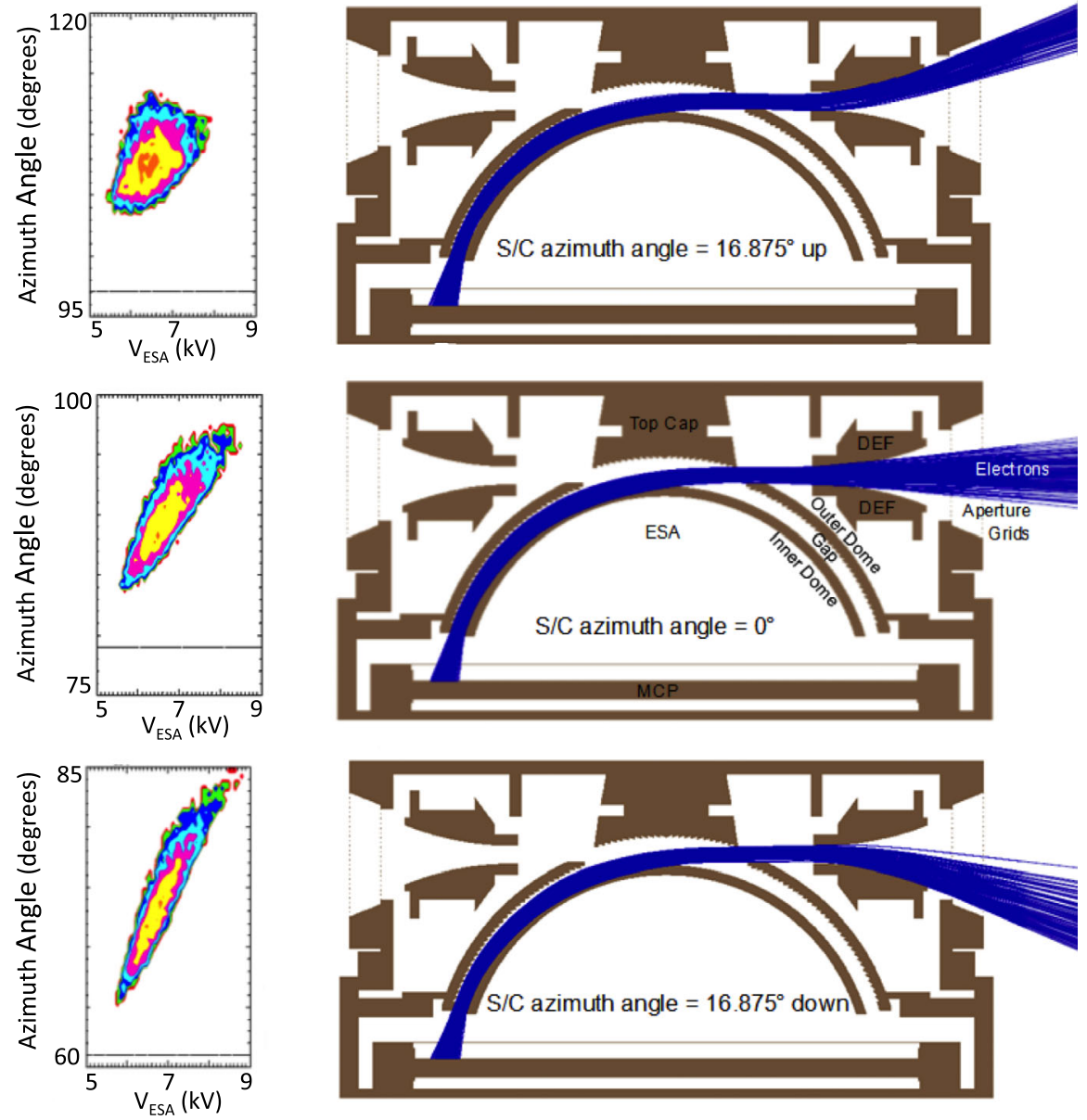

Fig. 29 Example of DES ray-trace simulation results of the energy and azimuth angle response

with voltage applied to the deflector plates. The center of this bandpass is, however, a highly linear and repeatable function of the ratio of applied deflector voltage to ESA voltage. The response of both the upper and lower deflector plates is nearly invariant with energy/charge. Figure 35a shows the variation of the deflected angular resolution $(\Delta \phi$, FWHM) with deflection angle. $\Delta \phi$ is $4.9^{\circ}$ for the undeflected case, and varies between $2.8^{\circ}$ at $-16.9^{\circ}$ deflection to $5.7^{\circ}$ at $+17.1^{\circ}$ deflection.

Figures $32 \mathrm{~b}$ and $34 \mathrm{~b}$ show the DES and DIS deflection angle variation in analyzer constant $\left(k_{\epsilon}\right)$, the constant of proportionality between the peak of the accepted energy/charge bandpass of the analyzer $(E / q)$, and the voltage applied to the ESA, where $E / q=k_{\epsilon} V$. Despite the variations in the shape of the predicted energy-angle response, the simulations predict that the analyzer constant is nearly constant across the range of deflections to be used in flight, with a variation of less then $5 \%$ for DES and less than $1 \%$ for DIS.

Figures 32c and 34c show the DES and DIS variation in the width of the $E / q$ band pass, defined as the FWHM of the $E / q$ band pass divided by the $E / q$ location of the band pass 
Fig. 30 Ray-trace simulations of the DES optics showing the electron spot size on the surface of the MCP generated by the optics under illumination by an isotropic source

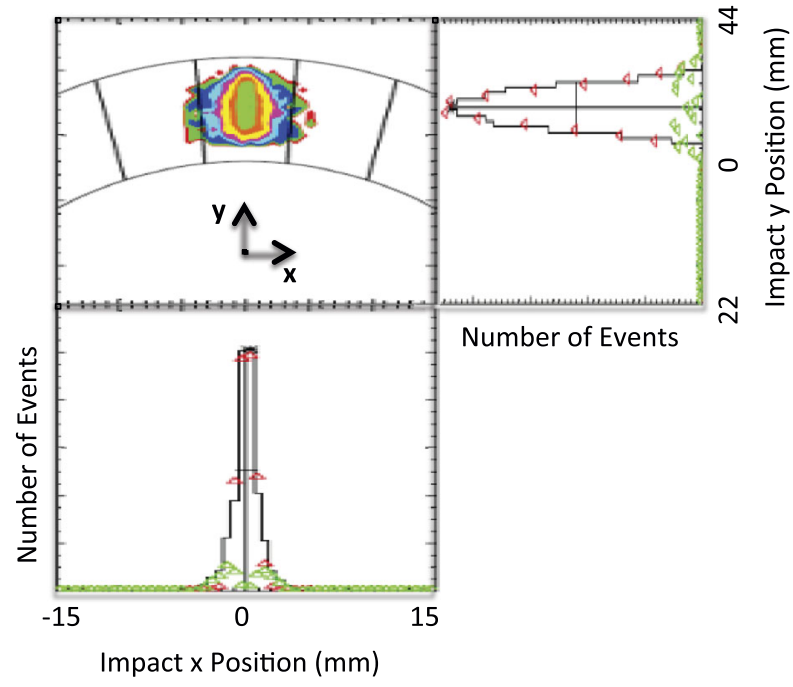

peak. For DES the width of the predicted undeflected energy/charge band pass is $17.4 \%$ and it falls off to $15 \%$ at higher deflection angles. For DIS, the nominal undeflected energy/charge resolution is $11.8 \%$, falling off to $11.6 \%$ at higher deflection angles as the plates impinge slightly on the energy/charge bandpass.

Figures 32d and 34d show the predicted variations in Geometric Factor with deflection angle. The Geometric Factor quantifies DxS sensitivity and is calculated as the product of the geometric aperture area, the width of the polar angle response, the size of the coupled energy and azimuth angle response provided by the optics, the transmission afforded by the grids along the optics path, and the detection efficiency. It is used to perform conversion between counts on the detector to average phase space density within the energy/angle band pass. Computer simulations can only determine the raw physical geometric factor resulting from the optics. Other effects such as detection efficiencies and grid transparency must be estimated. The solid line in the figures is the raw output of the ray tracing simulation and shows this physical geometric factor to be near $1 \times 10^{-3} \mathrm{~cm}^{2}$ for DIS. Grid transparency and detector efficiency have been estimated for two energies $(20 \mathrm{eV}$, dashed line and $30 \mathrm{keV}$, dotted line) to plot a prediction of the actual Geometric Factor. Full details using laboratory data from the ETU can be found in Collinson et al. (2012).

An additional ray tracing study was performed at GSFC using the SIMION program in order to study the impact of leakage of the electric field produced by the unshielded top of the MCP detector assembly (held near $-2 \mathrm{kV}$ ) into the lower portion of the ESA. Though strongly attenuated, the attractive potential of the MCP assembly penetrates a short distance into the ESA with the result that some particles pass through that otherwise would not. Like the first ray tracing study, the subsequent SIMION study illuminated the DIS model aperture with ions that have broad spatial distribution (fill the aperture) and ranges of energies/charge and azimuth angles that are large compared with the instrument band passes but a range of polar angles that is narrow. The ray tracing predicts impacts on instrument performance as shown in Fig. 35, where various performance parameters are plotted versus $E / q$. None of these parameters would be expected to vary with $E / q$ in the case where the MCP input grid was grounded. The values seen at high $E / q$ (above $1 \mathrm{kV}$ ) are not significantly influenced by the leakage of MCP field into the ESA and therefore represent behavior to be expected in the case where the MCP input grid is grounded. In Fig. 35a the predicted geometric factor 

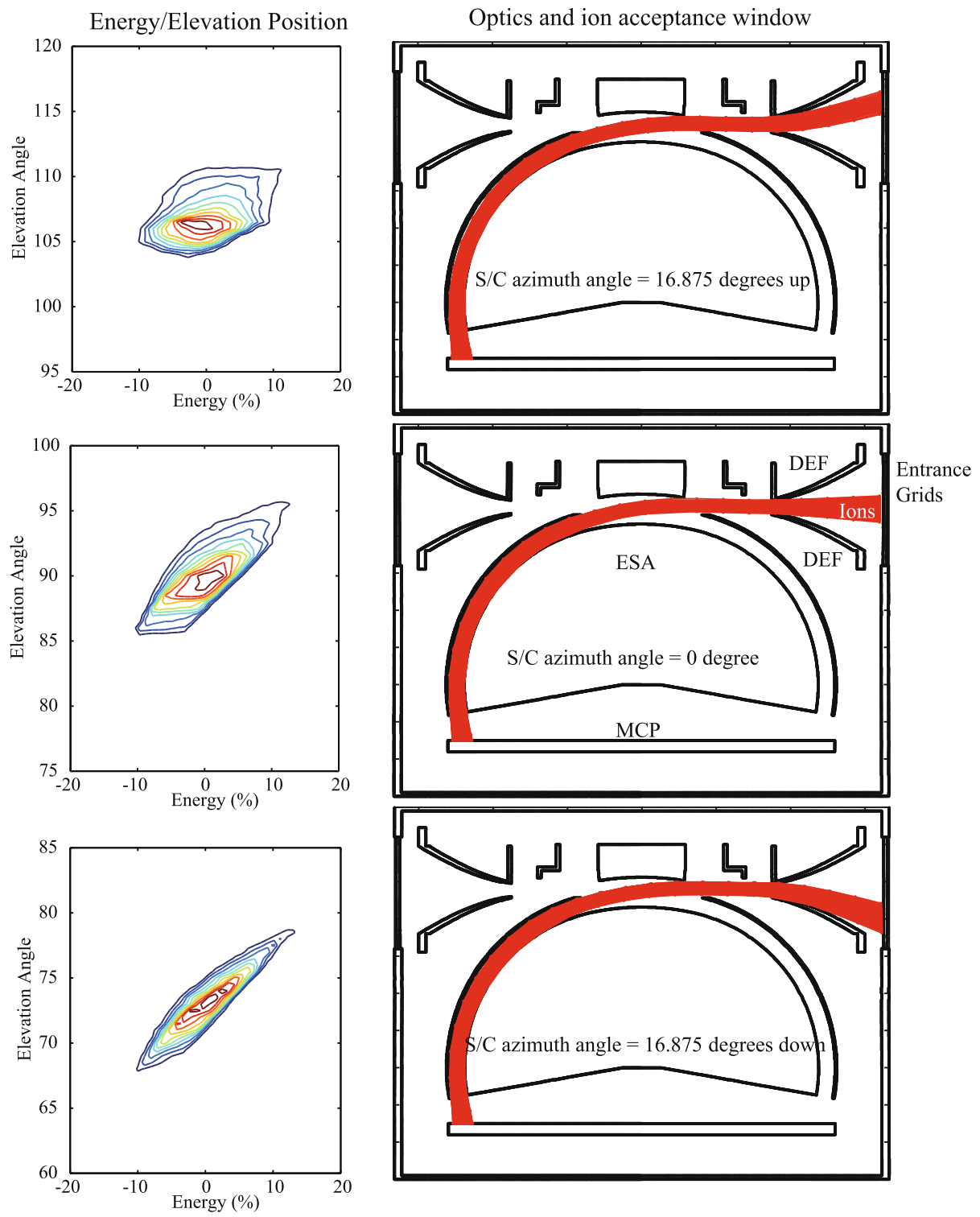

Fig. 31 Ray-tracing simulation trajectories used to predict the performance of FPI-DIS

assuming unity efficiency and no decrement from grids in the flight path is shown to increase from near $1 \times 10^{-3} \mathrm{~cm}^{2} \mathrm{sreV} / \mathrm{eV}$ for $E / q$ above $1 \mathrm{kV}$ to near $1.6 \times 10^{-3} \mathrm{~cm}^{2} \mathrm{sreV} / \mathrm{eV}$ for $E / q$ near $1 \mathrm{eV} / \mathrm{q}$, a $60 \%$ increase from nominal. The value at higher energies is consistent with the prediction of the original ISAS ray tracing study (Fig. 35d, solid line). Figure 35b shows predicted variation of the analyzer constant $(E / q V)$ and energy band pass $(d E / E)$. While the analyzer constant shows only a few percent variation across $E / q$, the energy band pass is seen to increase from its nominal value of near $12 \%$ for $E / q$ above $1 \mathrm{kV}$ up to $17 \%$ at $E / q$ of $1 \mathrm{eV}$, an increase of $42 \%$. Figure $35 \mathrm{c}$ shows the center and width of the deflected 
Fig. 32 Predicted response of the FPI-DES sensor from Monte Carlo ray tracing optics simulation

Fig. 33 Spatial distributions of particle arrivals on DIS MCPs, as predicted by numerical electrostatic ray tracing
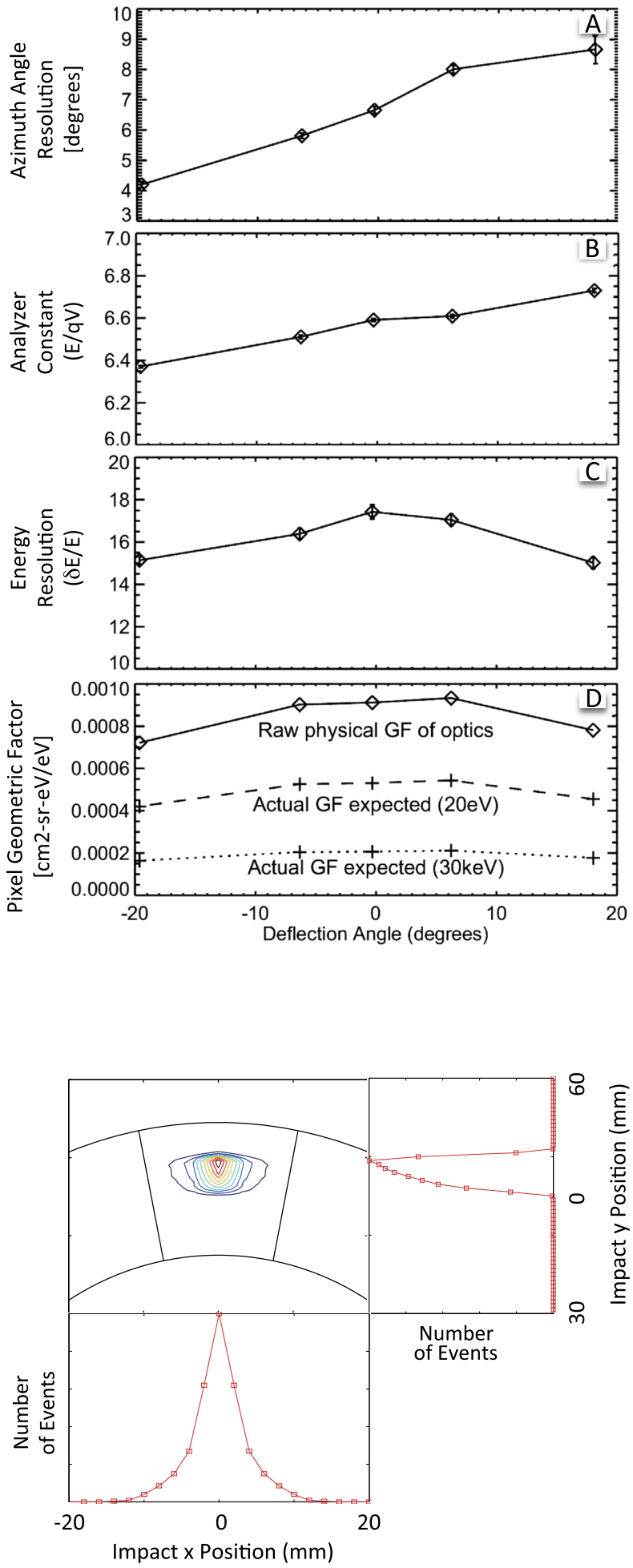
Fig. 34 Predicted DIS response parameters near $8 \mathrm{keV} / \mathrm{q}$, based on numerical ray tracing as functions of the electrostatic deflection angle. Panel A shows the FWHM of the azimuthal angle response; Panel $\mathbf{B}$ shows the analyzer constant $((E / q$ VESA) peak); Panel $\mathbf{C}$ shows the FWHM (\%) of the energy/charge response; Panel $\mathbf{D}$ shows the geometric factor. In Panel D, the solid line assumes both unity detection efficiency and transmission through grids; the dashed and dotted lines include $90 \%$ transmissions for all grids and estimated detection efficiencies at $20 \mathrm{eV}$ and $30 \mathrm{keV}$, respectively
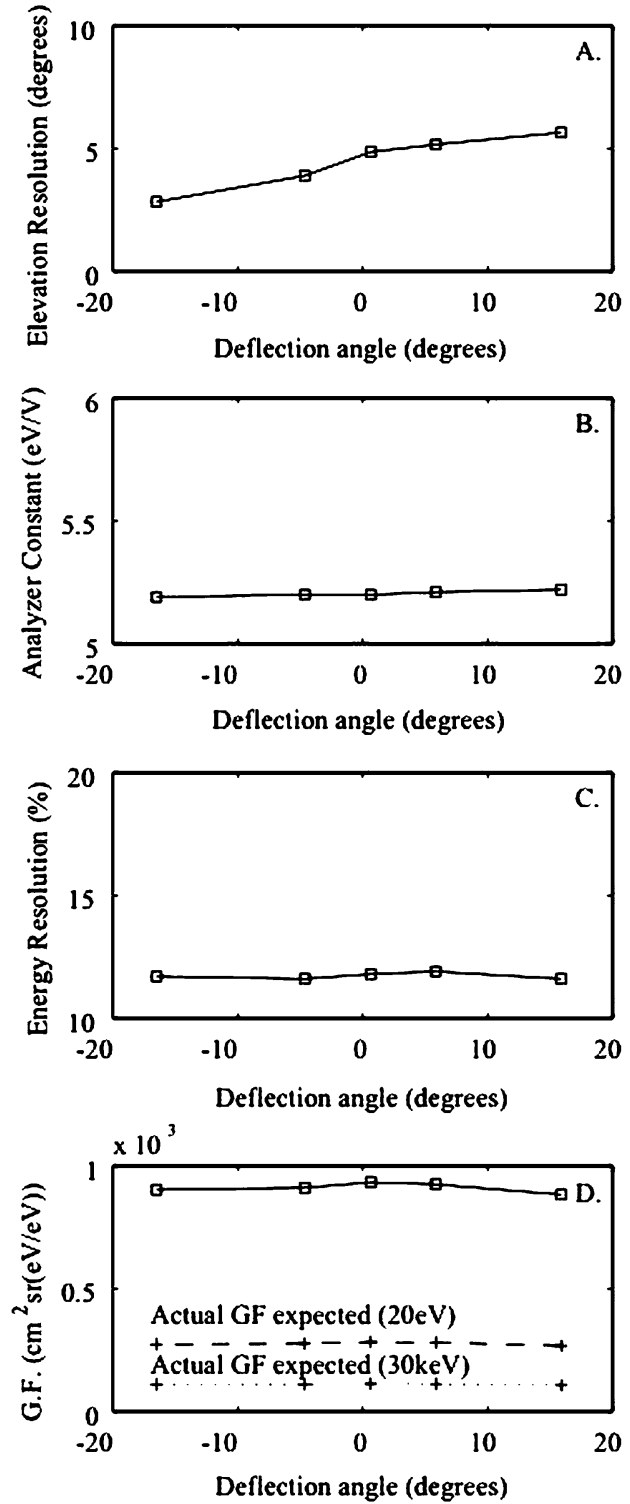

angle band pass. The center shifts about $0.5^{\circ}$ toward more positive angles (viewing away from MCP plane) at the lowest values of $E / q$ and the width increases by about $0.6^{\circ}$ (about $10 \%$ ) from high to low $E / q$. Together, the $42 \%$ increase in the energy band pass and the $10 \%$ increase in the deflected angle band pass nearly, but not quite fully, account for the $60 \%$ increase in predicted geometric factor from high to low energy.

There is another important effect of the lack of shielding between the MCP high voltage and the ESA. Figure 36 shows representative rays from the SIMION study illustrating the electrostatic focusing properties near the exit of the ESA. The variation in radial spot size is large and minimizes within the DIS $E / q$ range near $500 \mathrm{~V}$ (Fig. 36c). This effect will have the important and unfortunate impact that the saturation performance of the MCP will 
Fig. 35 Predicted DIS response parameters as functions of ion energy per charge, based on numerical ray tracing. Panel A shows the geometric factor; Panel B shows both the analyzer constant ( $E / q$ VESA) peak; blue solid circles) plotted on the left axis and the fractional width of the $E / q$ band pass $(d E / E$; red open circles) plotted on the right axis; Panel $\mathbf{C}$ shows the location (black open circles; left axis) and width (red filled circles; right axis) of the polar angle response peak for the case of no voltage applied to the deflectors

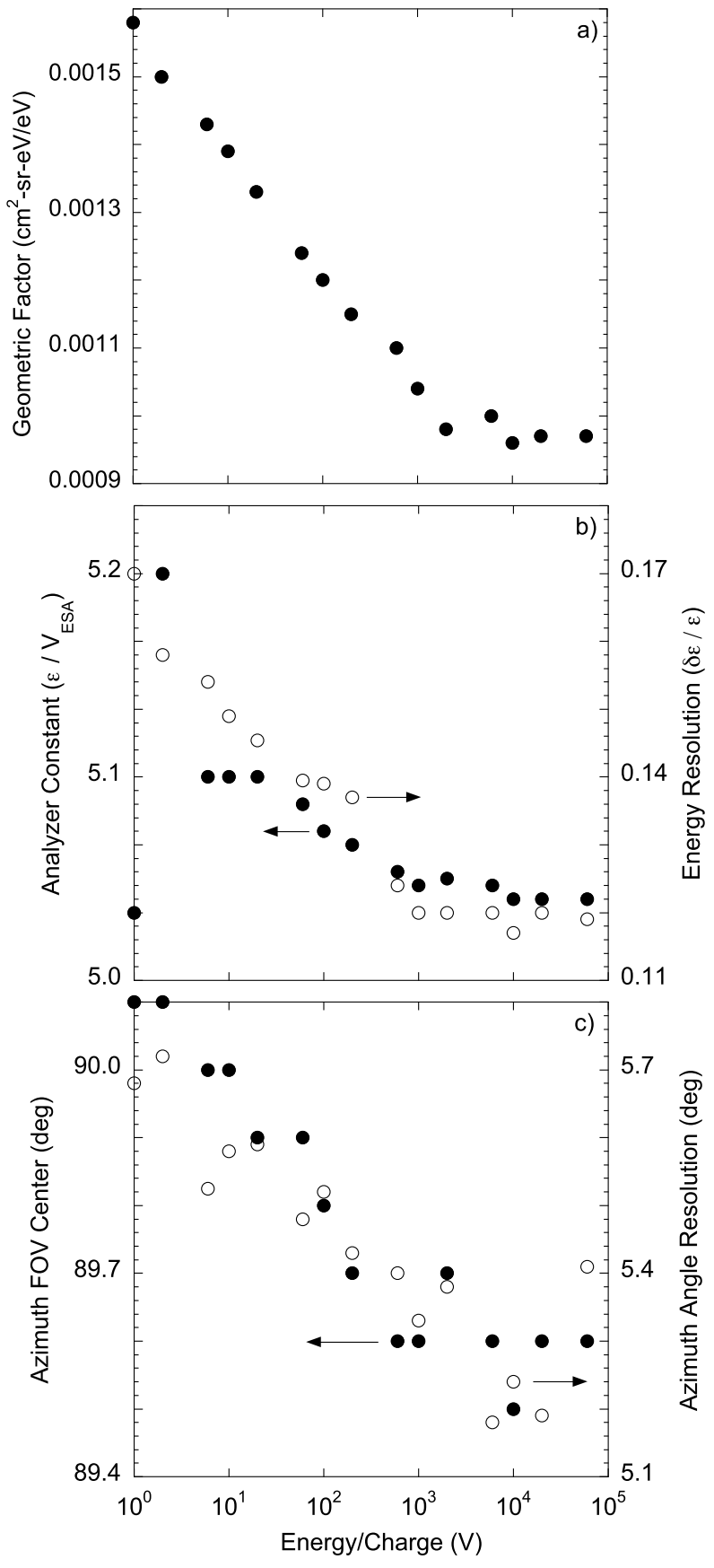

be a function of $E / q$, becoming most severe for DIS near $500 \mathrm{eV} / \mathrm{q}$. This is because MCP detectors saturate on the basis of event rate per unit area. Since the beam spot is smallest near $500 \mathrm{eV}$ this will be the energy/charge at which the onset of event rate saturation will occur 

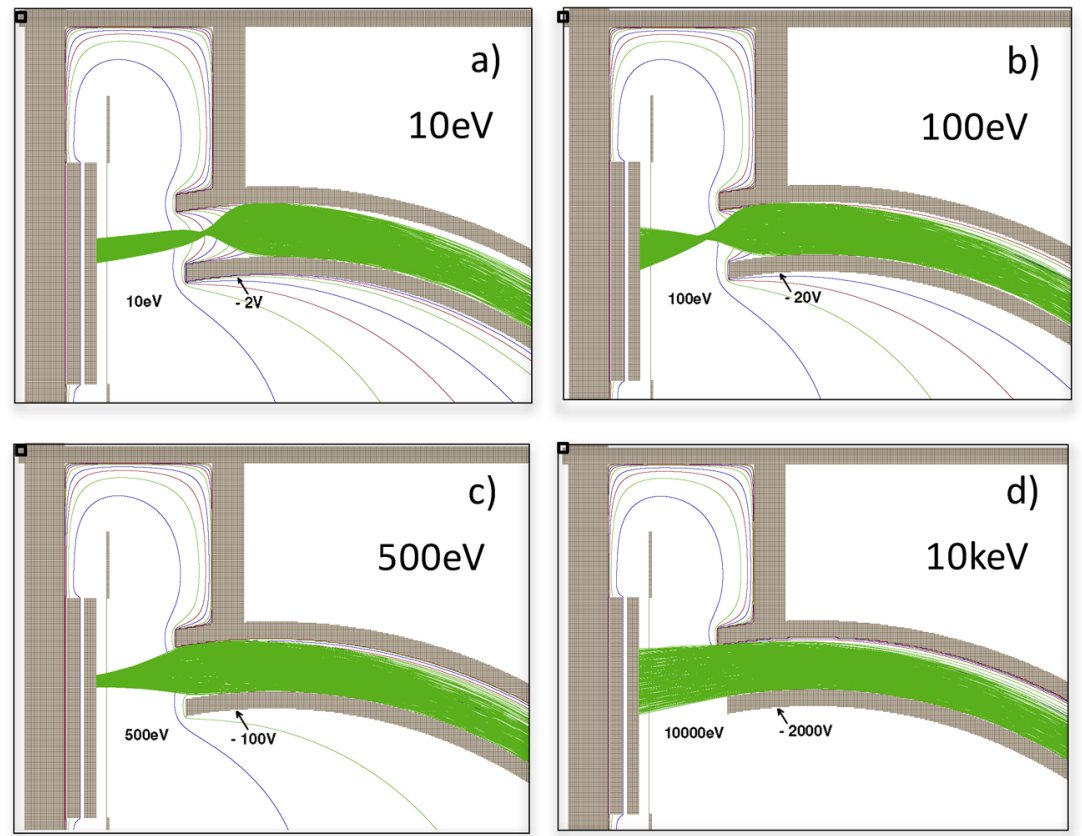

Fig. 36 Electrostatic focusing properties for transmitted ions are displayed in the vicinity of the DIS ESA exit aperture for several values of $E / q$

at the lowest event rates. If unaccounted for, this effect will produce incorrect distribution function dependence on energy at large event rates.

In summary, these optics simulations showed that the response of the DES and DIS spectrometers were predicted to meet or exceed the required performance parameters.

\subsection{Instrument Data Processing Unit}

\subsubsection{IDPU Overview and Implementation}

The FPI IDPU is a block redundant system that commands the four DES and DIS units on an MMS Observatory. It consists of a Data Processing Card (DPC), a Low Voltage Power Converter (LVPC) board and a Spectrometer Power Switch Card (SPSC). The IDPU performs numerous functions, including: (i) command and telemetry processing, (ii) power switching for the spectrometers, (iii), commanding the high voltage stepping supplies responsible for energy and angle selection, (iv) collection and organization of spectrometer counts data into skymaps, (v) calculation of metrics (trigger terms and trigger numbers) indicating the statistical quality of the sky map data, and (vi) acquisition of data in engineering mode as part of macro execution during MCP ramps and other macro driven calibration activities. These functions are completed in real time so the results can be stored in the CIDP Mass Memory Module (MMM) synchronously with their generation. Table 3 provides a summary of the modes and functionality of the FPI IDPU.

The IDPU receives data and commands from the ground via the CIDP. The IDPU directly controls and collects data from and the four DES and DIS units. In response to the promptings of the IDPU, each dual spectrometer executes ESA and deflector voltage stepping, collects data and provides the data in the form of counts per integration interval. The 
Table 3 IDPU modes and functions

\begin{tabular}{ll}
\hline Name & Description \\
\hline Auto gain control & $\begin{array}{l}\text { When enabled, steady unmodulated high count rates may trigger reduced } \\
\text { gain with recovery to full gain when lower rates are restored. This is }\end{array}$ \\
& $\begin{array}{l}\text { essentially radiation belt protection but also serves to detect discharges, } \\
\text { should they occur }\end{array}$ \\
Optimized sampling (pruning) & $\begin{array}{l}\text { Remove/combine samples to enforce uniform solid angle coverage. This is } \\
\text { typically used only in calculation of Trigger terms and numbers }\end{array}$ \\
Slow survey mode & $\begin{array}{l}\text { Single spectrometer box for DES and DIS used to build skymap over } 3 \\
\text { spins }\end{array}$ \\
Fast survey/burst mode & $\begin{array}{l}\text { Nominal FPI mode. All FPI spectrometers operating in concert at full } \\
\text { speed. Both burst data products and fast survey data products are produced } \\
\text { in this mode }\end{array}$ \\
A set of pseudo-moments (sums over energy and angle) defined in Table 6 \\
and reported in Telemetry with skymaps as high time resolution (30 ms \\
electrons; 150 ms ions) elements of the FS data \\
Statistical quantities computed based on the trigger terms and reported on \\
10 s intervals \\
Utilizes application specific integrated circuit to apply wavelet \\
compression as commanded, targeting fixed data volume per telemetry \\
frame \\
Enables execution of individual instrument commands from any source, \\
including ground. Used for several routine activities, real time \\
troubleshooting and table uploads
\end{tabular}

IDPU assembles these counts into both Burst and Fast Survey skymaps and produces data streams to the CIDP of the following types:

Burst and Fast Survey Skymaps The burst skymaps comprise the raw counts from DES and DIS acquired in Fast Survey mode. These are count rate arrays (32 energy $\times 32$ azimuth angle $\times 16$ pixels) accumulated every $30 \mathrm{~ms}$ for DES and $150 \mathrm{~ms}$ for DIS. To form the Fast Survey skymaps, the IDPU sums 150 (DES) or 30 (DIS) Burst Skymaps yielding a $4.5 \mathrm{~s}$ product in both cases. The Fast Survey sky map data are de-spun and then binned onboard into a De-spun Body Spacecraft Coordinate System (DBCS) that is very close to Geocentric Solar Ecliptic (GSE) coordinates (within 2-3 degrees). The Fast Survey sky map have pixels that feature contributions from more than one sensor or deflection state, owing to the Fast Survey $4.5 \mathrm{~s}$ integration time.

Burst Quality Indices (BQI) FPI trigger terms are defined as seven weighted sums (three for electrons and four for ions) that are performed in the IDPU over the DES and DIS Burst skymaps at the burst time resolution (30 ms for DES and $150 \mathrm{~ms}$ for DIS). These sums are intended to represent quantities analogous to physical moments of the electron and ion phase space density distributions. A trigger data packet is delivered by the IDPU in response to a periodic message from the CIDP that is received nominally every $10 \mathrm{~s}$. This packet contains the time series of trigger terms for electrons and ions, in addition to a set of eleven trigger numbers for each species that represents the means and variances of the trigger term time series.

Slow Survey Skymaps When operating in Slow Survey mode, sky maps are constructed using a single dual spectrometer unit for each species operating in a conventional spin-scan mode without deflection. The operating DxS changes from orbit to orbit. Three spins are used to accumulate a single Slow Survey sky map. 
Calibration Data For each orbit, a sequence of calibration measurements is made in an interval of 10 minutes at the boundary between the end of the science ROI and the start of the inbound Slow Survey portion of the orbit. The result is a set of data characterizing the health and relative sensitivity of the MCP detectors.

Housekeeping Data Engineering parameters, referred to collectively as Housekeeping data, are routinely monitored and reported in telemetry from the IDPU and through the IDPU from the spectrometers. These parameters include voltages and currents characterizing the engineering performance of the system. Trending and reviewing housekeeping data will be a critical element of system management and maintenance.

\subsubsection{Functions and Tables of the IDPU Data Processing Card}

The IDPU is limited to functions hard coded in an FPGA state machine. Many instrument parameters are stored in up-loadable tables and/or in command registers that can be adjusted based on experience and the results of on-orbit calibration sequences. In addition the wavelet compression is performed via a GSFC-developed application specific integrated circuit (ASIC) with low power consumption.

High Voltage Stepper Tables and K-Tables While the details vary with context, all deflection and energy selection is controlled using high voltage stepping tables. These tables supply timing information and index values used to inform the IDPU's building of sky maps from returned instrument counts. Other tables supply MCP biases used in automated flight calibration sequences. In addition, FPI tracks two sets of values for each species' sequence of energy targets. These differ in their energy scaling. For every energy $E$, there is a densitytype quantity that results from scaling the counts with $1 / \sqrt{E}$ and a pressure-type quantity that results from scaling the counts with $\sqrt{E}$. As with high voltage steppers and compression configurations these scaling quantities are encoded in configurable operating tables.

Fast Survey Stepper Tables Four tables must be configured for each spacecraft to operate in Fast Survey. Each species continuously alternates between two 128-row tables. Out of a full sequence of 64 energy-selection filters, assigned indices $0-63$, energies $0,2, \ldots, 62$ form the even and $1,3, \ldots, 63$ form the odd set. The 128 steps of each single-parity table are comprised of: a sweep through 32 energies at deflection state 0 , then corresponding sweeps at each of states 1-3. Each dual spectrometer is commanded with independently configured words at each step, allowing independent adjustments to be made over lifetime, temperature, etc. All dual spectrometers of a given species on a given spacecraft must use common timing and indexing values at each step.

Slow Survey Stepper Tables Slow Survey stepper timing is spin-synchronous, only the dual spectrometers in a single instrument deck quadrant are used on any orbit in slow survey mode and no deflection is used. The pattern of stepping then only involves sweeping the even and odd energies. This two-sweep pattern is repeated for each of 16 equal azimuthal sectors, fixed with respect to the Sun direction. Counts from common azimuth sectors are summed. Once again, four tables must be configured for each spacecraft to operate in Slow Survey: two species by two parities, 32 rows each. The active-quadrant dual spectrometer of a species, per spacecraft, is sent the same command word, regardless of active quadrant. A simple energy-bin index instructs the building of summed sky maps. 
Ensemble Calibration Mode Stepper Tables The Ensemble Calibration mode consists of 16 spins of observation, each potentially at a different MCP voltage. During Ensemble Calibration, each DxS sensor executes a 32-step $E / q$ sweep 16 times during each spin. The two 32-row stepper tables (one for ions and one for electrons) needed per spacecraft to encode the $E / q$ targets are re-configurable. Operating at constant MCP voltage, the Ensemble Calibration data is used to measure, with maximal counting statistics possible, the overlap region between the high and low range of the stepper supplies in order to identify and correct for evolving offsets.

Trigger Calculation K-Tables In calculating FPI trigger term series from Burst skymaps, a total of eight 32-element tables are required per spacecraft: 2 types $\times 2$ species $\times 2$ parities. The types are density-like, scaling as $1 / \sqrt{(E / q)}$ and pressure-like, scaling as $\sqrt{(E / q)}$. Since these are dependent only on energy targets, they are likely to remain constant across all spacecraft and over time. The species are electrons (DES) and ions (DIS) and the parities are associated with the even and odd segments of the $E / q$ sweep programs.

Unit Vectors and Pruning Three unit-vector tables used in on-board trigger calculations encode the spacecraft frame $\mathrm{X}, \mathrm{Y}$, and $\mathrm{Z}$ components of the unit vectors associated with the look direction array of 32 spacecraft azimuth angles $\times 16$ polar angles. These represent the nominal look-directions of every FPI pixel when operating in Fast Survey mode. It is likely that these tables will not be re-configured, and are the same for all spacecraft.

Two additional $32 \times 16$ spacecraft frame maps, referred to as pruning maps, are required to configure the trigger calculations on each spacecraft. For each species, a separate binary map encodes which of its 512 pixels will be included in the metrics. This feature is useful in mitigating the effects of oversampling near the spacecraft spin axes and to remove troublesome pixels, for the trigger term calculations.

Compression and Trigger-Encoding Look-Up Tables (LUTs) Eight compression configuration tables are required per spacecraft to configure the compression of sky maps from 2 species $\times 3$ types +2 diagnostic cases. These configurations are applied to the Consultative Committee for Space Data Systems (CCSDS) compression Application Specific Integrated Circuits (ASICs) as appropriate. Similarly, three additional LUTs are used per spacecraft to encode trigger data samples to 8 bits. Only the three types of sky map data are CCSDS compressed: Burst, Fast Survey, and Slow Survey. Each spacecraft requires a table for each type and species and can accommodate two more diagnostic tables. FPI 8-bit-encodes all Trigger Terms in Fast Survey data using the LUT for relating trigger terms (from both species) to 0-255 values. All mean-type trigger "numbers" are encoded using the second LUT, and all variance-type numbers use the third LUT.

IDPU Low Voltage Power Converter The two redundant IDPU LVPCs receive $+32 \mathrm{~V}$ bus power from the MMS CIDP. Each one provides two regulated low voltage outputs referenced to the primary side ground and five isolated, tightly regulated, low voltage outputs referenced to secondary side grounds. Of these seven individual output voltages, four low power outputs which include the $+10 \mathrm{~V},+5 \mathrm{~V},+8 \mathrm{~V}$, and $-5 \mathrm{~V}$ levels are used by the Spectrometer Power Switch Card (SPSC) and are derived from a common multiple secondary, pulse width modulated, voltage mode controlled bias network that provides the required ground isolation and pre-regulation functions. The $+10 \mathrm{~V}$ and $+5 \mathrm{~V}$ outputs are regulated to $\pm 5 \%$. The $+8 \mathrm{~V}$ and $-5 \mathrm{~V}$ outputs utilize subsequent discrete linear regulator networks that provide $\pm 1 \%$ line/load regulation and over-current protection. The higher power outputs which include the $+3.33 \mathrm{~V},+2.5 \mathrm{~V}$, and $1.9 \mathrm{~V}$ levels are used by the IDPU DPC. 
These voltages are derived from three independent pulse width modulated, current-mode controlled forward power stages. The $2.5 \mathrm{~V}$ and $1.9 \mathrm{~V}$ outputs also provide remote sensing capability to ensure point-of-load regulation. The LVPC provides differential mode and common mode filtering on both primary and secondary sides of the $200 \mathrm{KHz}$ switching converter stages. Additional features within the LVPC include input bus inrush current limiting, input bus under/over voltage protection, and output voltage sequencing during both turn on and turn off.

IDPU Spectrometer Power Switch Card The IDPU SPSC receives $+32 \mathrm{~V}$ bus power from the MMS CIDP and contains eight independent solid-state switching circuits for ON/OFF commandable distribution of the $+32 \mathrm{~V}$ bus voltage to each of the eight spectrometers. The status of these switches is controlled by commands from the IDPU DPC. Each spectrometer power switch utilizes a dual Field Effect Transistor arrangement such that both the forward and return current paths may be opened or closed as required. This arrangement enables the complex cross strapping of spectrometer power to be achieved by the two independent on-board SPSCs while maintaining the required control of all power return currents. Each switch network includes a soft turn on characteristic for limiting peak inrush currents to each spectrometer. Each switch network also includes an over-current trip off network for protection in the event of overload conditions. The SPSC provides telemetry monitoring of the associated voltages and currents with each of the $+32 \mathrm{~V}$ spectrometer power switch networks and monitors the bus voltage to the IDPU and the current draw by the IDPU subcomponents. Ground isolation is maintained between the primary referenced switch networks and the secondary referenced monitoring electronics by utilization of a command and telemetry exchange through magnetically coupled signal paths. This technique was derived specifically for this MMS application and provides capability for complete frame data (all channels) or dwell functions (single channel) monitoring as selected by the Processor Card interface.

\section{FPI Instrument Calibration}

The objectives of calibration are to obtain and retain accurate answers to the questions: (1) Where in velocity space $(E / q, 2 \mathrm{D}$ angle) are we sampling and with what sensitivity and (2) What sources of spurious counts (crosstalk, noise) are present and can they be mitigated?

Velocity space sample locations are determined by the spectrometer electro-optical properties and the voltages applied by the stepping high voltage supplies in addition to the spectrometer orientation on the spacecraft. The goal in flight is to maintain consistency in that sampling as closely as possible. The sensitivity is determined by the electro-optical properties and the detection efficiency of each MCP/anode/amplifier combination, including the interaction between MCP pulse height distribution and amplifier discrimination threshold.

Pixel noise sources, aside from classical Poisson counting noise, include scattering crosstalk from particles at or above the MCP input surface, anode-to-anode capacitive cross talk, noise injected at the preamplifiers by the MCP supply or the stepper supplies, crosstalk at preamplifier inputs and other elements of system EMI. Spurious counts are associated with spontaneous electron emission (dark counts), noisy pixels, and external sources such as photoelectrons and penetrating radiation. These noise sources are observable and-in many cases - are quantifiable in laboratory calibration and sometimes in flight.

FPI conducted a comprehensive baseline ground calibration program to characterize these quantities and designed a robust inflight calibration procedure. Performed on every flight unit, the ground calibration provided characterizations of the performance of the DxS 
optical components, high voltage stepper supplies and detector system performance. Optics and detection system characteristics were documented at two levels named the Gold and Standard levels. Gold level calibration was more extensive and performed for one DIS and one DES of each Observatory suite. Standard calibration was performed on the remaining six DxSs of each suite to confirm nominal response over the full range of angles and energies.

\subsection{Approach to Sensor Calibration}

During flight operations, data from eight electrostatic analyzers are combined into a single 3D phase space distribution. To ensure the data from each set of eight spectrometers can be corrected to yield an accurate output for a given input, an extensive laboratory and inflight calibration regimen was developed to appropriately balance operating parameters and to identify and correct for instrument variability and noise. While all DES are nominally identical, as are all DIS, individual performance varies due to the variations in parts, detectors, surface machining/finishing, and assembly. Variations in parts-MCP detectors in particular — and noise sources can change through mission life.

\subsubsection{Threshold Sweeps}

Figure 37 is a schematic view of the DxS signal environment at the inputs and outputs of the A121 preamplifiers/discriminators. Figure 37a illustrates the expected contributions to the pulse height distributions. The gain is defined to be $50 \%$ of the signal count rate. This definition makes the assumption that the peak of the distribution is at the $50 \%$ line (i.e., a symmetric distribution), which is not strictly applicable but sufficiently close for the purposes of estimating gain. The red Gaussian-like distribution represents the prime signal and is slightly lower in gain than the two (orange, yellow) scattering cross talk contributors (from the ESA and MCP surfaces) directly to its right, owing to a presumed smaller rate from scattered than from primary events and thereby possibly higher gain. The green pulse height distribution is owing to capacitive anode-to-anode cross talk and is a direct fractional image of the primary signal. Other sources of pulses include dark counts (spontaneous emissions of MCP pulses), noise picked up from the HVPS, A121 output-to-input cross talk (re-triggering), MCP-to-anode cross talk and partial pulse collection/cloud spreading cross talk. Ideally, all MCP prime signal pulses fall above the discriminator threshold and are detected only by the anode associated with the MCP. This requires the pulse height distribution to have both a sufficiently large mean gain compared to the value of the detection discriminator threshold and to be sufficiently narrow. A finite signal loss results when portions of the MCP pulse height distribution falls below threshold. Further, the cross-talk terms must be sufficiently low compared to the detection discriminator threshold.

Figure $37 \mathrm{~b}$ shows the output pulse rates expected for each of the pulse height distributions as a function of the discrimination threshold. The threshold is expressed in the same units as the pulse heights that comprise the abscissa of Fig. 37a. Conceptually, the count rates are integrals of the pulse height distributions with a domain of integration from the set threshold value to infinity.

Figure 37c shows the schematic sum of all signals at the primary channel in red and all crosstalk and noise signals at neighboring channels in gray. This is what is measured during calibration and enables the isolation and direct measurement of the important detector system quantities: MCP gain, magnitude of anode-to-anode capacitive cross talk, cross talk from scattering above the MCP, the margin against output-to-input cross talk, and high voltage and other sources of noise. This "threshold sweep", where we plot the rates of the 
Fig. 37 Salient characteristics of thresholded multi-channel electron multiplier based detection system are illustrated

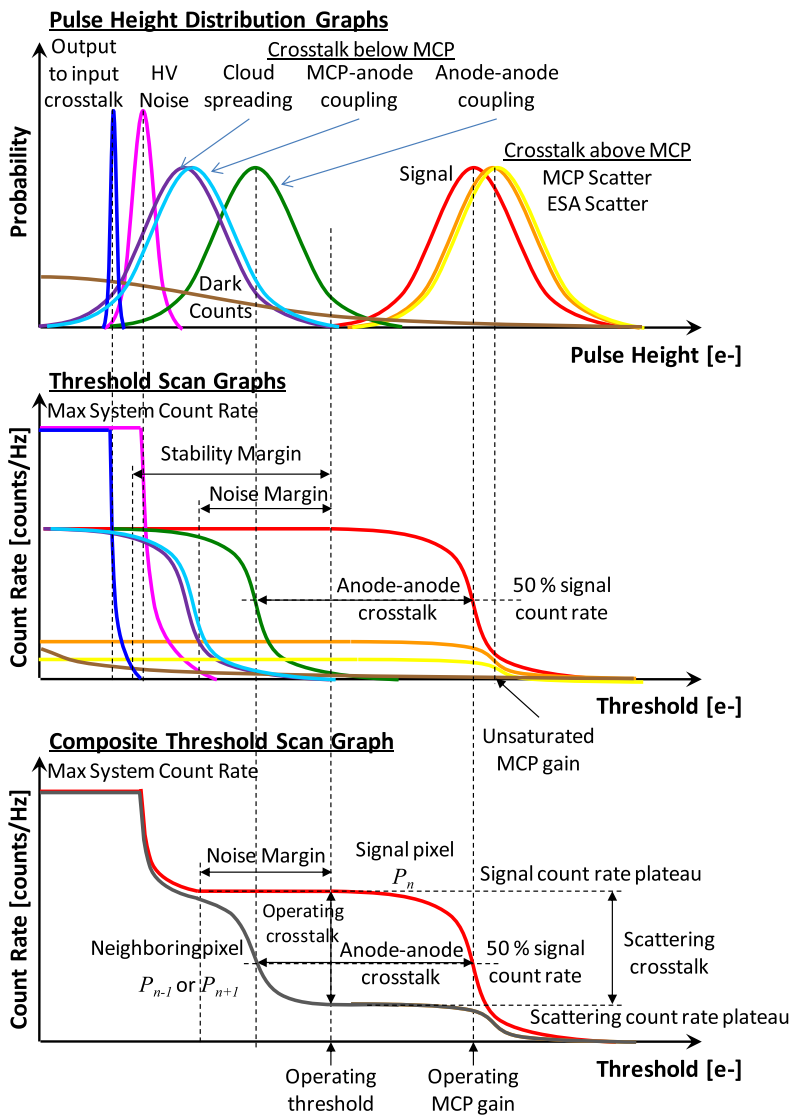

sixteen anodes in a DxS sensor versus applied threshold, are performed in the laboratory and in flight, both with charged particle stimulus and under dark conditions. In flight, unlike in the laboratory, many channels are stimulated simultaneously, rendering the interpretation more complicated but still tractable.

Sensor performance then is strongly dependent upon the detection system operating point, which is defined by the applied MCP high voltage-determining the MCP pulse height distribution (gain) - and the pre-amplifier threshold setting. FPI exercises great care to define, track, and maintain DxS operating points during ground calibration and in flight. Using threshold sweeps, special operating point calibrations measure the response of all sixteen pixels in a sensor head as functions of preamplifier discriminator threshold and MCP voltage in the presence of a signal source. On the ground, the signal source was a monoenergetic, collimated beam, directed into a single pixel, while in flight the signal source will be the external plasma, typically directed at many pixels. On the ground, threshold scans were performed at several (typically seven) MCP voltages while in flight threshold scans are performed at two voltages.

\subsubsection{Calibration Procedure Overview}

The full calibration program included determination of the operating point (MCP gain and voltage, and discriminator threshold), characterization of signal loss due to MCP 
gain variation, relative pixel to pixel MCP efficiency variation, cross talk, noise, coupled energy/azimuth-angle band pass locations, shapes and amplitudes and their dependence on high voltage stepper supply states, and the locations and shapes of the polar angle band passes and any dependence they have on high voltage stepper supply state. The ground calibration also characterized system dead time and the effectiveness of UV rejection.

The general ground-based calibration procedure was (1) illuminate sensor with a beam sufficiently broad to fill the entrance aperture and sufficiently narrow in both energy and 2D angle to approximate an impulse stimuli; (2) execute $V_{E S A}$ and $V_{D E F}$ sweeps centered at voltages to pass the beam particles and wide enough to capture the $V_{E S A} / V_{D E F}$ band pass; and (3) mechanically articulate the sensor to measure beam incidence over the complete angular range of pixel response. The zero deflection state-used in Slow Survey mode and in the case of an anomaly-was also calibrated.

This procedure enabled determination of each response function and geometric factor as documented by Collinson et al. (2012). That is,

$$
G_{i, j, k} \approx \frac{\Delta \theta}{E_{0}^{2}} \sum_{m} \sum_{n} \frac{C_{i, j, k}}{\Phi} E_{m} \cos \phi_{n} \Delta E_{m} \Delta \phi_{n},
$$

where $G_{i, j, k}$ and $C_{i, j, k}$ are the geometric factor and count rate for pixel $i$ at energy step $j$ and deflection step $k, \Delta \theta$ is the polar angle response width, $E_{0}$ is the center of the energy band pass at energy step $j, \phi_{n}$ is the azimuth incidence angle of the beam at step $n, \Delta \phi_{n}$ is the polar angle step size, and $\Phi$ is the incident beam flux (independently measured in the calibration chamber). The geometric factor was calculated for each illuminated pixel, deflection state, and each particle energy/charge tested.

The DxS energy analyzer constant, $k_{\epsilon}$, is calculated per pixel and deflection state as the ratio of the beam $E / q$ to the $V_{E S A}$ centroid. The $V_{E S A}$ centroid is the ratio of the first and second integral moments of the 1D counts vs $V_{E S A}$ curve, which was obtained by summing a 2D $V_{E S A}$ versus azimuth angle counts distribution over azimuth angles. The fractional width, $d E / E$, is calculated as the FWHM of that same $1 \mathrm{D}$ curve, divided by the value of the $V_{E S A}$ centroid.

The DxS deflection analyzer constant, $k_{\alpha}$, is calculated per pixel as the ratio of the applied $V_{D E F}$ at the peak of a 2D counts vs azimuth angle distribution to the product of: (1) the azimuth angle centroid determined as ratio of the first and second integral moments of the $1 \mathrm{D}$ counts versus azimuth angle curve that is obtained by summing a $V_{E S A}$ versus azimuth angle counts distribution over $V_{E S A}$, and (2) the applied $V_{D E F}$ at the peak of the distribution. The azimuth angle response width $(\Delta \phi)$ is calculated as the FWHM of that same 1D curve.

The location and width of the polar angle band pass for each pixel is determined by analyzing the Polar Angle scans. The location is determined as ratio of the first and second integral moments of the counts versus polar angle distribution for each pixel. The width is the boxcar width. This is the ratio of the area under the counts versus polar angle distribution to the peak count rate in the distribution for each pixel. These quantities are largely dependent upon the ESA optics but the widths in particular also vary significantly with sensor operating point.

Measurements of the effective area were obtained from the energy-angle scan as the ratio of the input beam flux to the peak pixel count rate in each $2 \mathrm{D}$ non-deflected $V_{E S A}$ versus azimuth angle counts distribution.

The pixel sensitivity for each DxS is specified by this geometric factor in units of $\mathrm{cm}^{2} \mathrm{sreV} / \mathrm{eV}$. The factor $(\mathrm{eV} / \mathrm{eV})$ is retained so the fractional energy/charge band pass $(d E / E)$ is clearly understood to be included. The ratio of pixel count rate to the geometric factor gives the differential directional energy flux measurement in units of $\mathrm{eV} / \mathrm{cm}^{2} \mathrm{sreV} \mathrm{s}$. 
Fig. 38 Electron calibration chambers used for all DES beam testing and calibration

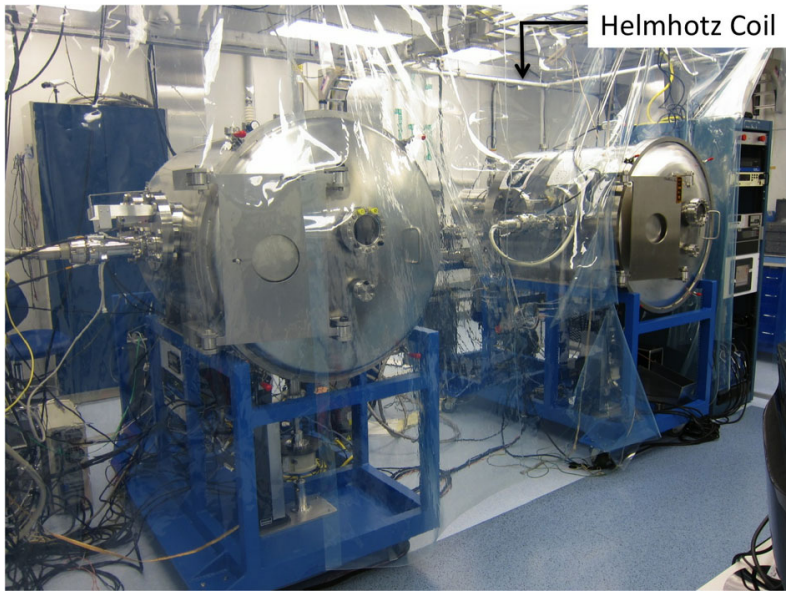

This represents an average over the sampled volume of velocity space, the pixel aperture segment, and the sample integration period.

Many calibrated quantities that characterize the DxS optical performance, such as analyzer constant, the shape and locations of energy and angle band passes, and the geometric factor itself (except for detection efficiency, including pulse height signal loss) are expected to be properties of the electrostatic optics and therefore constant in time across the spectrometer sets and across energy and pixel space. Nevertheless, notable systematic variations are introduced by variations in optical properties at different deflection angles, and by variations around the $180^{\circ}$ pixel space in the angle between an incident particle's velocity vector at MCP impact and the MCP's channel axis, which has an effect on MCP gain and efficiency. Both random and systematic variations are inevitably introduced by imperfections in machining and/or assembly of the optical system that impact the uniformity of response. This has been minimized by pinned mechanical design and careful attention to precision in the optics design and manufacture. Some of these characteristics may change with temperature and, over time, in ways that are not known a priori. These include the output voltage of the high voltage steppers, the MCP pulse height distributions and the A121 thresholds. The MCP pulse generation efficiency is considered unlikely to change with time and/or temperature during the MMS mission.

\subsection{DES Laboratory Calibration}

\subsubsection{DES Calibration Facilities}

The DES detector boards and electronics boxes were calibrated on the bench. DES as a system was calibrated end-to-end under high vacuum with electron beams both prior to and following instrument level environmental testing. The test program utilized two nearidentical electron calibration chambers, each $\sim 0.75 \mathrm{~m}$ diameter and $1 \mathrm{~m}$ long with oil free pumps (Fig. 38). Helmholtz coils around the two chambers reduced the magnetic field strength inside along the path of the electron beam. As a check on the variability of the two systems, calibration measurements were performed on the same instrument sequentially within both chambers. Results for this 'inter-chamber calibration' were within $3 \%$ for all parameters. 
Fig. 39 Magnetic field strength in the vicinity of the vacuum chambers modeled under the effects of Helmholtz coils. The vacuum chambers are represented as rectangles in red on this page, with cryogenic pumps also represented as red rectangles extending above the chambers. The $2 \mathrm{D}$ rectangles actually represent cross sections of the cylindrical chambers and pumps, viewed from above (cylindrical axes lie in the plane of the page, oriented from top to bottom)

Fig. 40 Vacuum chamber cradle shown with DES mounted for testing and RPA/Faraday Cup sensor mounted in the cusp between the two cylindrical DES entrance apertures
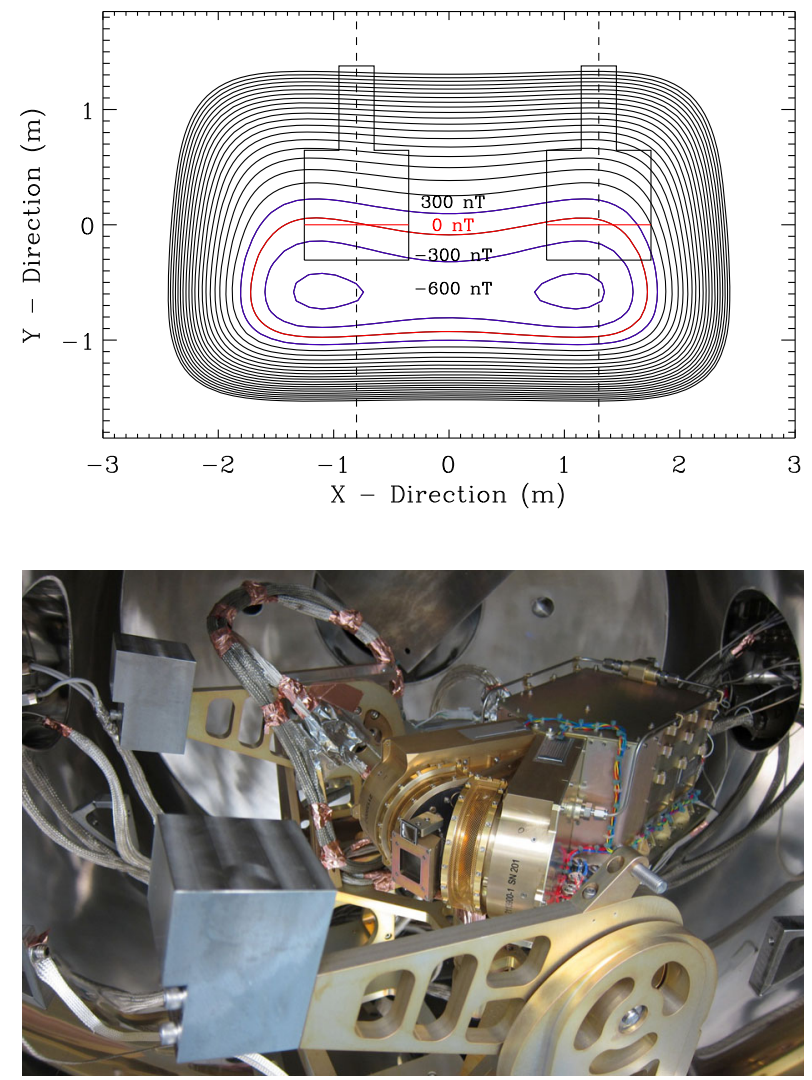

Design of the Helmholtz coils was based on measurements of the magnetic field within the test chambers. This field was dominated by background geomagnetic with components primarily along the N-S aligned cylindrical chamber axes and the local vertical direction. Figure 39 shows modeled magnetic field strength contours in the vicinity of the two chambers with the coils operating. Chamber outlines are shown in red as is the contour of zero magnetic field. The bold blue contours show the $\pm 300 \mathrm{nT}$ level. Typical residual magnetic field intensity along the calibration beam path is predicted to be the order of $100 \mathrm{nT}$, less than $1 \%$ of the naturally occurring geomagnetic field and sufficient to enable electron beam calibration down to $100 \mathrm{eV}$ or less. Quality electron beam trajectories were confirmed at several beam energies using an imaging detector placed at the location of a DES.

For all quantitative DES beam testing, the DES was placed on a cradle in one of the two DES calibration chambers. These cradles feature robotically controlled configurations of azimuth and polar angle rotation and z-axis translation (both in spacecraft coordinates in relation to the instrument) and an additional rotation that enables a flip between sensor head 0 , sensor head 1, and the Faraday cup with respect to alignment with the beam, allowing locating and orienting DES aperture appropriately for beam test sequences. A photograph of DES SN 201 in the cradle is shown in Fig. 40. The two yellow-metal colored $180^{\circ}$ cylindrical DES apertures are evident near center of photo, one almost directly facing the viewer. A square Retarding Potential Analyzer/Faraday Cup (RPA/FC) assembly is mounted on the cradle in between the two sensor heads to provide monitoring of the beam flux at the aperture locations. 
Fig. 41 Normalized threshold sweeps for DES FM205 are shown for Pixel 0 (highest gain) and pixel 7 (lowest gain)
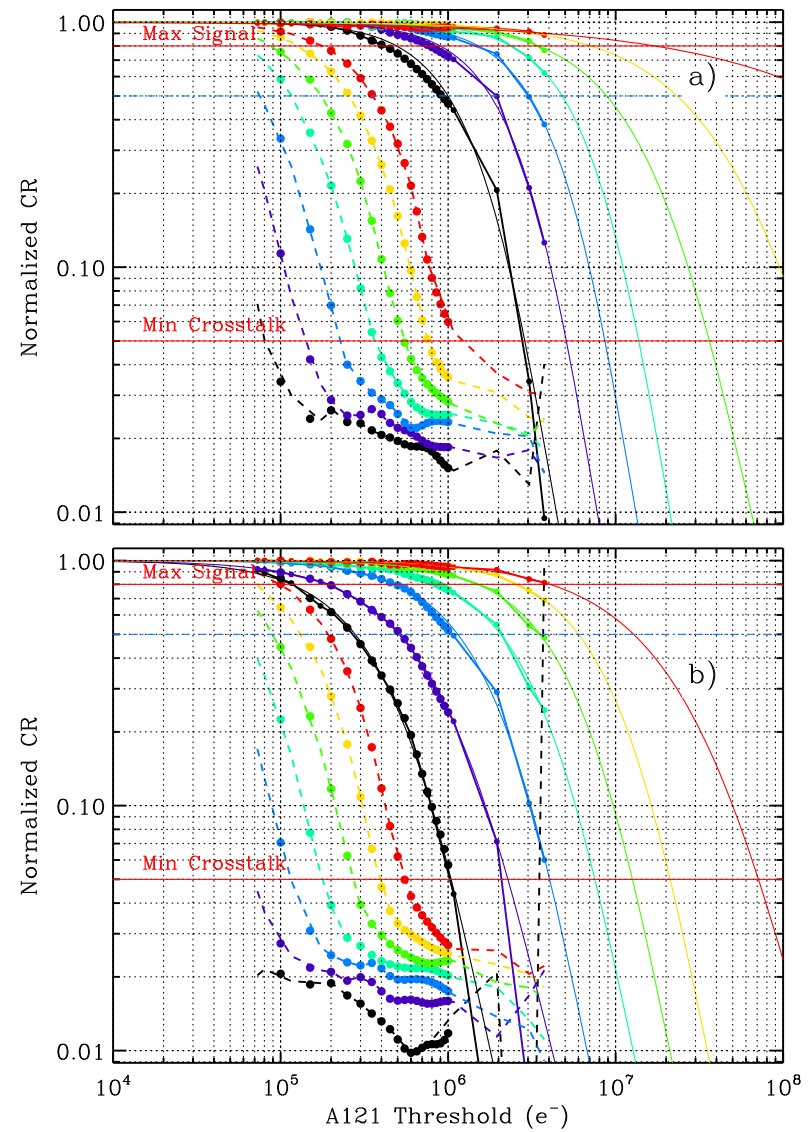

The DES spectrometers were calibrated using Kimball Physics EMG-4212 electron sources. Modifications by Kimball Physics of the electron source controller unit provided a stable beam as low as $10 \mathrm{eV}$, with a uniform, $7 \mathrm{~cm}$ diameter (at the DES aperture) beam possible between $100 \mathrm{eV}$ and $25 \mathrm{keV}$. The flux was measured using a Faraday cup before and after each test to verify beam stability. The Faraday cup was located between the two DES apertures, as illustrated in Fig. 40. Beam profiles were taken on several occasions throughout the calibration program at several energies using a Quantar 2D beam imager to confirm uniformity.

\subsubsection{DES Operating Point Determination}

Figs. 41 through 43 illustrate the process of selecting the operating point for DES Flight Model (FM) 205. Performed for each flight sensor, this ensures all MCPs operate at the same minimum gain target and all detection systems operate with the optimum balance between signal-loss and crosstalk.

Figure 41a shows normalized threshold scans for Pixel 0, which is chosen owing to its systematically high gain relative to the other pixels and so contributes the highest capacitive crosstalk to its nearest neighbor. Pixel 0 and Pixel 1 count rates are plotted as a function of discriminator threshold for seven MCP high voltage values. Similarly, Panel b of Fig. 42, 
Fig. 42 Use of combined normalized threshold sweeps to select MCP operating voltage
Fig. 43 Use of parameters signal loss (curves rising with threshold, plotted on left scale) and crosstalk (curves falling with threshold plotted on right scale) for selecting detector operating point
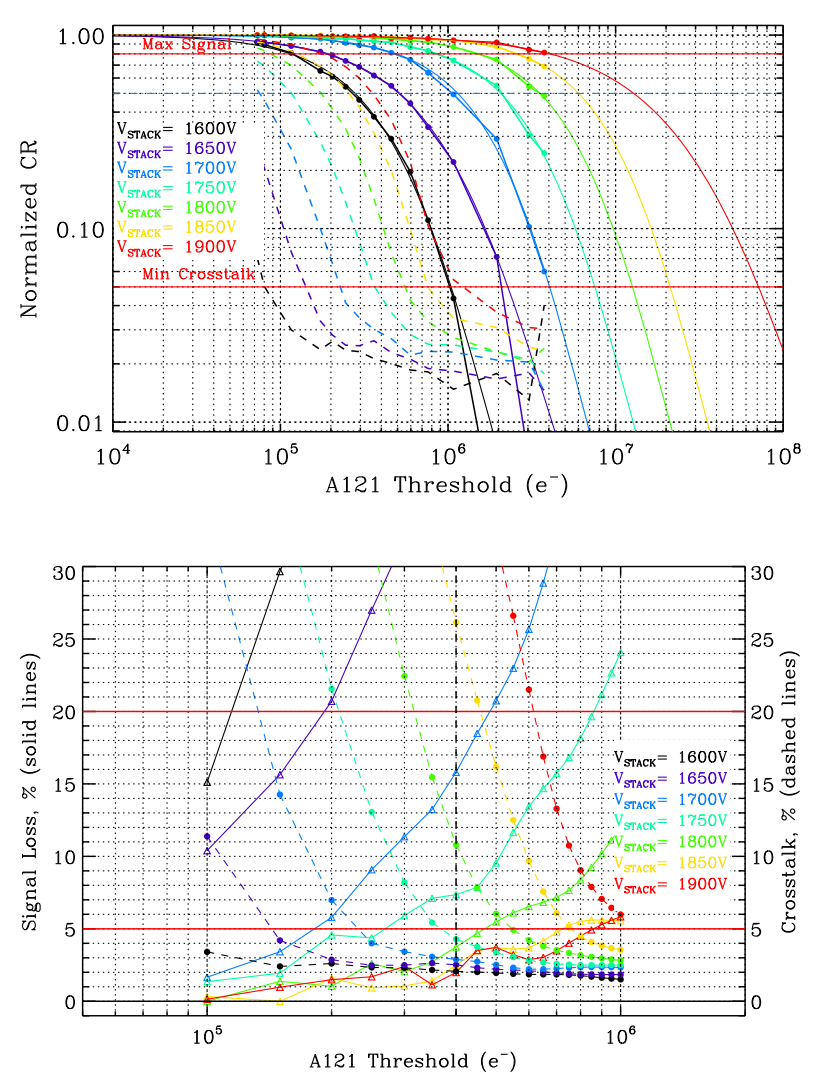

shows threshold scans for Pixel 7, near the center of the array where the gain is lowest. Normalized count rates are plotted for the primary Pixel 7 and the average of its two nearest neighbors (Pixels 6 and 8). Here, with lower gain, the capacitive cross talk is less but the signal loss at a given MCP voltage and threshold (operating point) is larger. Generally, DES detectors display larger gains near the ends of their pixel arrays and smaller gains near the array center due to a systematic variation in the angle between the incident electron velocity vector at impact and the axes of the cylindrical MCP pores. This has not been systematically observed in the DIS sensors.

Figure 42 combines threshold sweeps, taking cross talk curves from the worst-case crosstalk pixel ( 0 in this case) and signal curves from a worst-case signal-loss channel (7 in this case) into a single composite threshold sweep. This illustrates the space within which this DES sensor should operate for optimal performance. For this flight model, the initial MCP voltage selected was that which produced a gain of $2 \times 10^{6}$ at the pixel with minimum gain (7 in this case).

Converting normalized signal rate into signal loss (signal loss $=1$-normalized rate) and re-plotting both the cross talk and signal loss versus discriminator threshold yields the information shown in Fig. 43. Though cross talk and signal loss are displayed for several values of MCP voltage, selection of our MCP voltage $(1750 \mathrm{~V})$ has been already made based on the data in Fig. 42 to ensure the gain target is met. Using the graphs acquired for the chosen MCP voltage, the discriminator threshold was chosen that provided the best trade-off between signal-loss and crosstalk, which in this case is $4 \times 10^{5}$ electrons/pulse. Further 

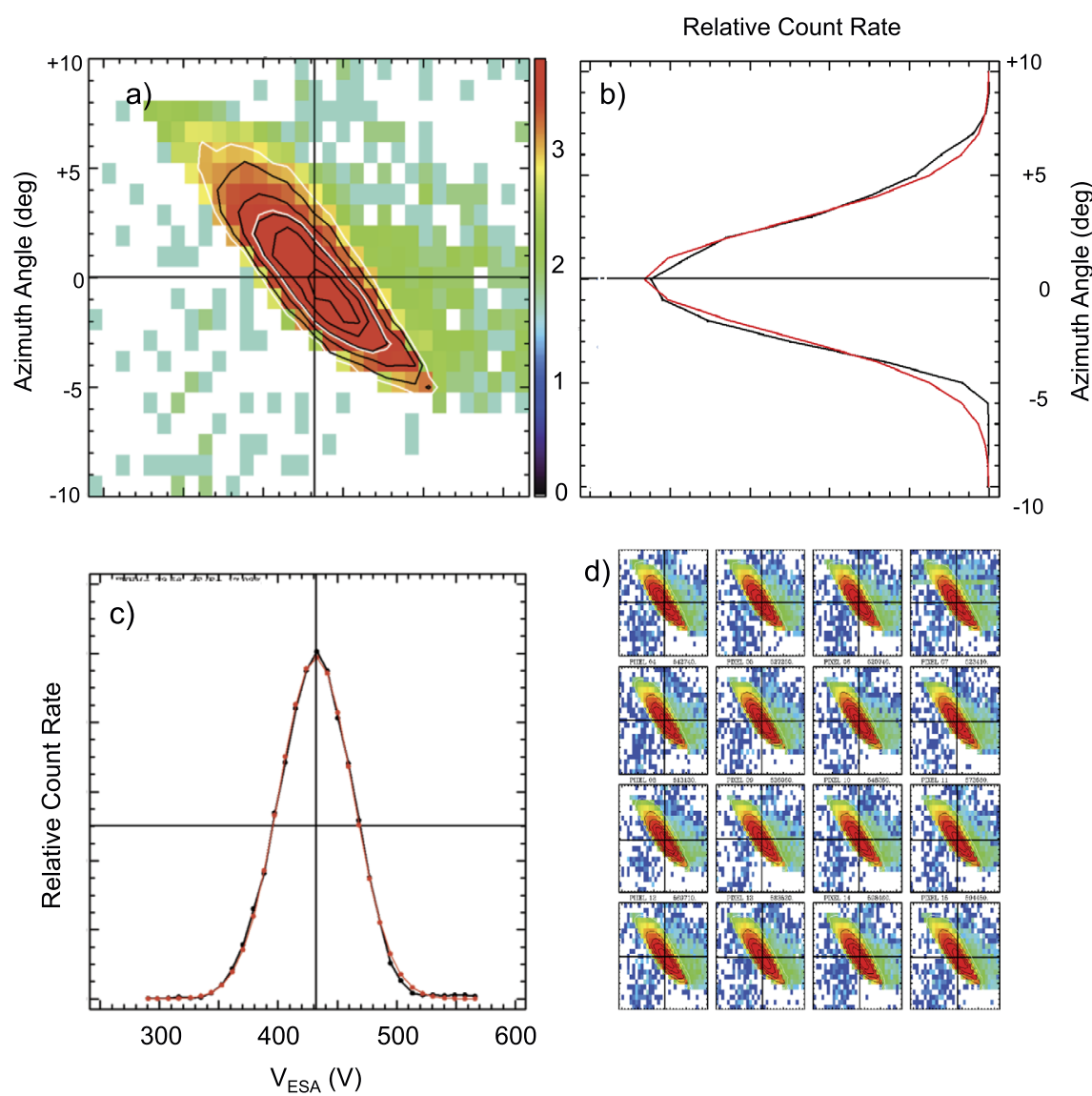

d)

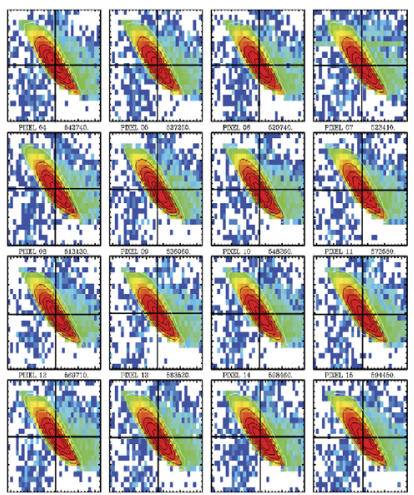

Fig. 44 Typical VESA/azimuth-angle response calibration data, in this case from DES FM Unit 201. In panel (a) the 2D counts distribution is plotted on a logarithmic color scale versus VESA on the horizontal axis and azimuth angle on the vertical axis. Its 1D reduced distributions are plotted versus azimuth angle in panel (b) and versus VESA in panel (c). In both cases, the counts are summed over the second dimension to produce the reduced distributions. Panel (d) contains sixteen such 2D distributions, one each for each of the sixteen anodes in this sensor, illustrating at zeroth order the similarity in the response across pixels

optimization would have been possible if FPI had individually controlled the threshold settings for the 16 A121 devices in each sensor, albeit for considerably increased operational complexities.

This process of selecting the calibration operating point was followed for each sensor on all flight DES units. A similar process will be followed on orbit to evenly maintain the DES operating points in flight.

\subsubsection{DES Energy-Azimuth Angle Scans}

Measurements of the coupled energy/angle band passes were acquired and analyzed across pixel space, at all deflection states, and at multiple energies in the range $100 \mathrm{eV}$ at the lowest to $30 \mathrm{keV}$. Examples for a single deflection state are shown in Fig. 44. There, we show the 2D angle/energy band pass (Fig. 44a) and the two corresponding 1D angle (Fig. 44b) and 
Fig. 45 Polar angle scan for DES FM202. The count rates for different pixels are shown in different colors with key on the right. The total counts, summed over pixels, is also plotted, the dark grey curve riding on top of the colored curves

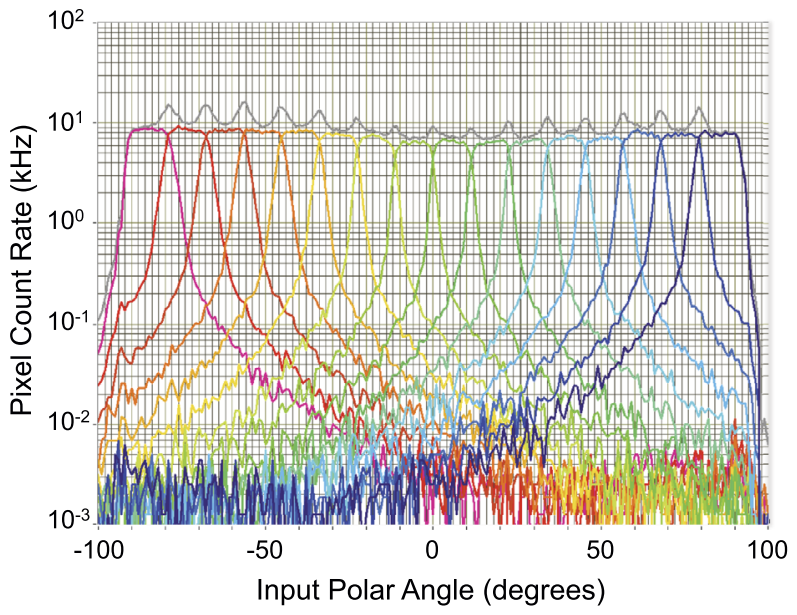

energy (Fig. 44c) distributions that result from summing over the second dimension. Fits to these distributions were performed and angle and energy band pass centers and widths derived. Count distributions like that shown in Fig. 44a were summed in accordance with Eq. (5) and combined with independently measured beam flux and polar angle response to yield the geometric factors for every pixel at several different energies. Figure $44 \mathrm{~d}$ shows a collection of 2D distributions like that shown in Fig. 44a, one for each pixel. The uniformity of response was found to be excellent.

\subsubsection{DES Polar Scans}

An example polar angle scan for DES FM206 is provided in Fig. 45; these provide the $\Delta \theta$ factor in Eq. (5), in addition to the pixel polar angle look directions and measures of cross talk among pixels. The count rate for each pixel is plotted in color with the sum plotted in grey. The peaks in total rate appear at the junctions and are attributable to pulse spreading between the MCP and the anode, which often yields two pulses for a single event. Polar angle scans can also be used to identify noisy pixels if the count rate does not fall off as quickly as expected. The laboratory polar responses were modeled as described in Gershman et al (submitted), including effects such as the spot size on the MCP input face, the scattering of primary and secondary electrons from surfaces of the ESA and MCP, and MCP pulse spreading. This model will be used when monitoring and correcting for performance trends in flight.

\subsubsection{DES Saturation Characterization}

DES has two primary sources of count rate saturation, (1) MCP saturation depends on the count rate per unit MCP area and is paralyzable, (2) sensing amplifier saturation is nonparalyzable with a dead time near $100 \mathrm{~ns}$. Measurements of the MCP saturation are shown in Fig. 46.

First, an electron beam narrow in energy and angle was directed at the DES optics aperture while the ESA and deflection voltages were set to pass the beam to the detector; the count rate was recorded as the incident flux was increased. MCP saturation depends upon resistance (e.g., lower resistance MCPs count faster) and also on the rate per unit area (e.g., 


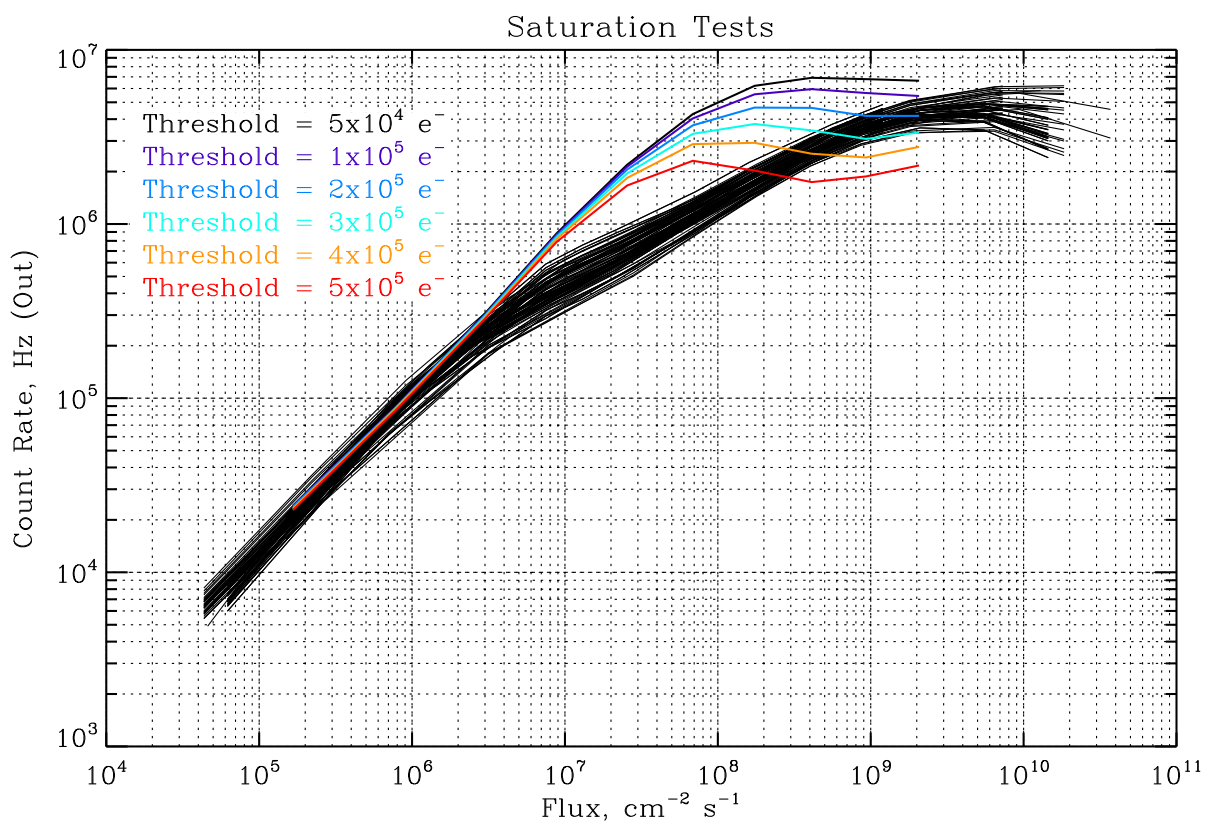

Fig. 46 Saturation properties of DES. Data shown are from two separate sets of measurements. The black curves represent measurements made with 10 different DES flight units (20 sensors)

size of the signal spot on the MCP). The inflection in the set of black curves ( 20 sensors) is attributed to spot size localization imposed by the optics. A second set of saturation tests on the MCP/Anode/Amplifier assemblies were performed without the optics, which resulted in a larger spot on the MCP surface. These tests, performed at several discriminator thresholds, are shown with the colored curves in Fig. 46. For a threshold of $4 \times 10^{5}$ electrons per pulse, the count rate increases linearly with flux until it begins to deviate from linear near $100 \mathrm{kHz}$ and saturates near $3 \mathrm{MHz}$.

\subsubsection{DES UV Rejection}

The ratio of the count rate observed to the rate of photons entering the aperture is a measure of Ly- $\alpha$ sensitivity and is required to be smaller than $10^{-8}$. ESA surfaces were coated with an Ebanol-C layer, significantly decreasing potential reflections due to its high UV absorption. In the beam chamber a resonant UV Kr lamp (Opthos Instruments, Inc.) was used to produce a uniform photon flux across the DES entrance aperture with two resonant lines (116.5 and $123.6 \mathrm{~nm}$ ) close to the Ly- $\alpha$ wavelength $(121.6 \mathrm{~nm})$. A UV AXUV-100GLA was used to estimate the photon flux illuminating the aperture. Conversion of the current measured through the diode to the photon flux used the published quantum efficiency of the diode, near 1.78 electrons/photon at these wavelengths. Photon flux was controlled by the power applied to the lamp, producing $3 \times 10^{11}-4 \times 10^{11}$ photons $/ \mathrm{cm}^{2} \mathrm{~s}$, the approximate flux of solar Ly- $\alpha$ at $1 \mathrm{AU}$, with a lamp power of $\sim 40 \mathrm{~W}$. The DES was rotated in two dimensions to illuminate all pixels with a range of azimuth angles. Figure 47 (DES FM206) shows that the rejection ratio to the input photons flux remains at the level of $10^{-11}$ three orders of magnitude lower than required ratio $10^{-8}$. 


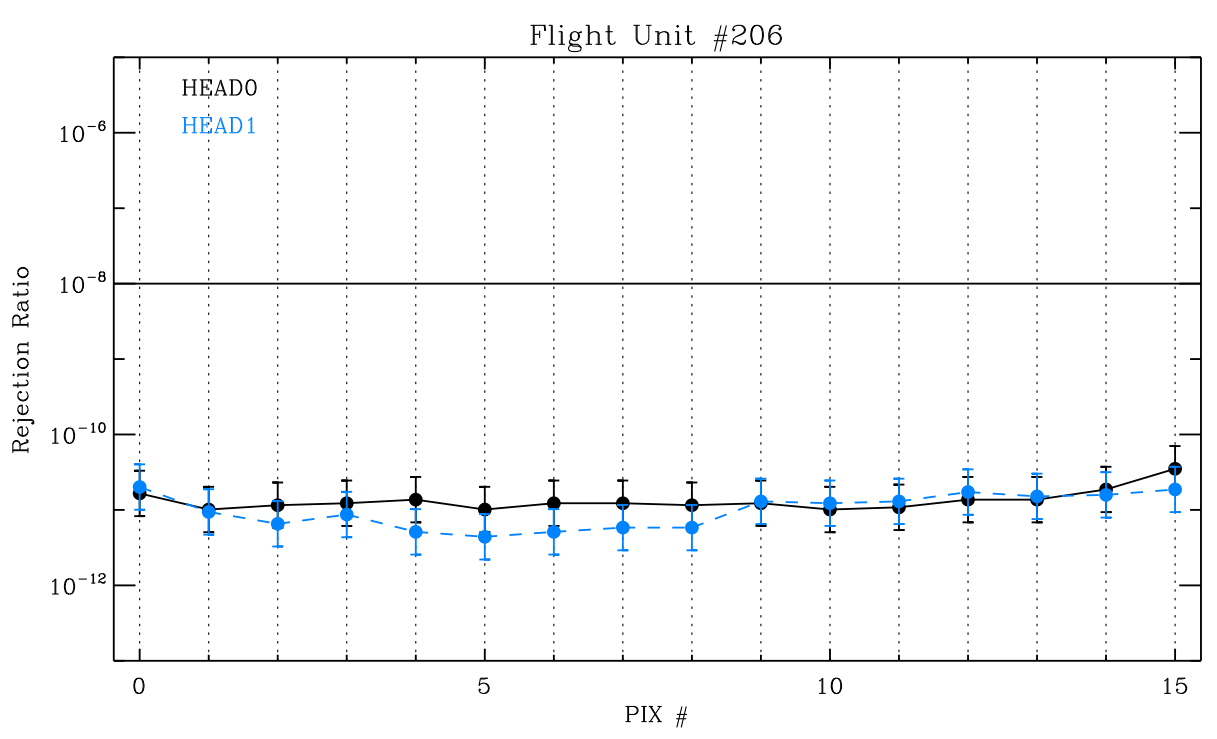

Fig. 47 Results of UV testing of DIS FM 206

Fig. 48 Measurements of DES geometric factor for all sensors, pixels, and deflection states are shown as red lines. The solid line represents the mean value and the two dashed lines show one standard deviation above and below that value

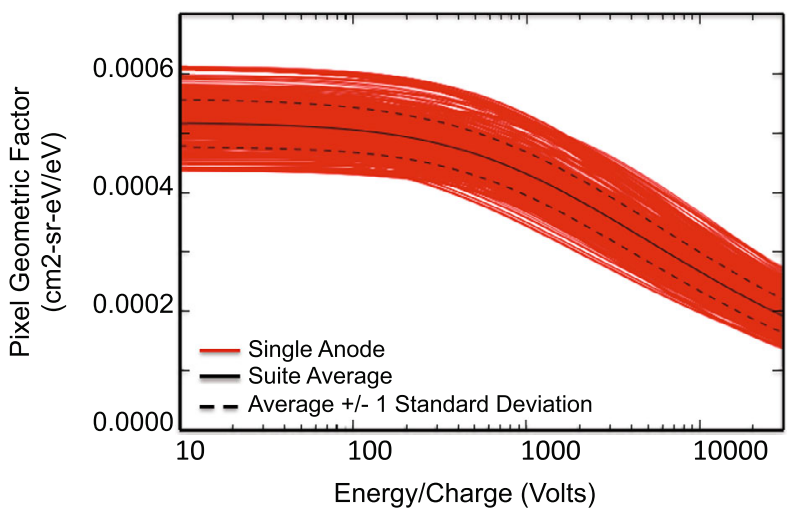

\subsubsection{Summary of DES Laboratory Calibration Results}

Table 4 summarizes DES performance parameters and their variability as measured for the 16 units (32 sensors). The factors $k_{\epsilon}$ and $k_{D E F}$ are the analyzer and deflection constants and are defined in the table heading. The deflection state conventions are with respect to the sensor, where "Down" means viewing toward the plane of the MCP and "Up" means viewing away from the MCP plane. The energy at which the geometric factor is cited is $1 \mathrm{keV}$. As expected, there is significant energy dependence in the geometric factor owing to variations in MCP detection efficiency with electron energy (Bordoni 1971); this variability is shown in Fig. 48. 


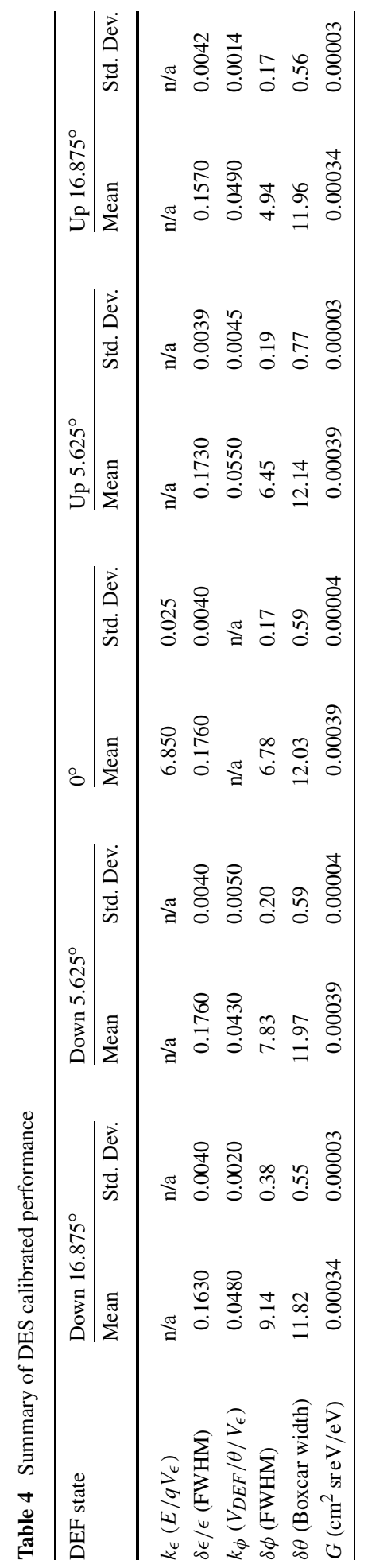




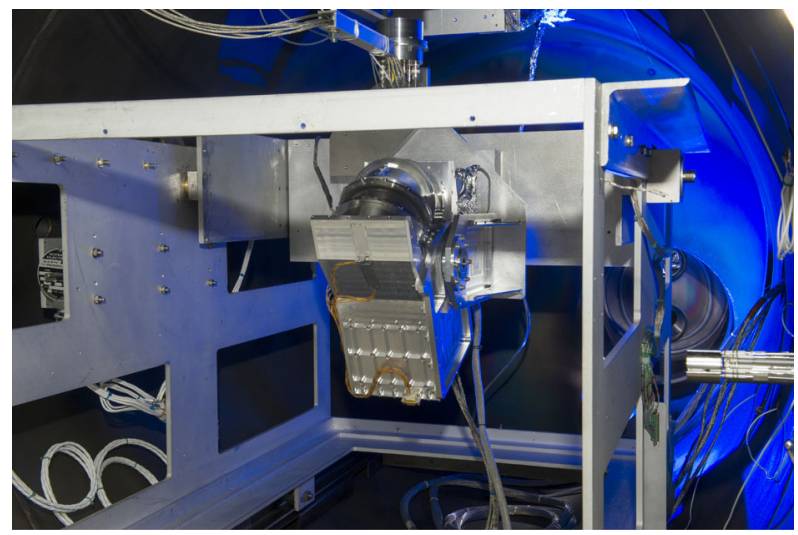

Fig. 49 The DIS ETU in the calibration chamber at NASA's Marshall Space Flight Center. The system included (1) large oil-free vacuum chamber capable of reaching pressures less than $10^{-7}$ Torr, (2) an ion source providing a steady mono-energetic, mono-directional positive ion beam with energies/charge ranging from $10 \mathrm{eV}$ to $30 \mathrm{keV}$ and fluxes in the range of $10^{3}$ to $10^{8} \mathrm{~cm}^{-2} \mathrm{~s}^{-1}$, (3) support fixtures allowing 2D orthogonal rotation of each sensor aperture under the ion source, (4) a Retarding Potential Analyzer (RPA) for ion source diagnostics $\leq 4.5 \mathrm{keV}$, (5) DIS Ground Support Equipment (GSE) for control and data acquisition; and (6) synchronized control/data collection between the GSE and chamber devices for real-time viewing and analysis

\subsection{DIS Laboratory Calibration}

\subsubsection{DIS Calibration Facility}

The DIS flight units were calibrated at the NASA MSFC Low Energy Electron and Ion Facility (LEEIF), which is specifically designed for the testing and calibration of particle detectors (Biddle and Reynolds 1985). Figure 49 is a photograph of the DIS ETU mounted in the vacuum chamber on a mechanical rotation fixture directly below the vertical ion beam. The moveable RPA (top center of the photo) is used to determine the beam flux. The mechanical polar angle range of $\pm 110^{\circ}$ moved the DIS through its array of 16 anodes. The mechanical azimuth angle range of $-21^{\circ}$ to $26^{\circ}$ moved the DIS through the electrostatic deflection angles.

A Kaufman Thruster ion source produced ions by electron impact ionization and accelerated them electrostatically to the required energy/charge (up to $50 \mathrm{keV} / \mathrm{q}$ ). Typical operating current densities were near $0.05 \mathrm{pA} / \mathrm{cm}^{2}$ and ion beam temperatures ranged from $<1 \%$ of drift energy at $3 \mathrm{keV}$ to $30 \%$ at $10 \mathrm{eV}$.

Prior to testing, the ion source optics and RPA diagnostics were validated using a cylindrical ESA of $0.5 \%$ energy and $0.25^{\circ}$ angle resolution (Valek 2001). Two RPAs were used, one to measure energy/charge up to $1.5 \mathrm{kV}$ and another to measure energy/charge up to $4.5 \mathrm{kV}$. Tests were simultaneous over the overlapping energy ranges of each device to calibrate the admitted particle energy and confirm agreement between the devices. Further, during calibration of DIS FM10, the RPA was cross-calibrated against an Absolute Beam Monitor (ABM) (Funsten et al. 2005) provided by Southwest Research Institute (SwRI). The beam fluxes measured by the RPA and the ABM device agreed to within $6 \%$.

Incident beam energy/charge is determined by fitting a Gaussian to the negative first derivative of an RPA curve, $-d I_{F C} / d V_{R P A}$ and was observed to be stable with time. The RPA was moved into the beam approximately $20 \mathrm{~cm}$ above the DIS aperture at the beginning, 
Fig. 50 Example results from DIS detector characterization
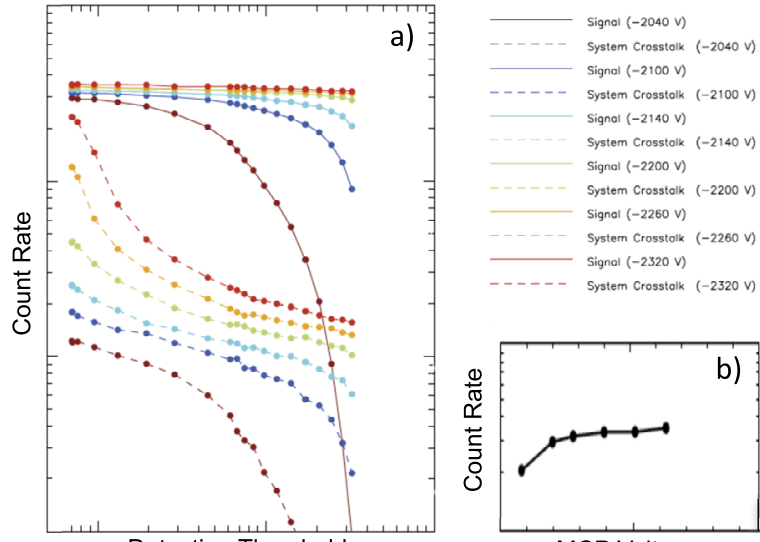

MCP Voltage

middle, and end of each procedure and the beam current measured at $V_{R P A}=0$. The mean value of these three measurements is used in combination with the Faraday Cup Effective Area of $2.99 \mathrm{~cm}^{2}$ to compute the beam flux.

Molecular nitrogen gas was used as the bleed gas to calibrate the DIS flight sensors although protons will comprise the majority of ions in flight. The MCP gain is expected to be $\sim 2 \mathrm{X}$ larger under proton excitation in flight, as compared with the molecular nitrogen calibration beam, based on Fig. 2 of Oberheide et al. (1997) and Fig. 1 of Furuya and Hatano (2002). The detection efficiency should also be larger for protons than for molecular nitrogen, based on Fig. 3 of Oberheide et al. (1997). Both effects will be quantified with inflight commissioning/calibration procedures.

\subsubsection{DIS Operating Point Determination}

Figure 50 shows the process of selecting the operating point for DIS Flight Model 14 sensor head 1. Figure 50a shows normalized threshold scans, where count rates in Pixel 7 and the average of those in Pixels 6 and 8 are plotted versus the nominal value of the discriminator threshold. As with DES, the rates in the primary pixel (in this case, Pixel 7) are used to ascertain the MCP gain and the rates in neighboring pixels determine cross talk characteristics. The MCP operating point was finalized using a single MCP gain plateau curve (Fig. 50b) acquired for each pixel by fixing the threshold near $4 \times 10^{5}$ electrons/pulse and sweeping MCP voltage while recording the count rate. The MCP operating point voltage was fixed $\sim 50-100 \mathrm{~V}$ higher than that at which the count rate in the lowest gain pixel plateaued. In the case of DIS FM14 sensor head 1, the MCP operating voltage was chosen to be $-2250 \mathrm{~V}$.

\subsubsection{DIS Energy-Azimuth Angle Scans}

The energy-azimuth angle procedure was conducted for each pixel by mechanically scanning the beam over the full range of deflected FOVs. The energy/angle stepper table was tailored to the energy/charge under calibration and the five deflection states: $-16.865^{\circ}$, $-5.625,0.0^{\circ},+5.625^{\circ}$ and $+16.875^{\circ}$ and resulted in $2 \mathrm{D}\left(V_{E S A}\right.$, Azimuth angle) count rate maps at each deflection.

Figures 51 and 52 show the output of this procedure for Pixel 8 on DIS FM014, Sensor 0 and Sensor 1, respectively. Figures 51a and 52a contain five 2D maps of counts versus $V_{E S A}$ 

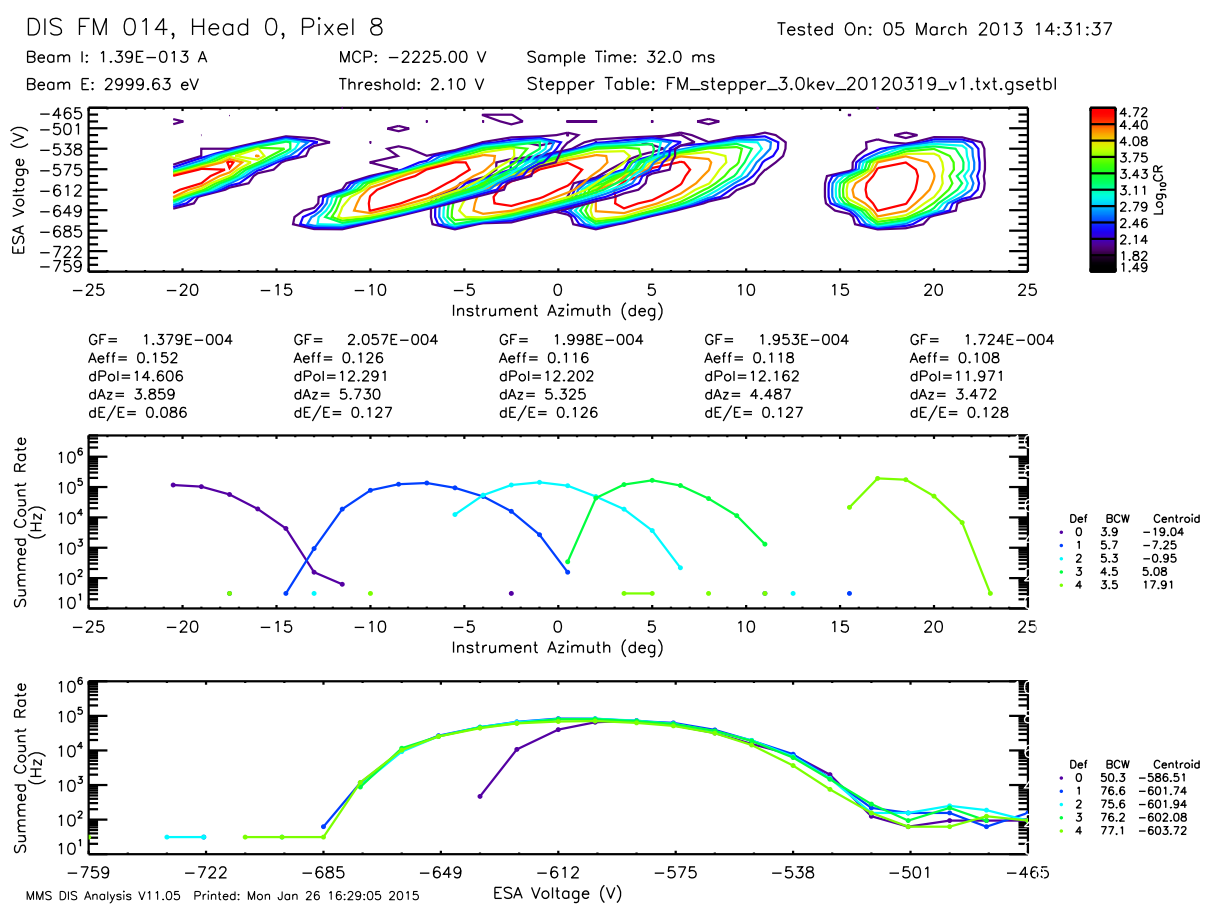

Fig. 51 Near real time graphical and tabular output from the DIS energy/angle response procedure is presented in the case of FM 14, Sensor 0, Pixel 8. Panel a (top) shows the 2D VESA/Azimuth Angle response at five deflection states, nominally centered at $-16.875^{\circ},-5.625^{\circ}, 0.0^{\circ},+5.625^{\circ}$, and $+16.875^{\circ}$. The contours are color coded according to the $\log 10$ of the count rate as defined in the color bar at the right of Panel a. Panel b (center) shows 1D azimuth angle response obtained by summing the distributions in Panel a over VESA and Panel c (bottom) shows 1D VESA response obtained by summing the distributions in Panel a over azimuth angle. The lines in Panels $\mathbf{b}$ and $\mathbf{c}$ are color coded according to deflection state, most evident in Panel b. Between Panels a and b, provisional (near real time) values of the geometric factor ("GF"), effective area (" $A_{e f f}$ "), polar angle band pass width (" $d_{P o l}$ "), azimuth angle band pass width (" $d A z$ ") and energyband pass width (" $d E / E ")$ are tabulated

and mechanically controlled angle between the beam axis and the plane perpendicular to the tophat symmetry axis. The energy/angle sweep uses nominal values for $V_{D E F} / V_{E S A}$ of 0.84 and 0.28 . Summing these maps over the $V_{E S A}$ dimension provides the $1 \mathrm{D}$ azimuth angle distributions shown in Figs. 51b and 52b, while summing over the azimuth angle distributions provides the 1D $V_{E S A}$ distributions shown in Figs. 51c and 52c. These 1D distributions were used to compute performance parameters for each pixel and deflection state, including the locations and widths for both $V_{E S A}$ and azimuth angle band passes.

The Sensor 0 (Fig. 51) 2D counts distributions are highly elongated at negative azimuth angles and more nearly circular at positive azimuth angles. Those in Sensor 1 (Fig. 52) are more elongated at positive azimuth angles and more circular at negative azimuths. This effect is common to DES and DIS and predicted by ray tracing. It affects the derived parameters primarily through a smaller width for the azimuth angle band pass. Neither the energy/charge band pass nor the geometric factor is strongly affected. Note also the truncation of the 2D count rate distributions and 1D counterparts at large negative angles. These truncations reflect the limited range of the calibration chamber's azimuth rotation stage. 


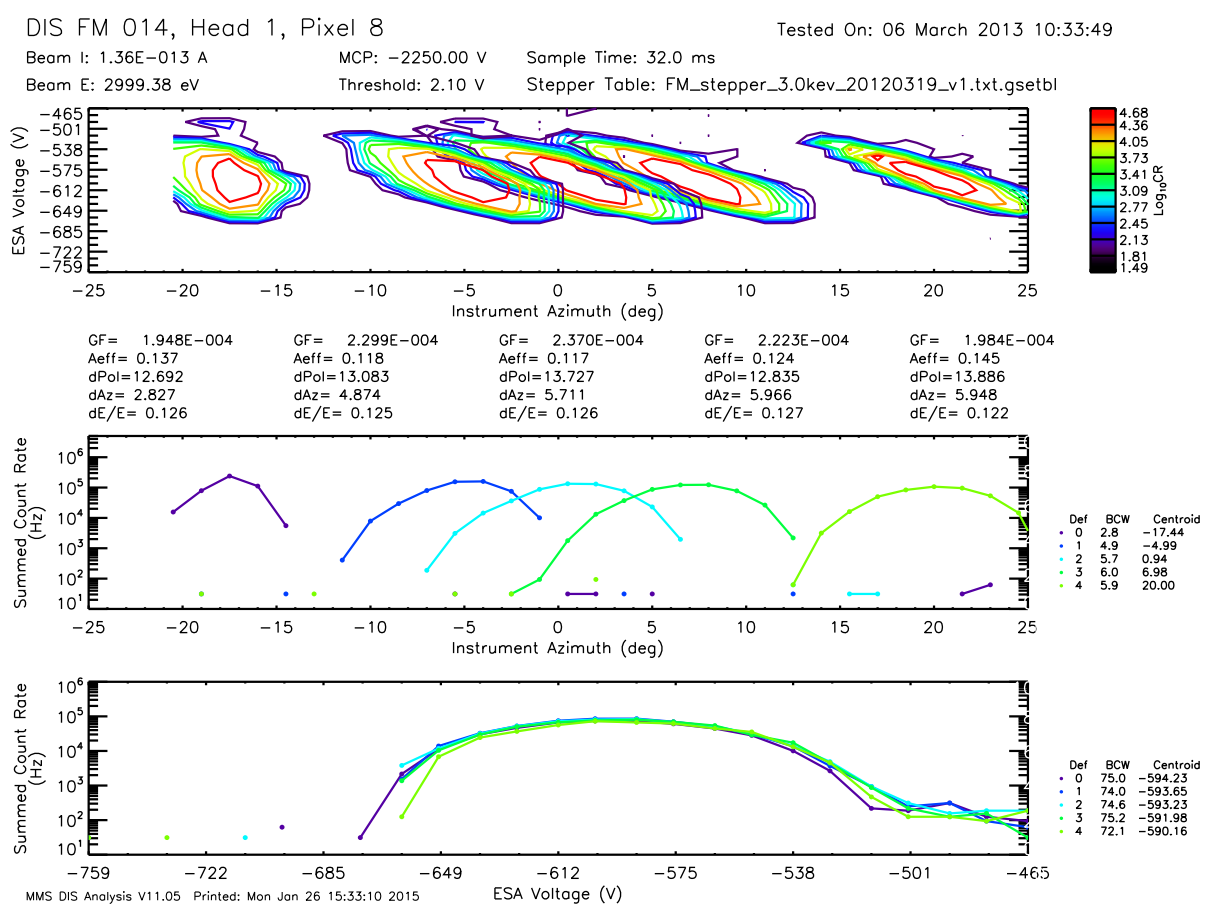

Fig. 52 Same as Fig. 51, except for the case DIS FM 14, Sensor 1, Pixel 8

\subsubsection{DIS Polar Angle Response}

The location and width of each pixel's polar angle band pass was determined as the spectrometer was mechanically rotated from anode to anode and over each polar FOV. Figures 53 and 54 show the response for DIS FM004 Sensor 1, for deflection angles of -16.875 and +16.875 , respectively. Note the difference in the widths of the angle distributions $\left(11^{\circ}\right.$ versus $15^{\circ}$ ). This is observed for all DIS (not DES), at all energies/charge studied, and could be related to enhanced cross talk due to the scattering of secondary electrons near the surface of the MCP. The pulse height distributions of scattered events are different from those of primary events in DIS, whereas they are the same in the case of DES. This may be because we are always observing electron pulse height distributions in DES, whereas primary DIS events are ions and secondary DIS events are electrons. The azimuth angle dependence of this variation is not understood but is thought to be associated with the different radial footprints of the calibration beam on the MCP and differences in local electron transport near the MCP surface.

\subsubsection{Summary DIS Laboratory Calibration Results}

Table 5 summarizes DIS performance parameters for each of the five flight deflection settings. The values and uncertainties listed represent mean values and standard deviations based on independent measurements across 16 DIS $\times 2$ sensors $\times 16$ pixels. The heavily truncated counts distributions discussed in the context of Fig. 51a are not included in the calculations. The factors $k_{\epsilon}$ and $k_{D E F}$ are the analyzer and deflection constants and are defined 

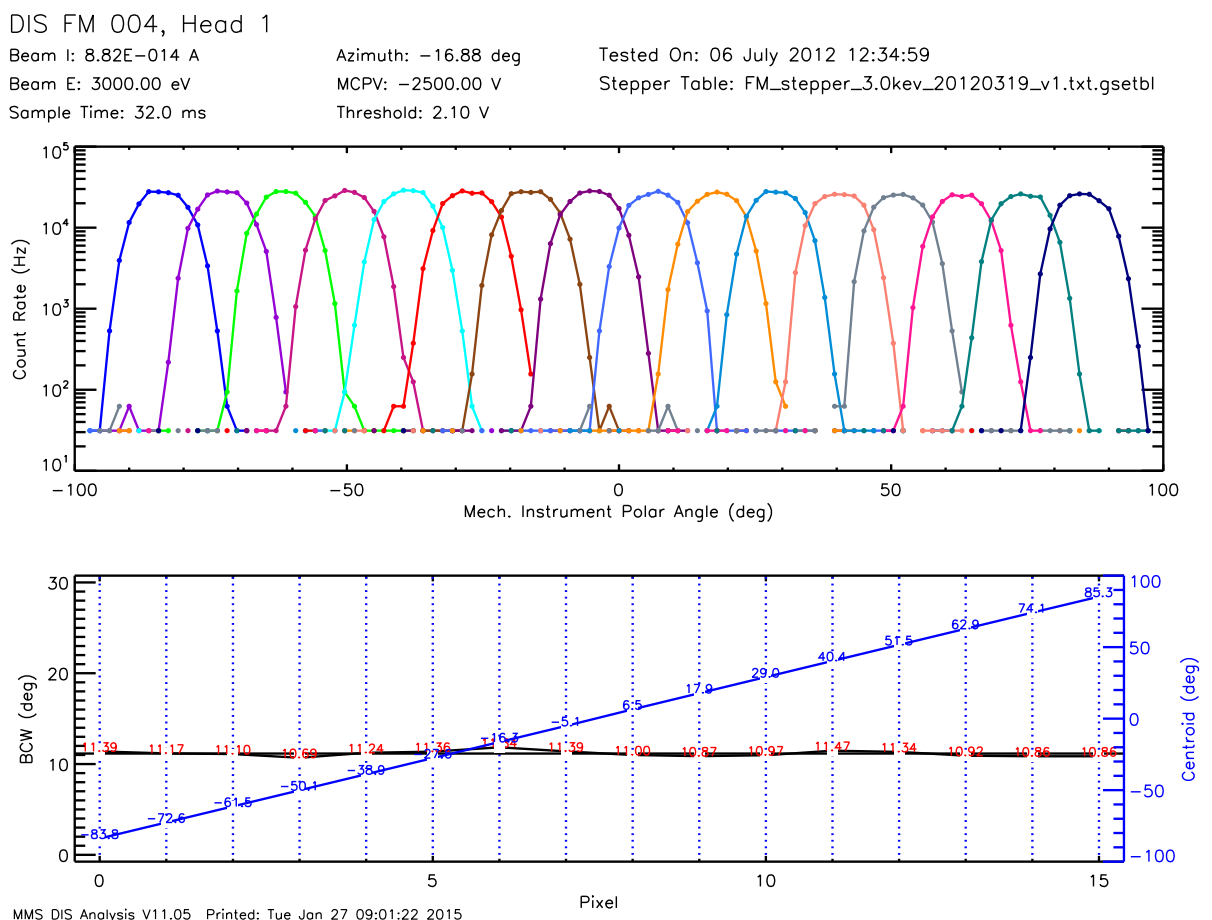

Fig. 53 Near real time output from the DIS polar angle response procedure is presented in the case of FM 04, Sensor 1 , for the $-16.875^{\circ}$ deflection state. Panel a (top) shows the count rates of each pixel plotted versus FPI polar angle. The lines are color coded according to pixel number. Panel b (bottom) shows centroid (axis on right) and width (axis on left) of each pixel's polar angle band pass plotted versus pixel number

in the table heading. The deflection state conventions are with respect to the sensor, where "Down" means viewing toward the plane of the MCP and "Up" means viewing away from the MCP plane. The decrease in the width (df) of the azimuth angle distribution as the look direction proceeds from downward to upward is evident as are the relative uniformities of the width of the energy band pass and the geometric factor. The geometric factor for DIS as measured during laboratory calibration is lower than the required $>5 \times 10^{-4} \mathrm{~cm}^{2} \mathrm{sreV} / \mathrm{eV}$ (Table 1). These measurements, combined with ion optics simulations, indicate a lower than expected MCP efficiency by a factor of $\sim 2$. On-orbit data will be used to resolve this discrepancy through comparisons of FPI-calculated densities with those estimated from lower hybrid wave frequencies.

\section{FPI Flight Operations}

In orbit (Fig. 56) FPI operates in several different modes. It operates in Fast Survey mode when the spacecraft is within the Science Region of Interest (ROI), nominally defined as the high altitude half of each orbital period; and operates in Slow Survey mode or one of several calibration modes between $L=7$ and the beginning of the next ROI; and operates in a low voltage Engineering Mode below $L=7$ to suspend operations when traversing the radiation belts. 
DIS FM 004, Head 1

Beom I: $8.82 \mathrm{E}-14 \mathrm{~A}$

Beom E: $3000.00 \mathrm{eV}$

Somple Time: $32.0 \mathrm{~ms}$
Elevotion: $16.88 \mathrm{deg}$

MCPV: $-2500.00 \mathrm{~V}$

Threshold: $2.10 \mathrm{~V}$
Tested On: 06 July 2012 12:34:52

Stepper Toble: FM_stepper_3.0kev_20120319_v1.txt.gsetbl
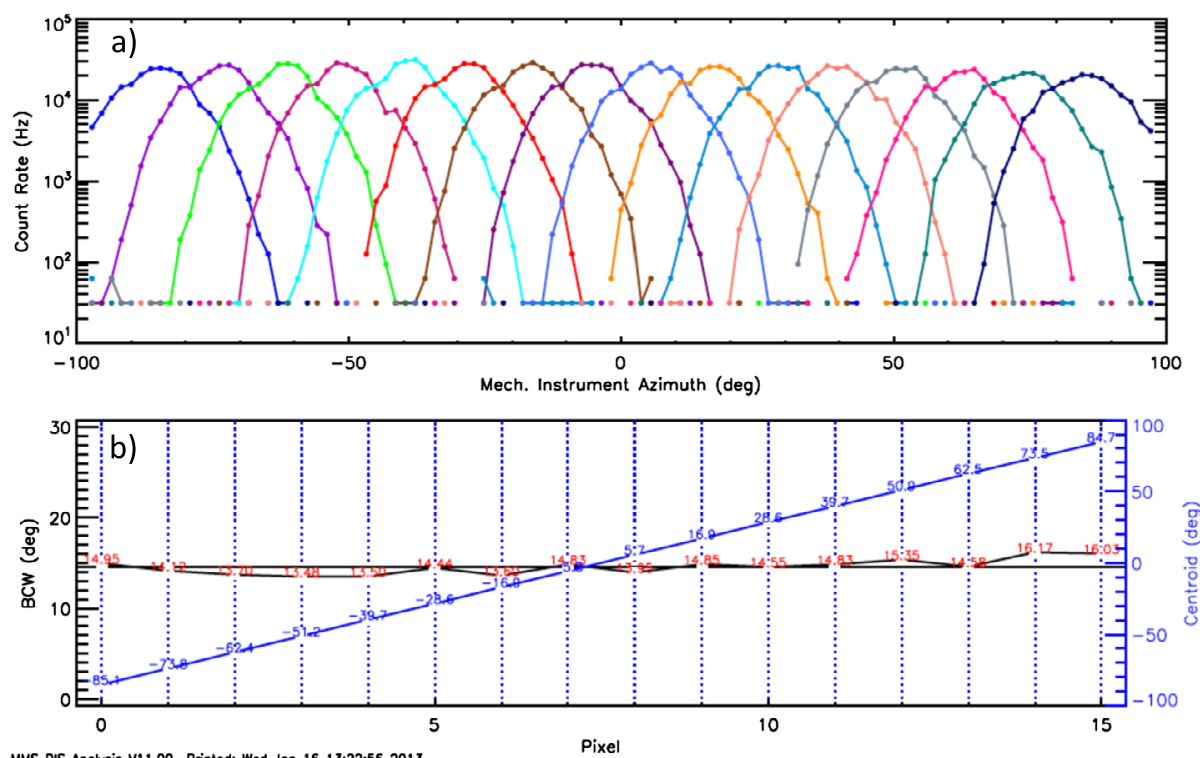

Fig. 54 Near real time output from the DIS polar angle response procedure is presented in the case of FM 04, Sensor 1 , for the $+16.875^{\circ}$ deflection state. Panel a (top) shows the count rates of each pixel plotted versus FPI polar angle. The lines are color coded according to pixel number. Panel b (bottom) shows centroid (axis on right) and width (axis on left) of each pixel's polar angle band pass plotted versus pixel number

In Fast Survey mode, all spectrometers are stepped at their fastest rate, with DES running at $30 \mathrm{~ms}$ and the DIS at $150 \mathrm{~ms}$ for a full Burst sky map at 32 energies and 32 azimuth angles. In this mode, sampling rates are much faster than the spacecraft spin. A Fast Survey data product, reported every $4.5 \mathrm{sec}$, is constructed by de-spinning-performed in the IDPUand summing individual Burst sky maps.

In Slow Survey mode, a single DES and DIS unit are used in conventional spin scan mode to generate a the full sky map every spin, by summing three spins of data for downlink. The specific unit used for Slow Survey is rotated every orbit to balance the observing load across the full suite of spectrometer units.

FPI inflight operational sequences are implemented in the form of Absolute Time Sequence (ATS) commands, which designate when planned activities are to be executed in the context of each spacecraft's activities. All activities are performed in accordance with the FPI mode-switching diagram provided in Fig. 55. Elements in the ATS may take the form of individual commands, Relative Time Sequences (RTS) or Macros. A RTS is a group of commands that includes a relative specification of the delay introduced between each pair of commands in the sequence. A Macro is a group of commands that can be executed rapidly and sequentially with a reliable, fixed delay between commands. The use of RTS and Macros enables FPI to execute the vast array of command sequences necessary to maintain stable. Command macros are generated and managed by means of a suite of Interactive Data Language (IDL) tools designed to facilitate human review and revision. Macros are version- 


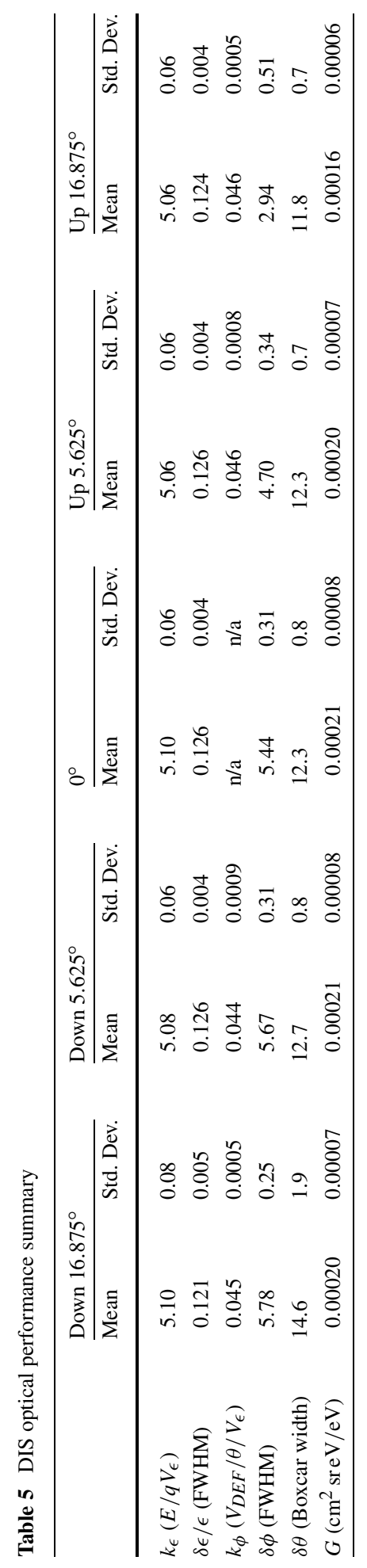


Fig. 55 FPI IDPU state diagram showing modes of operations and permitted mode transitions

Fig. 56 Orbital progression of routine FPI activities

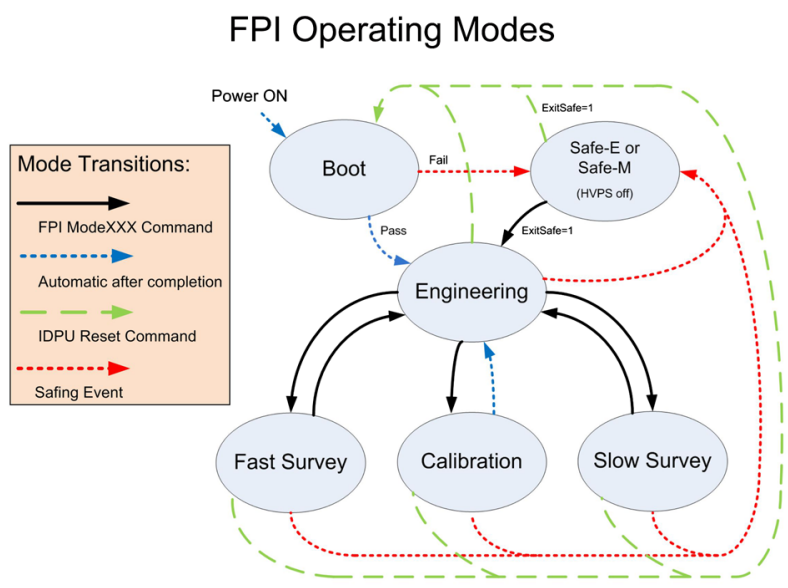

MMS/FPI Nominal Operations

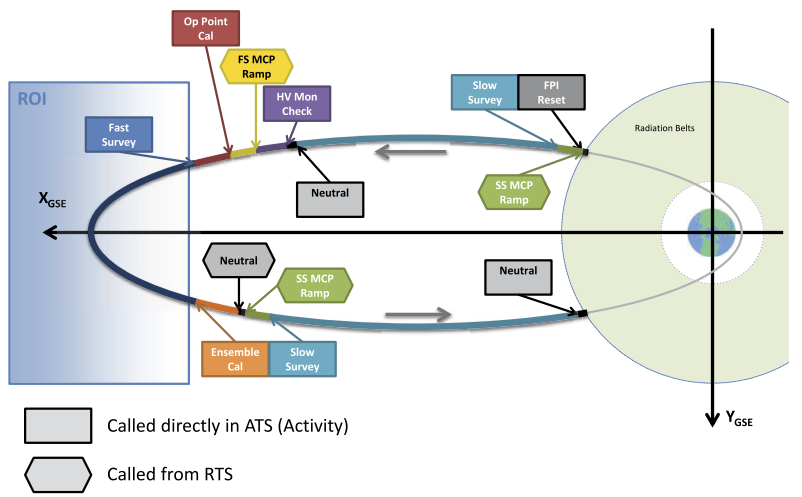

controlled, stored within the CIDP Mass Memory Module, and organized into functional groups. A typical orbit in the life of FPI is illustrated in Fig. 56.

FPI Reset FPI executes a reset upon exiting the inner magnetosphere, at $L=7$, to mitigate corruption of system settings by radiation-related single event upsets. The procedure performs an IDPU soft reset called from the ATS, followed by execution of the FPI Neutral Macro. The FPI operating table chosen is based on the temperature of FPI and is selectable via a parameter in the IDPU reset command.

Neutral The Neutral state ends science operation and ramps down the spectrometer HVPS to a safe state. The command sequence consists of entry to engineering mode, disabling the bulk power supply, and finishes with a soft reset of the spectrometer.

Ramp to Slow Survey The Ramp to Slow Survey activity configures one quadrant of FPI (one DES and one DIS) to ramp up the MCPs and enter Slow Survey data collection. Though all spectrometers remain powered during Slow Survey, only one of the four dual spectrometers pairs has its high voltage enabled. 
Table 6 Voltage settings for HV monitor check for DES and DIS

\begin{tabular}{lcccc}
\hline DES voltages & & & \multicolumn{2}{l}{ DIS values } \\
\cline { 5 - 5 } Low range & High range & & Low range & High range \\
\hline 0.1 & 45 & 0.1 & 55 \\
0.5 & 50 & 0.5 & 60 \\
1.0 & 55 & 1 & 65 \\
5.0 & 100 & 5 & 100 \\
10.0 & 500 & 10 & 1000 \\
30.0 & 1000 & 30 & 3000 \\
50.0 & 3000 & 60 & 5000 \\
- & 5000 & - & 5600 \\
- & 4000 & - & 4000 \\
- & 2000 & - & 2000 \\
& 0 & & \\
\hline
\end{tabular}

Fast Survey The Fast Survey activity sets the integration time to $195 \mu$ s and $1 \mathrm{~ms}$ for DES and DIS, respectively. A snapshot of the instrument configuration (register settings, operating tables, etc.) is recorded prior to entry to Fast Survey mode. Nominally, an MCP ramp is not required prior to entry to Fast Survey because the system is already at operating voltage following Operating Point Calibration.

Operating Point Calibration The detection system operating point calibration activity determines the balance between signal loss and crosstalk between pixels and ideally minimizes both of these values. Nominally, the discriminator threshold will be kept constant and the MCP voltage will be updated periodically to retain the desired gains and signal loss/crosstalk balance.

Ensemble Calibration Changes in the high voltage stepper outputs over time can shift sampling phase space to locations different from nominal. Such offsets are of particular relevance at the extreme end of each range (high range, low range) of the stepper supplies, where they are the largest, relative to the nominal output voltages. ESA stepper offsets are determined from the ensemble calibration activity.

HV Monitor Check Because the HV stepper voltage is not part of the regular HK packet, an activity called $H V$ monitor check confirms that the power supply can achieve the maximum required voltages and monitors the current to detect any failures in HV standoff ability.

MCP Ramp The MCP ramp activity steps the MCP voltage up to full operating voltage over the course of several minutes. During this ramp, counts are collected with an optics dark stepper configuration that eliminates incoming plasma from the aperture. These collected counts then serve as a background noise measurement that, if consistent and significant, can be subtracted from collected science data.

Burst Data Calibration Burst data calibration is performed by acquiring burst resolution data for a period of up to three spins (60 s) at a selected point within the ROI of each orbit. This data is used to perform statistical analyses, exposing and quantifying certain calibration errors. The errors are then corrected during ground processing. The particular data to be used are selected by the FPI team and marked for download through a similar interface to that used by the Scientist in the Loop.

Burst Voltage Calibration The burst voltage calibration is used may be used conjunction with ensemble calibration to provide relative calibration of the stepper voltages. Several 
spins of burst data are collected and the relative position of sharp gradients and features are compared spectrometer to spectrometer in velocity space. These measurements are then compared to pull out corrective voltages to the high voltage steppers.

\section{FPI Data Processing and Products}

FPI produces scientific data in the two modes of operation called Fast Survey and Slow Survey. In Slow Survey mode, skymaps are acquired every minute for both DES and for DIS. These data have low relevance for satisfying the primary mission objectives, and they comprise less than $\sim 1 \%$ of the FPI scientific data included in spacecraft telemetry to the ground. These skymaps are the only scientific data produced by the FPI IDPU in the Slow Survey mode of operation.

Fast Survey mode is used when the spacecraft are in the science ROI. In Fast Survey mode, the FPI produces two types of skymaps for both the DES and the DIS. Burst skymaps are acquired every $30 \mathrm{~ms}$ for the DES and every $150 \mathrm{~ms}$ for the DIS. Survey skymaps are an accumulation of burst skymaps, with counts redistributed to 32 sun-referenced angular sectors using the information on rotation phase recorded for each burst skymap. Survey skymaps are produced nominally every $4.5 \mathrm{~s}$. Each is a combination of 150 burst skymaps for the DES, or 30 burst skymaps for the DIS.

All skymaps are compressed by the IDPU prior to transmittal to the ground. Dependent on the level of complexity of the skymaps and other qualities of the data, the compression may be either lossless, or lossy. Typical compression levels achieved for the skymaps are between a factor of 4 and 5 for Burst and Slow Survey data products and slightly larger than 16 for the Fast Survey data products. The skymaps are decompressed prior to ground processing of the data sets.

\subsection{On-Board Processing of Scientific Data}

Because of telemetry limitations and the high volume of data acquired in Fast Survey mode, even with compression, it is not possible with the available downlink capacity to send all burst skymap data to the ground. The entire set of Slow Survey and Fast Survey skymaps are telemetered, but burst skymaps are queued only for intervals identified as highest priority for scientific analysis. Downlink priority is determined by the CIDP with an automated triggering process that relies on trigger data produced by FPI and other instruments. The automated selections are reviewed and can be overridden by scientists on the ground prior to downlink.

In Fast Survey mode the FPI IDPU computes two sets of burst trigger quantities; trigger terms (TT; $30 \mathrm{~ms}$ cadence) and trigger data numbers (TDN; $10 \mathrm{~s}$ cadence). TTs are quantities computed for each burst skymap (30 ms for electrons and $150 \mathrm{~ms}$ for ions). TDNs are statistical measures that characterize the variability of the trigger terms. It is only the TDNs that are used by the CIDP to evaluate burst intervals.

Each trigger term is a summation over a single burst skymap, weighted at each energy step by a factor $E^{n / 2}$, where $\mathrm{n}$ is an integer. Due to the speed required to keep pace with burst skymap production, it is not possible to calculate onboard plasma moments calibrated to physical units. Instead, the IDPU computes simplified summations with functional dependencies that are analogous to integral velocity moments of the plasma distribution functions, called "pseudo-moments".

FPI produces three trigger terms for DES and four for DIS, as defined in Table 7. In these equations, the burst skymap arrays of counts, $C_{e}$, for electrons and $C_{i}$ for ions, are expressed 
Table 7 Definitions of the electron and ion trigger terms delivered in Fast Survey data packets

\begin{tabular}{|c|c|}
\hline Trigger term & Formula \\
\hline $\begin{array}{l}\text { Electron } \\
\text { pseudo-content }\end{array}$ & $N_{e^{-}}^{\prime}=\sum_{\varepsilon \_ \text {min }}^{\varepsilon \_\max } K_{e^{-}}\left(\varepsilon, \tau_{1}\right) \sum_{\theta=0}^{15} \sum_{\varphi=0}^{31} P(\theta, \varphi) \times C_{e^{-}}(\varepsilon, \theta, \varphi)$ \\
\hline $\begin{array}{l}\text { Electron parallel } \\
\text { pseudo-flux }\end{array}$ & $F_{\text {par } n}^{\prime}=\left|\sum_{\varepsilon_{-} \min _{\text {min }}}^{\varepsilon_{\text {max }}} K_{e^{-}}\left(\varepsilon, \tau_{2}\right) \sum_{\theta=0}^{15} \sum_{\varphi=0}^{31} P(\theta, \varphi) \times \hat{\boldsymbol{b}} \cdot \hat{\boldsymbol{p}}(\theta, \varphi) \times C_{e^{-}}(\varepsilon, \theta, \varphi)\right|$ \\
\hline $\begin{array}{l}\text { Electron directional } \\
\text { pseudo-content }\end{array}$ & $\begin{array}{l}M_{e^{-} \text {dir } n}^{\prime}=\sum_{\varepsilon \_ \text {min }}^{\varepsilon} K_{e^{-}}\left(\varepsilon, \tau_{3}\right) \sum_{\theta=0}^{15} \sum_{\varphi=0}^{31} P(\theta, \varphi) \times C_{e^{-}}(\varepsilon, \theta, \varphi) \\
\text { for } T_{2}<|\mathbf{b} \cdot \mathbf{p}|<T_{3}\end{array}$ \\
\hline Ion pseudo-content & $N_{i^{+}}^{\prime}=\sum_{\varepsilon_{-} \min ^{\max }}^{\max _{i}} K_{i}\left(\varepsilon, \tau_{4}\right) \sum_{\theta=0}^{15} \sum_{\varphi=0}^{31} P(\theta, \varphi) \times C_{i^{+}}(\varepsilon, \theta, \varphi)$ \\
\hline Ion $\mathrm{X}$ pseudo-flux & $\left|F_{i+x}^{\prime}=\sum_{\varepsilon_{-} \min _{\text {min }}}^{\varepsilon_{\text {max }}} K_{i+}\left(\varepsilon, \tau_{5}\right) \sum_{\theta=0}^{15} \sum_{\varphi=0}^{31} P(\theta, \varphi) \times p_{x}(\theta, \varphi) \times C_{i+}(\varepsilon, \theta, \varphi)\right|$ \\
\hline Ion Y pseudo-flux & $\left|F_{i^{+} y}^{\prime}=\sum_{\varepsilon_{-}{ }_{-} \min }^{\max } K_{i^{+}}\left(\varepsilon, \tau_{6}\right) \sum_{\theta=0}^{15} \sum_{\varphi=0}^{31} P(\theta, \varphi) \times p_{\boldsymbol{y}}(\theta, \varphi) \times C_{i}+(\varepsilon, \theta, \varphi)\right|$ \\
\hline Ion $\mathrm{Z}$ pseudo-flux & $\left|F_{i+{ }_{z}}^{\prime}=\sum_{\varepsilon \_ \text {min }}^{\varepsilon} K_{i+}\left(\varepsilon, \tau_{7}\right) \sum_{\theta=0}^{15} \sum_{\varphi=0}^{31} P(\theta, \varphi) \times p_{z}(\theta, \varphi) \times C_{i}+(\varepsilon, \theta, \varphi)\right|$ \\
\hline
\end{tabular}

as functions of $\epsilon, \theta$, and $\varphi$, which refer to the energy step, the polar angle, and the azimuth angle of each measurement. Energy weighting is incorporated in the $K$ factors, which are stored as tables in the IDPU. The $K$ values also include scale factors chosen to optimize the range of output values. Each type of trigger term has a unique 32-element $K$ table. A "pixel pruning map", $P(\theta, \varphi)$, holds values of 0 or 1 for each of the $16 \times 32$ angular elements of the array. The calculation does not weight samples by solid angle, and the pixel map is a mechanism that allows removal of some pixels near the poles to reduce oversampling. The unit vectors $b$ and $p$ correspond to the directions of the magnetic field and the direction of incidence of particles.

The DES trigger terms are the pseudo content, parallel pseudo flux, and directional pseudo content. Because the energy weighting is accomplished with tables, which can be changed by ground command, there is some flexibility in the computation. In practice, energy weighting is chosen such that these roughly correspond to electron density, flux, and pressure. In Fast Survey mode, the direction of the vector magnetic field is passed to the FPI IDPU every $0.125 \mathrm{~s}$ by the Fields instrumentation (Torbert et al. 2014), and that direction is used to determine the component of pseudo-flux in the direction of the field. The magnetic field direction can also be used to limit the summation over angle in the computation of the directional pseudo content to produce a trigger term that, for example, is analogous to a perpendicular pressure.

The DIS trigger terms are the pseudo content, corresponding to a density, and the three Cartesian components of pseudo flux. The pseudo flux vector is referenced to axes that are fixed to the spinning body of the spacecraft.

Each TDN is the mean, variance, or delta-of-mean computed for a $10 \mathrm{~s}$ accumulation of one of the seven trigger terms. The mean and the variance are computed according to standard statistical definitions. The delta-of-mean is defined to be the difference between the mean of the current $10 \mathrm{~s}$ accumulation period and that of the previous period. Of the 21 possible TDNs, 11 were selected during mission definition for their potential to indicate that a given spacecraft is near a site of active reconnection. The electron TDNs are the variances of each of the three electron trigger terms, the mean values of the parallel pseudo flux and the directional pseudo content, and the delta-of-mean of the pseudo content. For ions, the selected TDNs are the variances of each of the four ion trigger terms, and the delta-of-mean of the ion pseudo content. The associations between TDNs and trigger terms are illustrated in 
Table 8 Identification of electron and ion trigger data numbers

Table 9 Identification of ion trigger data numbers

Fig. 57 Elements of model FPI DXS sensor

\begin{tabular}{llcc}
\hline Electron trigger term & \multicolumn{3}{l}{ Electron trigger data number } \\
\cline { 2 - 4 } & Mean & Variance & Delta of mean \\
\hline Electron pseudo content & & $\mathrm{X}$ & $\mathrm{X}$ \\
$\begin{array}{l}\text { Electron parallel pseudo } \\
\text { flux }\end{array}$ & $\mathrm{X}$ & $\mathrm{X}$ & \\
$\begin{array}{l}\text { Electron directional pseudo } \\
\text { content }\end{array}$ & $\mathrm{X}$ & $\mathrm{X}$ & \\
\hline
\end{tabular}

\begin{tabular}{|c|c|c|c|}
\hline \multirow[t]{2}{*}{ Ion trigger term } & \multicolumn{3}{|c|}{ Ion trigger data number } \\
\hline & Mean & Variance & Delta of mean \\
\hline Ion pseudo content & & $\mathrm{X}$ & $\mathrm{X}$ \\
\hline Ion X pseudo flux & & $\mathrm{X}$ & \\
\hline Ion Y pseudo flux & & $\mathrm{X}$ & \\
\hline Ion $\mathrm{Z}$ pseudo flux & & $X$ & \\
\hline
\end{tabular}

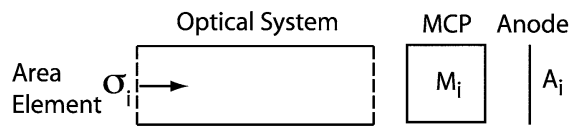

Tables 8 and 9, for DES and DIS, respectively. Note that, while the $\mathrm{x}, \mathrm{y}$, and z-components of the ion pseudo-flux are delivered to the ground in absolute value as shown in Table 7, the variance calculations are performed in the IDPU prior to performing the absolute value operation. These variance values are performed on fully signed data.

\subsection{Ground-Based Processing of Scientific Data}

The several different data sets produced on the ground share common elements of methodology. The fundamental scientific data product is the velocity distribution function, $f(\boldsymbol{x}, \boldsymbol{v}, t)$, which is directly related to the measured skymaps. It is a probability density or phase space density such that $f(\mathbf{x}, \mathbf{v}, t) d \mathbf{x} d \mathbf{v}$ is the number of particles in phase space volume element $d \mathbf{x} d \mathbf{v}$ at time $t$.

In deriving the relationship between the velocity distribution function and the skymaps, it is assumed that all of the entrance apertures of a DxS analyzer are illuminated by particles drawn from the same velocity distribution. In that case, the relationship between $f(\mathbf{x}, \mathbf{v}, t)$ at the entrance aperture $\sigma_{i}$ (Fig. 57) and the number of particles, $C_{i}^{O}$, striking section $M_{i}$ of the MCP during accumulation time $\tau$ can be expressed as follows:

$$
C_{i}^{O}=\sum_{i=0}^{15} C_{i j}^{0}
$$

where $C_{i j}^{O}$ is given by:

$$
C_{i j}^{O}=\tau \sigma_{j} \int_{v-} d^{3} v\left(\hat{\boldsymbol{n}}_{j} \cdot v\right) f(\boldsymbol{x}, \boldsymbol{v}, t) R_{i j}(\boldsymbol{v})
$$

Here, $C_{i j}^{O}$ is the number of particles striking section $M_{i}$ of the MCP when only entrance aperture $\sigma_{j}$ is illuminated, superscript " $O$ " indicates that the particles are exiting the optical 
system of Fig. 57, $\hat{\boldsymbol{n}}_{j}$ is the unit normal directed outward from the entrance aperture, $R_{i j}$ is a response matrix whose elements are measured in the laboratory by exposing each pixel $j$ to a delta function (in energy-angle space) beam and measuring the corresponding counts in pixel $i$, and the integral is taken over the region in velocity space for which $\boldsymbol{v} \cdot \hat{\boldsymbol{n}}<0$. Note that the off-diagonal elements of the $16 \times 16$ matrix $C_{i j}^{O}$ are in general non-vanishing owing to crosstalk processes occurring above the MCP (e.g., finite size of the MCP spot, scattering by the optical system, secondary electron production at the top surface of the MCP, etc.).

If the phase space density varies slowly over the region of velocity space for which $G_{i j}$ is non-vanishing, then:

$$
C_{i j}^{O} \approx \frac{2 E_{0}^{2}}{m^{2}} G_{i j} f\left(\boldsymbol{v}_{0}\right) \tau
$$

where the subscript " 0 " in $E_{0}$ and $\boldsymbol{v}_{0}$ identify the location in velocity space of the maximum of the response matrix element $R_{i j}$, and the geometric factor matrix, $G_{i j}$ has been defined:

$$
G_{i j} \equiv \frac{\sigma_{j} m^{2}}{2 E_{0}^{2}} \int_{v-} d^{3} v\left(\hat{\boldsymbol{n}}_{j} \cdot v\right) R_{i j}(\boldsymbol{v})
$$

In practice, the diagonal elements of $G_{i j}$ are just the usual geometric factors, determined by numerically integrating the energy-azimuth-angle scans measured in the laboratory. The off-diagonal elements of $G_{i j}$ are, in principle determined from polar scans by integrating the part of the polar scan centered on pixel $j$ that spreads into pixel $i$.

Downstream of the optical system in Fig. 57, particles strike the surface of the MCP and produce secondary electrons with some efficiency. There will also be some signal loss due to threshold effects which cut off a fraction of the MCP's pulse height distribution. These effects are incorporated into estimates of $G_{i j}$ as time and energy-dependent factors that are determined through a combination of laboratory and in-flight calibration.

Finally, each pre-amp has a dead time $\tau_{i}^{A M P}, \sim 100$ ns again assumed non-paralyzable:

$$
C_{i}=\frac{C_{i}^{o}}{1+C_{i}^{o} \tau_{i}^{A M P} / \tau}
$$

where $C_{i}$ is the number of counts registered by the counters of the DxS C\&DH boards during accumulation time $\tau$.

The velocity distribution function contains considerable information regarding the physical state of the plasma. Plasma fluid parameters are computed as moments of the particle velocity distributions. The technique integrates $f(\boldsymbol{v})$, weighted by powers of $\boldsymbol{v}$, over the 3 -dimensional velocity space. For example, the particle number density, $n$, is the zero-order moment, computed by multiplying the velocity distribution function by $\mathbf{v}$ to a power of zero, and integrating over velocity space. Likewise, components of the bulk flow velocity, $V_{l}$ are first-order moments, the elements of the pressure tensor, $P_{l m}$ are second-order moments, and the components of the heat-flux vector, $Q_{l}$, are third-order moments. Here, $l$ and $m$ are indices for the Cartesian components, $x, y, z$. Formulations for these 0th-3rd order moments are given as Eqs. (11)-(14).

$$
\begin{aligned}
& n=\oint d^{3} v f(\overrightarrow{\boldsymbol{v}}) \\
& \boldsymbol{V}_{l}=\frac{1}{n} \oint d^{3} v \boldsymbol{v}_{l} f(\overrightarrow{\boldsymbol{v}}) \\
& P_{l m}=m \oint d^{3} v f(\overrightarrow{\boldsymbol{v}})\left(v_{l}-V_{l}\right)\left(v_{m}-V_{m}\right) \\
& Q_{l}=\frac{m}{2} \oint d^{3} v f(\overrightarrow{\boldsymbol{v}})\left(v_{l}-V_{l}\right)(\overrightarrow{\boldsymbol{v}}-\overrightarrow{\boldsymbol{V}})^{2}
\end{aligned}
$$


Table 10 MMS FPI data sets

${ }^{1}$ After telemetry receipt at SDC

\begin{tabular}{lllll}
\hline Data set & Modes & Data types & Schedule $^{1}$ & Quality \\
\hline SITL & FS & Survey & 1.5 hour & Provisional \\
Quick look & FS, slow & Survey, burst & 24 hours & Provisional \\
Level 2 & FS, slow & Survey, burst & 30 days & Research \\
\hline
\end{tabular}

For the FPI data products, the integrals are evaluated numerically. This method has the advantage that it does not depend on a-priori assumptions about the form of the distribution functions. Corrections to the energies, and hence the velocities, due to a non-zero spacecraft surface potential are made prior to the integration. For research-quality data the measured spacecraft potential provided by the Fields Investigation (Torbert et al. 2014) is used. Prior to integration over velocity, a substitution of variable is employed to remap the integral from the range $[0, \infty]$ to the range $[0,1]$, and the trapezoidal rule is used to extrapolate the distribution function to zero energy and to the high-energy range above the top energy step of the FPI analyzers. The FPI does not discriminate ions according to mass. In computing ion distribution functions from DIS data, all ions are assumed to be protons. Measurements from the HPCA instrument (Young et al., this issue), which measures ions as a function of mass-per-charge, but at lower temporal resolution than FPI, can be used to identify intervals where this assumption is invalid.

\subsection{FPI Data Products}

The FPI data sets are produced according to definitions of product levels and the schedule for production defined in the MMS Project Data Management Plan. The data sets are distinguished according to their intended use, which determines the nature of the parameters included in each data set and the associated requirements for timeliness of production, accuracy, and level of access. Several key characteristics of the different data sets, as they apply for the FPI data, are summarized in Table 10.

The Level-1, SITL, and Quick Look products are produced at the SOC using software provided by the FPI instrument team. These are suitable for preliminary assessment of characteristics of the plasmas. They are not intended for use in scientific research because they are released prior to validation by the instrument team, may require updated calibration factors, and have not been corrected for possible variation of the spacecraft potential. The Level-1 and SITL data products are available to the MMS project only. Quick Look data are made available to the public 24 hours after receipt of telemetry from the spacecraft. The SITL data products are used by the SITL system to evaluate burst data for possible selection and download from the spacecraft. The SITL products are computed as soon as Level-1 Survey data become available, and they are available to the SITL system approximately 1.5 hours after receipt of telemetry.

The first-available research-quality data are the Level-2 data products, which will be made available through the MMS Science Data Center within 30 days. The Level-2 data are inspected by the instrument team for the presence of artifacts, and this data set incorporates any calibration changes determined in the $\sim 30$ days after receipt of data from the spacecraft. Level-2 data also utilize spacecraft potential measurements that have been validated by the Fields team. The spacecraft potential measured by Fields is used to determine particle energies as would be observed in the absence of spacecraft charging, and to eliminate the contributions of photoelectrons and secondary electrons emitted from the surface of the 
spacecraft prior to computation of fluid parameters characterizing the ambient plasma environment. In addition to those data sets addressed above, the MMS project will produce Level-3 data sets for a limited number of events. The Level-3 data products are intended to be mission-level data products that combine measurements from different MMS spacecraft, and/or from different MMS investigations. Ion fluid moments corrected for the presence of heavy ions using measurements from the HPCA are an example of a possible Level-3 data product.

\subsubsection{Descriptions of the Data Sets}

As indicated in Table 10 all data sets, with the exception of the SITL data, are produced for both the Fast Survey mode of operation and the Slow Survey mode. In the Fast Survey mode, two types of science data are produced by the CIDP. The first type of science data, termed Survey data, is routinely relayed with telemetry to the ground. The second type of science data, called Burst data, is sent to the ground only for selected intervals, due to the high sample rates and telemetry constraints. As described above, the FPI Survey data produced by the IDPU in Fast Survey mode include 4.5 s survey skymaps, Trigger Terms, and Trigger Data Numbers. The FPI Burst data produced by the IDPU in Fast Survey mode comprise burst skymaps at cadences of $30 \mathrm{~ms}$ for DES and $150 \mathrm{~ms}$ for DIS. The Slow Survey mode produces slow skymaps only. Several parameters required for interpretation of data are also included in the data sets.

FPI SITL Data Product Description The parameters provided by FPI for use by the SITL include trigger terms and trigger data numbers, plasma moments, several plasma parameters derived from the plasma moments, and arrays of counts that have been partially summed over directions or energies. These latter quantities are the basis for displays of electron pitch-angle distributions and ion energy spectra. Trigger Terms are semi-log compressed to an un-signed 8 bit value by the IDPU prior to transmission to the CIDP, and therefore are not fully representative of the terms used by the IDPU to compute TDN's. Plasma moments are computed using provisional calibration factors.

FPI Quick Look Data Description The Quick Look data are produced for both Burst and Survey data, and are made available to the scientific community and the public by the MMS Science Data Center. This data set includes provisional Level-2 moments, ion energy spectra, and electron pitch-angle distributions. Descriptions for these are the same as those given above for the SITL data.

FPI Level-2 Data Description The Level-2 data are the basic research data provided for use by MMS instrument teams and the scientific community. They are released 30 days after receipt of data from the spacecraft. They are produced for both the Burst and Survey data. They incorporate updates to calibration factors developed during that 30 day interval. The basic scientific quantities are the phase space densities and the plasma moments. Additionally, uncertainties in these quantities are provided at the one-sigma level. These uncertainties are based entirely on Poisson statistics, i.e. the counting statistics (Gershman et al. 2015a, 2015b).

The Level-2 data product also provides information suitable for producing visualizations of the 3D skymaps in the forms of: (1) Energy-time ion spectrograms (ion flux versus time) for a set of 6 directions (sunward, antisunward, dusk, dawn, and ecliptic north and south). 
(2) Electron pitch angle spectrograms (electron flux versus time) that subsume energy information by summing or averaging over several specific sets of energies. (3) Energy-time spectrograms for electrons sorted into parallel, anti-parallel, and perpendicular angular ranges to differentiate regions of differing magnetic topology for the purpose of identifying magnetic field aligned structures.

\section{Summary}

The MMS Fast Plasma Investigation measures electron and ion differential directional flux distributions on MMS at high temporal resolution. Our approach to conducting these measurements is to use for each species, electrons and ions, eight $180^{\circ}$ top hat spectrometers, packaged as dual spectrometers that we refer to as DES for electrons and DIS for ions. The two sets of four spectrometers on each spacecraft are supported by a single block redundant IDPU that communicates with the Instrument Suite CIDP, controls the spectrometers, and collects data from them.

The spectrometers were designed and built with high precision and uniformity, as verified by instrument test and calibration. Extensive ground calibration gave a solid base line for understanding the behavior of the sensors. An extensive program of in-flight calibration is executed for the purpose of maintaining a high standard of instrument calibration for all units. Quantities of interest in this calibration program include the detector systems' operating points, detection efficiencies, and HV stepping supply performance.

Table 11 provides information on mass, power, and data rates for the major components of FPI. Figure 58 shows the three primary FPI elements mounted for flight on an MMS observatory. Figure 58a shows a DES (left) and DIS (right), with solar panel cells visible in the background. The spectrometers are shown hanging off the bottom side of the instrument deck. In this flight photo, the spacecraft $\mathrm{z}$-axis points upward and, as the spacecraft spins, the two spectrometers would spin past the viewer from left to right. Figure 58b shows a flight IDPU also mounted for flight on an MMS observatory. Like the spectrometers, the IDPU hangs upside down from the instrument deck, though internal to the spacecraft with no view to the external plasma.

This approach of deploying many instruments to perform the FPI measurements required an unusually large effort in the manufacture, test, calibration, and documentation of flight hardware, which comprises 36 fully functional flight boxes (64 top hat plasma spectrometers packaged in pairs and 4 IDPUs). Innovation was required and important lessons were

Table 11 FPI mass, power, and data rate

\begin{tabular}{|c|c|c|c|c|c|c|c|}
\hline & $\begin{array}{l}\text { Avg mass } \\
{[\mathrm{kg}]}\end{array}$ & $\begin{array}{l}\text { Avg } \\
\text { SS power } \\
\text { BOL [W] }\end{array}$ & $\begin{array}{l}\text { Avg } \\
\text { FS power } \\
\text { BOL [W] }\end{array}$ & $\begin{array}{l}\text { Estimated } \\
\text { SS power } \\
\text { EOL }[W]\end{array}$ & $\begin{array}{l}\text { Estimated } \\
\text { FS power } \\
\text { EOL }[\mathrm{W}]\end{array}$ & $\begin{array}{l}\text { Data SS } \\
\text { (bps) }\end{array}$ & $\begin{array}{l}\text { Data FS } \\
\text { (bps) }\end{array}$ \\
\hline $1 \mathrm{DES}$ & 5.87 & 5.5 & 5.8 & 8.3 & 8.7 & 512 & 1195869 \\
\hline 1 DIS & 6.21 & 5.2 & 5.2 & 6.9 & 6.9 & 512 & 385017 \\
\hline $1 \mathrm{IDPU}$ & 6.19 & 4.8 & 5.2 & 4.9 & 5.3 & 474 & 500 \\
\hline $\begin{array}{l}\text { Full FPI } \\
\text { (4 DES, } \\
4 \text { DIS, } \\
1 \text { IDPU) }\end{array}$ & 54.51 & 47.6 & 49.1 & 65.5 & 67.6 & 4570 & 6324042 \\
\hline
\end{tabular}

$\mathrm{SS}=$ Slow Survey, FS = Fast Survey 
Fig. 58 Elements of the FPI, installed and ready for flight
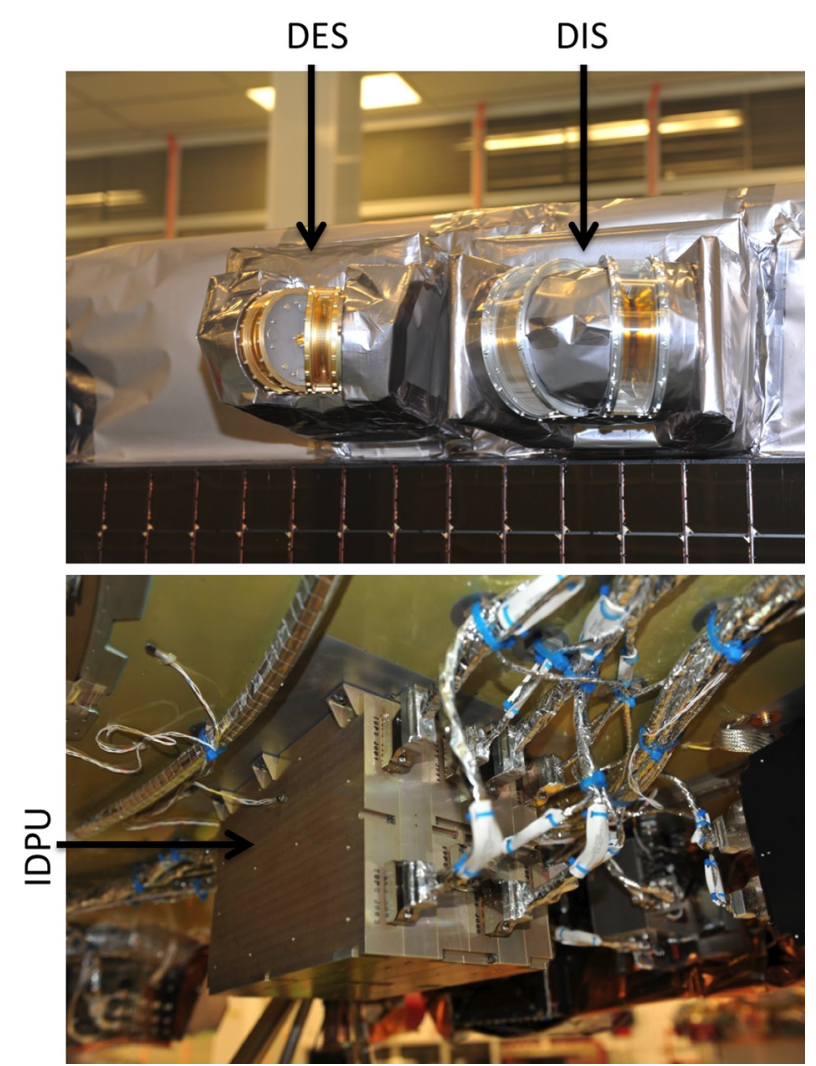

learned. FPI's high time resolution and quality sensor designs and their characterizations will enable new discoveries exploring Earth's magnetospheric electron and ion phase space distributions in and near the diffusion regions of reconnecting plasmas.

Open Access This article is distributed under the terms of the Creative Commons Attribution 4.0 International License (http://creativecommons.org/licenses/by/4.0/), which permits unrestricted use, distribution, and reproduction in any medium, provided you give appropriate credit to the original author(s) and the source, provide a link to the Creative Commons license, and indicate if changes were made.

\section{References}

A.P. Biddle, J.M. Reynolds, Integrated development facility for the calibration of low-energy charged particle flight instrumentation. Rev. Sci. Instrum. 57, 572 (1985). doi:10.1063/1.1138873

F. Bordoni, Channel electron multiplier efficiency for 10-1000 eV electrons. Nucl. Instrum. Methods 97, 405 (1971). doi:10.1016/0029-554X(71)90300-4

J.L. Burch, R. Goldstein, T.E. Cravens, W.C. Gibson, R.N. Lundin, C.J. Pollock, J.D. Winningham, D.T. Young, RPC-IES: the ion and electron sensor of the Rosetta Plasma Consortium. Space Sci. Rev. 128, 697-712 (2007). doi:10.1007/s11214-006-9002-4

C.W. Carlson, D.W. Curtis, G. Paschmann, W. Michael, An instrument for rapidly measuring plasma distribution functions with high resolution. Adv. Space Res. 2, 7 (1982). doi:10.1016/0273-1177(82)90151-X

C.W. Carlson, J.P. McFadden, P. Turin, D.W. Curtis, A. Magoncelli, The electron and ion plasma experiment for FAST. Space Sci. Rev. 98, 33-66 (2001). doi:10.1023/A:1013139910140

G.A. Collinson, The computer simulated design of an improved plasma analyser towards an electron spectrometer for Solar Orbiter. Ph.D. Thesis, University College London (2010) 
G.A. Collinson, D.O. Kataria, A.J. Coates, S.M.E. Tsang, C.S. Arridge, G.R. Lewis, R.A. Frahm, D.J. Winningham, S. Barabash, Electron optical study of the Venus Express ASPREA-4 Electron Spectrometer (ELS) top-hat electrostatic analyser. Meas. Sci. Technol. 20, 055204 (2009)

G.A. Collinson et al., The geometric factor of electrostatic plasma analyzers: a case study from the Fast Plasma Investigation for the Magnetospheric Multiscale mission. Rev. Sci. Instrum. 83, 033303 (2012). doi:10.1063/1.3687021

W. Daughton, J. Scudder, H. Karimabadi, Fully kinetic simulations of undriven magnetic reconnection with open boundary conditions. Phys. Plasmas 13, 072101 (2006). doi:10.1063/1.2218817

D. Delcourt et al., The mass spectrum analyzer (MSA) onboard BEPI COLOMBO MMO: scientific objectives and prototype results. Adv. Space Res. 43, 869-874 (2009). doi:10.1016/j.asr.2008.12.002

H.O. Funsten, R. Harper, D. McComas, Absolute detection efficiency of space-based ion mass spectrometers and neutral atom imagers. Rev. Sci. Instrum. 76, 053301 (2005). doi:10.1063/1.1889465

K. Furuya, Y. Hatano, Pulse-height distribution of output signals in positive ion detection by a microchannel plate. Int. J. Mass Spectrom. 218, 237-243 (2002). doi:10.1016/S1387-3806(02)00725-X

D.J. Gershman, J.C. Dorelli, A.F. Viñas, C.J. Pollock, The calculation of moment uncertainties from velocity distribution functions with random errors. J. Geophys. Res. Space Phys. 120, 6633-6645 (2015a). doi:10.1002/2014JA020775

D.J. Gershman et al., The parameterization of top-hat particle sensors with microchannel-plate-based detection systems and its application to the Fast Plasma Investigation on NASA's Magnetospheric Multiscale Mission. Measurement Techniques in Solar and Space Physics Meeting. NCAR Center Green Campus, Boulder, CO (2015b)

U. Gliese et al., Improved detection system description and new method for accurate calibration of microchannel plate based instruments and its use in the Fast Plasma Investigation on NASA's Magnetospheric Multiscale Mission. Measurement Techniques in Solar and Space Physics Meeting. NCAR Center Green Campus, Boulder, CO (2015)

D.J. McComas et al., The Jovian Auroral Distributions Experiment (JADE) on the Juno Mission to Jupiter. Space Sci. Rev. 1-97 (2013). doi:10.1007/s11214-013-9990-9

J. Oberheide, P. Wilhelms, M. Zimmer, New results on the absolute ion detection efficiencies of a microchannel plate. Meas. Sci. Technol. 8, 351-354 (1997). doi:10.1088/0957-0233/8/4/001

H. Rème et al., The Cluster Ion Spectrometry (CIS) experiment. Space Sci. Rev. 79, 303-350 (1997). doi:10.1023/A:1004929816409

Y. Saito et al., In-flight performance and initial results of Plasma Energy Angle and Composition Experiment (PACE) on SELENE (Kaguya). Space Sci. Rev. 154, 265-303 (2010). doi:10.1007/s11214-010-9647-x

J.-A. Sauvaud et al., The INTERBALL-Tail ELECTRON experiment: initial results on the low-latitude boundary layer of the dawn magnetosphere. Ann. Geophys. 15, 587-595 (1997). doi:10.1007/s00585997-0587-z

J.-A. Sauvaud et al., The IMPACT Solar Wind Electron Analyzer (SWEA). Space Sci. Rev. 136, 227-239 (2007). doi:10.1007/978-0-387-09649-0_9

J. Scudder et al., Hydra-a 3-dimensional electron and ion hot plasma instrument for the POLAR spacecraft of the GGS mission. Space Sci. Rev. 71, 459-495 (1995). doi:10.1007/BF00751338

R. Torbert et al., The FIELDS instrument suite on MMS: scientific objectives, measurements, and data products. Space Sci. Rev. (2014). doi:10.1007/s11214-014-0109-8

P.W. Valek, Development of spacecraft borne instrumentation and analysis of low energy ionospheric outflow. Ph.D. Thesis. Auburn University, Source DAI-B 62/06 (2001)

S. Yokota, Y. Saito, K. Asamura, T. Mukai, Development of an ion energy mass spectrometer for application on board three-axis stabilized spacecraft. Rev. Sci. Instrum. 76, 014501 (2005). doi:10.1063/1.1834697

D.T. Young et al., $2 \pi$-radian field-of-view toroidal electrostatic analyzer. Rev. Sci. Instrum. 59, 743 (1988). doi:10.1063/1.113982

D.T. Young et al., Plasma Experiment for Planetary Exploration (PEPE). Space Sci. Rev. 129, 327-357 (2007). doi:10.1007/s11214-007-9177-3

D.T. Young et al., Hot plasma composition analyzer for the magnetospheric multiscale mission. Space Sci. Rev. (2014). doi:10.1007/s11214-014-0049-6 